Aus der Poliklinik für Zahnärztliche Prothetik

(Prof. Dr. med. dent. R. Bürgers)

im Zentrum Zahn-, Mund- und Kieferheilkunde

der Medizinischen Fakultät der Universität Göttingen

\title{
Vergleichende Untersuchungen zur Unterkieferbewegung mittels Videosequenz- und 3D-Ultraschallanalyse
}

\author{
INAUGURAL - DISSERTATION \\ zur Erlangung des Doktorgrades \\ für Zahnheilkunde \\ der Medizinischen Fakultät der \\ Georg-August-Universität zu Göttingen
}

vorgelegt von

Janna Marie Edelhoff

aus

Neustadt am Rübenberge

Göttingen 2015 
Dekan:

I. Berichterstatter:

II. Berichterstatter/in:

III. Berichterstatter/in:
Prof. Dr. rer. nat. H. K. Kroemer

Priv. - Doz. Dr. med. dent. N. Gersdorff

Priv. - Doz. Dr. med. dent. S. Sennhenn-Kirchner

Prof. Dr. med. M. Schön

Tag der mündlichen Prüfung: $\quad 23.09 .2015$ 


\section{Inhaltsverzeichnis}

1. Einleitung

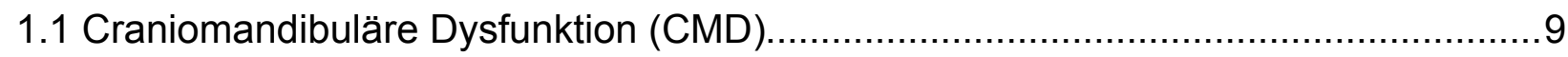

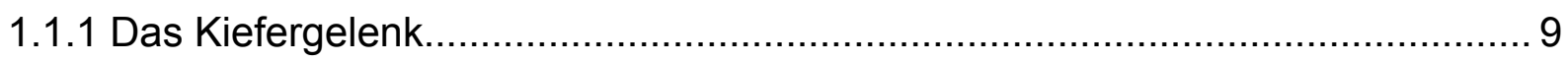

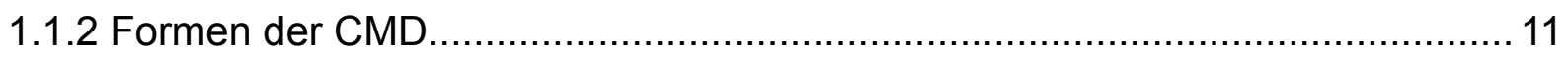

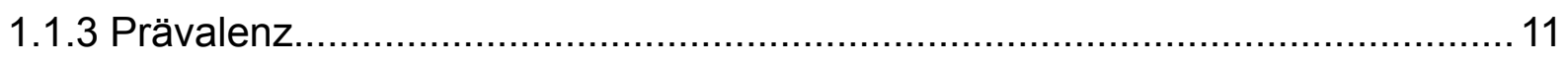

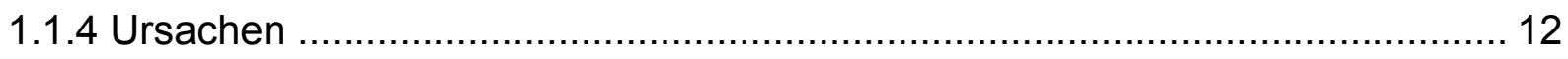

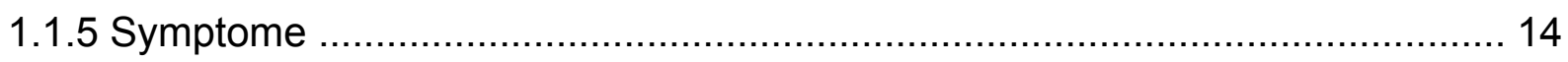

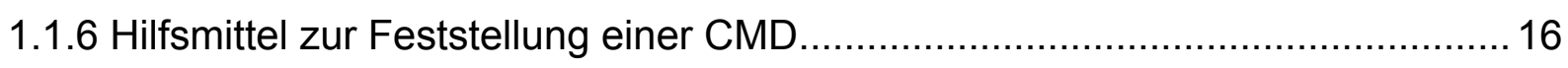

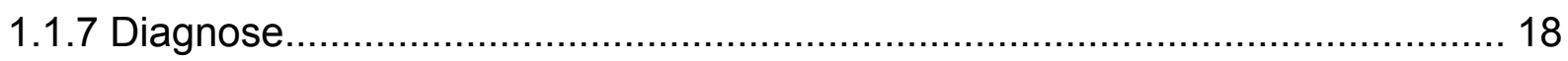

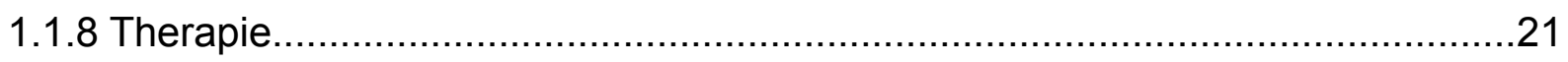

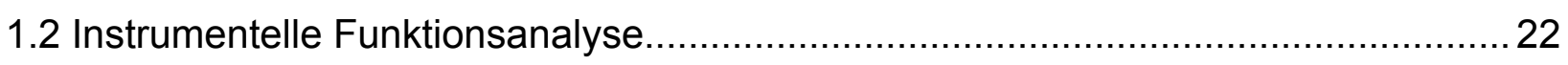

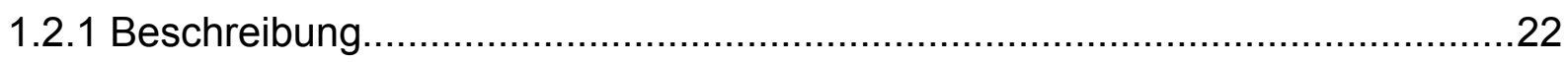

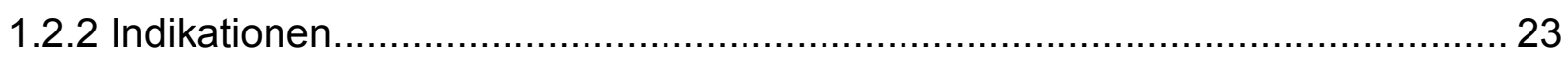

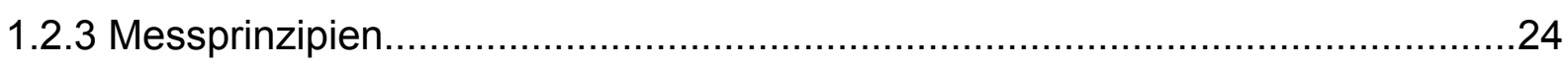

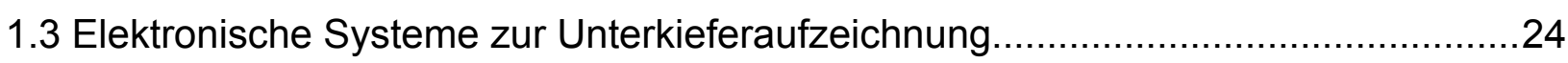

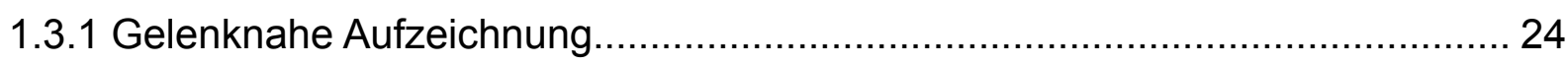

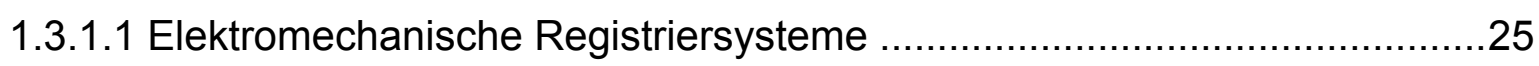

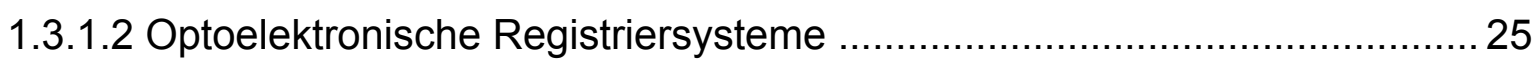

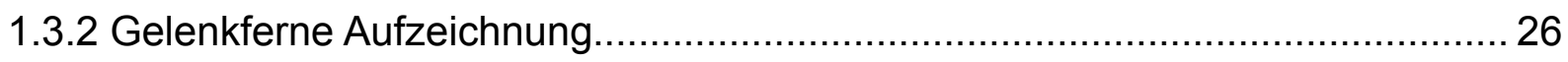

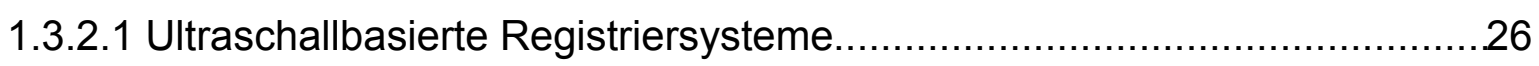

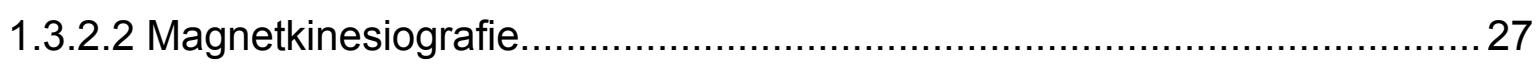

1.4 Entwicklung der elektronischen und optoelektronischen Registriersysteme..........28

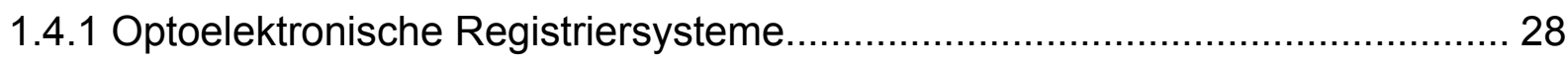

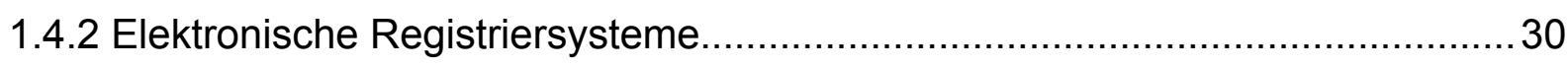

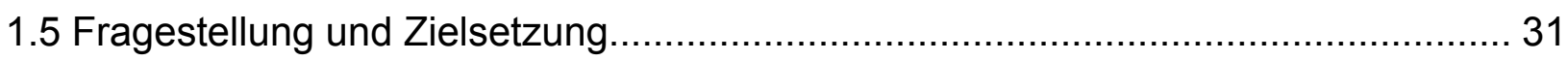




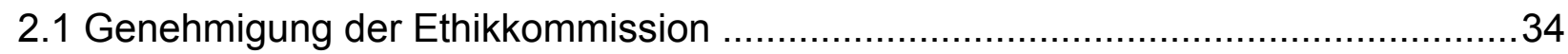

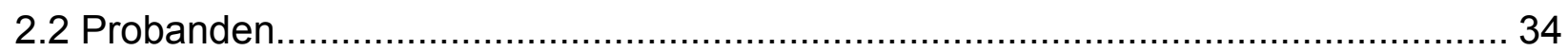

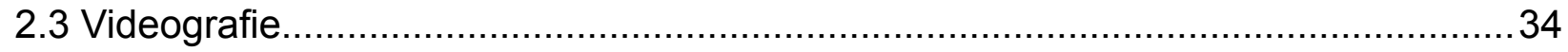

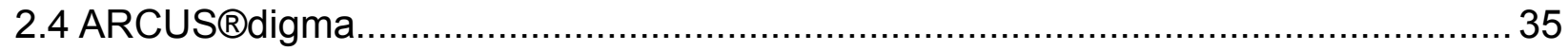

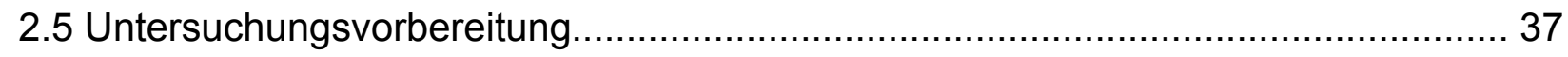

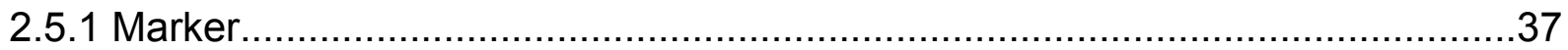

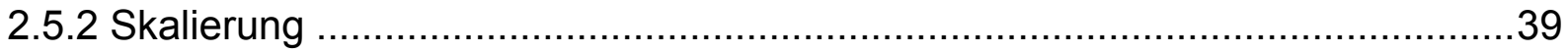

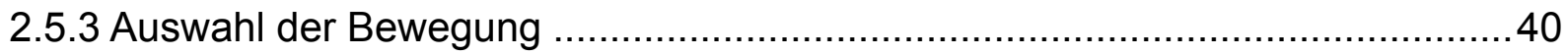

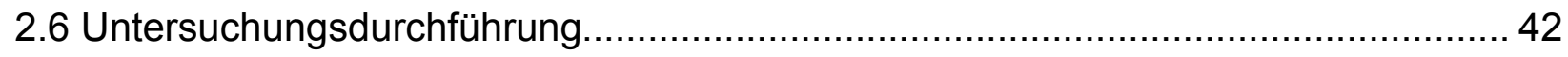

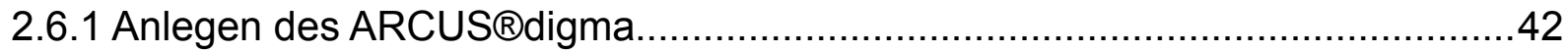

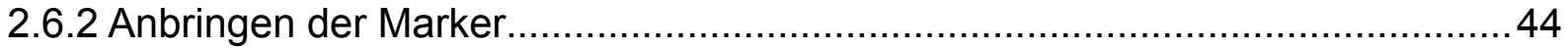

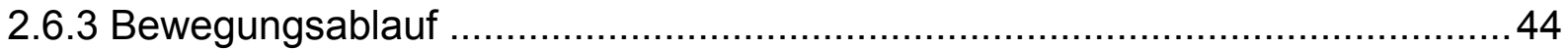

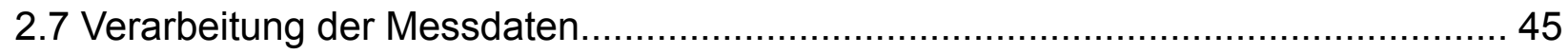

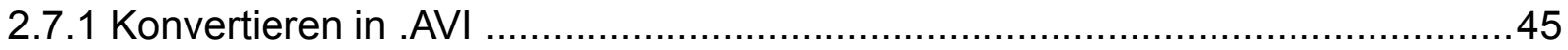

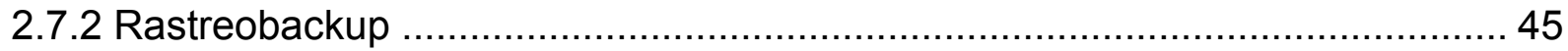

2.7.3 MATLAB® (MATrix LABoratory) .............................................................. 47

2.7.4 Export der ARCUS®digma-Daten .......................................................... 48

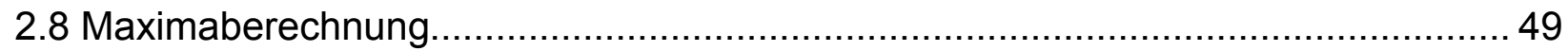

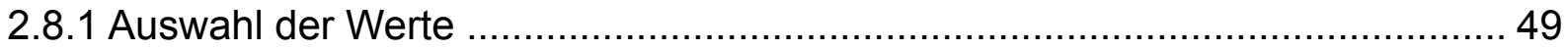

2.8.2 Maxima Videoanalyse - frontale Grenzbewegung.....................................50

2.8.3 Maxima Videoanalyse - Posselt sagittal.....................................................50

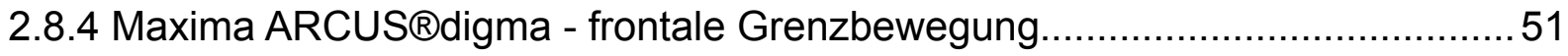

2.8.5 Maxima ARCUS $®$ digma - Posselt sagittal................................................. 52

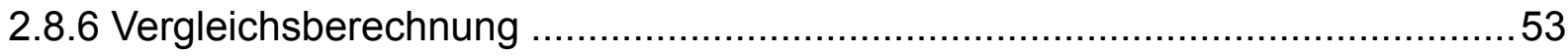

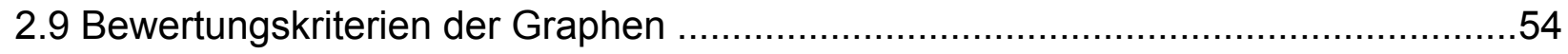

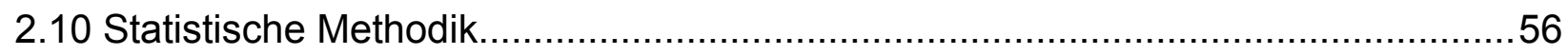




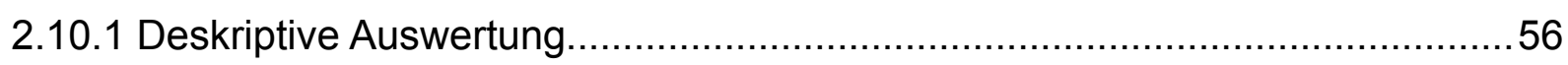

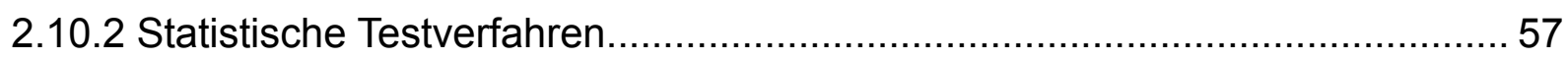

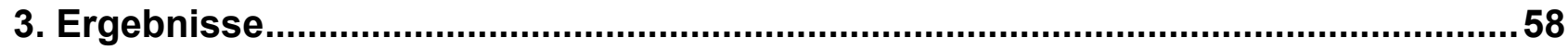

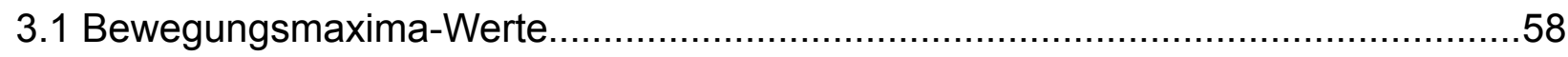

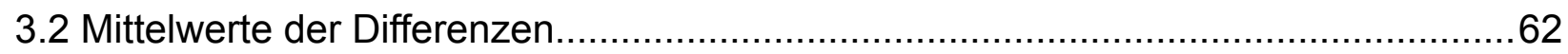

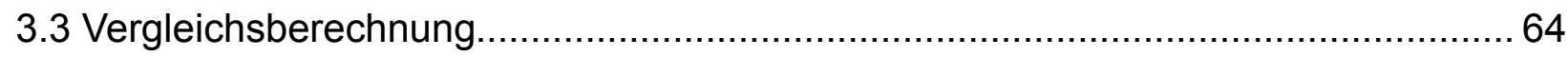

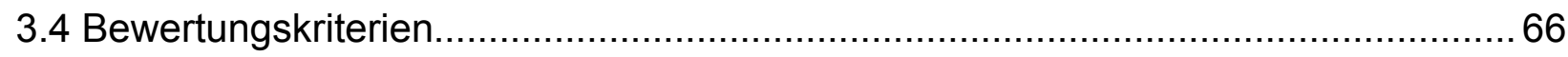

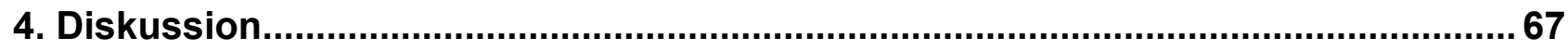

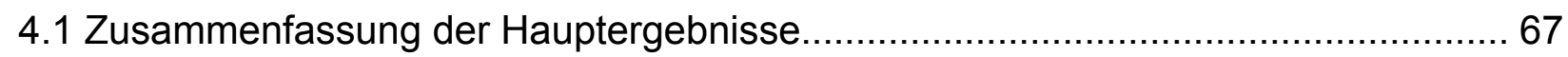

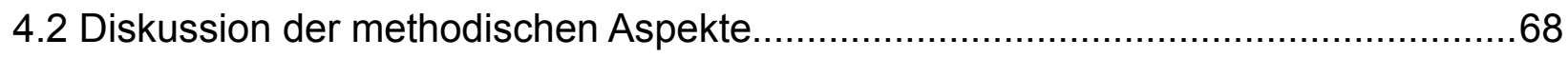

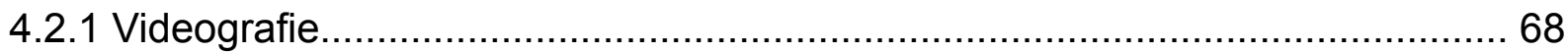

4.2.1.1 Aufnahme der Bewegungen...................................................................68

4.2.1.2 Analyse der Aufnahmen........................................................................ 70

4.2.1.3 Videoanalyse allgemein.................................................................... 73

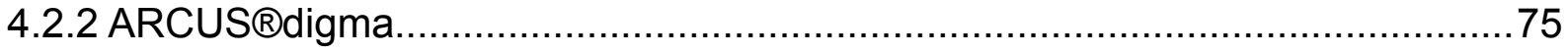

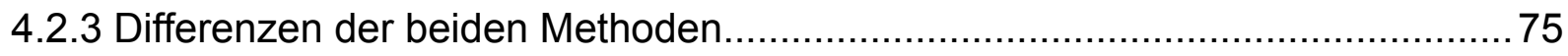

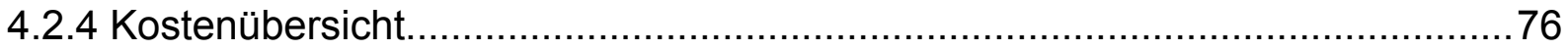

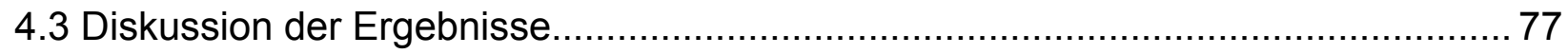

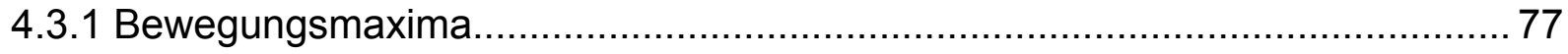

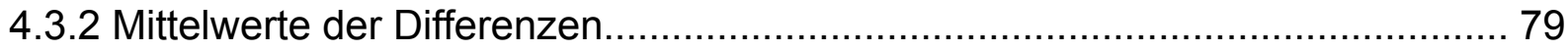

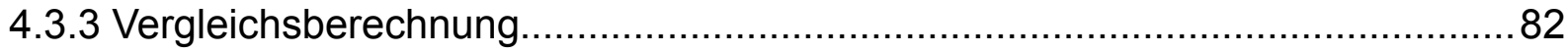

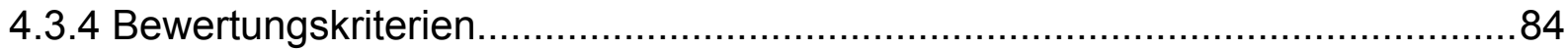

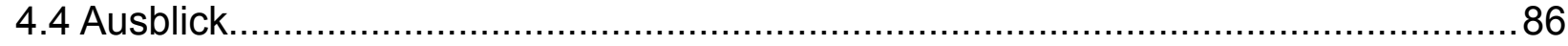

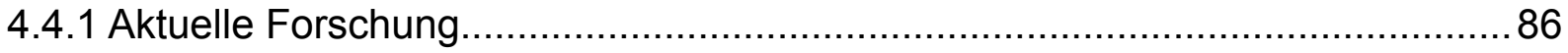

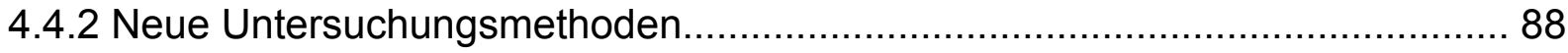

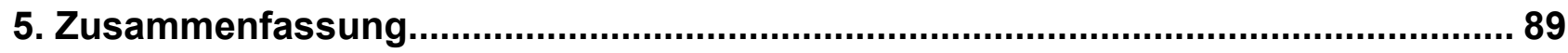




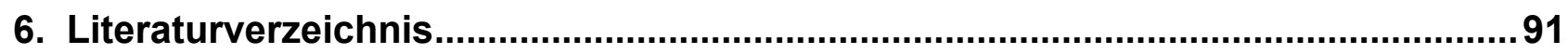

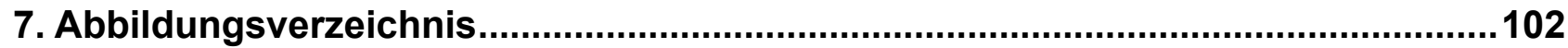

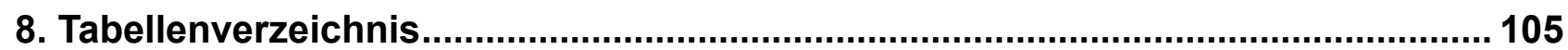

9. Anhang

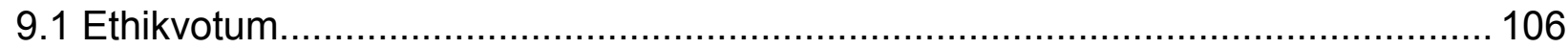

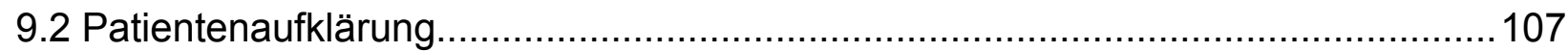

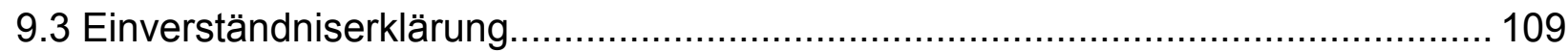

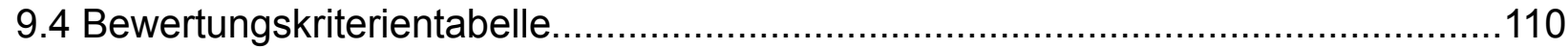




\section{Abkürzungsverzeichnis}

\begin{tabular}{|c|c|}
\hline Abb. & Abbildung \\
\hline. $\mathrm{AVI}$ & Audio Video Interleave \\
\hline AVS & Application Visualization System \\
\hline CAD/CAM & Computer-aided design/ Computer-aided manufacturing \\
\hline $\mathrm{cm}$ & Zentimeter \\
\hline CMD & Craniomandibuläre Dysfunktion \\
\hline CT & Computertomographie \\
\hline DGZMK & Deutsche Gesellschaft für Zahn-, Mund- und Kieferheilkunde \\
\hline DMS & Deutsche Mundgesundheitsstudie \\
\hline Dr. & Doktor \\
\hline $\begin{array}{l}\text { ECRS } \\
\text { et al. }\end{array}$ & $\begin{array}{l}\text { Elektronisches Computergestütztes Registriersystem } \\
\text { et alii }\end{array}$ \\
\hline GAG & Glykosaminoglykane \\
\hline ICR & Instantaneous centers of rotation \\
\hline LED & Licht-emittierende Diode(n) \\
\hline $\mathrm{m}$ & Meter \\
\hline MAP & Myoarthropatie \\
\hline MATLAB® & Matrix Laboratory \\
\hline $\mathrm{mm}$ & Millimeter \\
\hline MTS & Moving Picture Experts Group (MPEG) Transport Stream \\
\hline $\begin{array}{l}\text { Priv. - Doz. } \\
\text { rer. nat. }\end{array}$ & $\begin{array}{l}\text { Privat-Dozent } \\
\text { rerum naturalium }\end{array}$ \\
\hline $\mathrm{RDC} / \mathrm{TMD}$ & Research Diagnostic Criteria for Temporomandibular Disorders \\
\hline Tab. & Tabelle \\
\hline.$t x t$ & Textdatei \\
\hline UMG & Universitätsmedizin Göttingen \\
\hline UNER & Universidad Nacional de Entre Rios \\
\hline UTF & Unicode Transformation Format \\
\hline ZMK & Zahn-, Mund- und Kieferheilkunde \\
\hline
\end{tabular}




\section{Einleitung}

Neben den klassischen Bereichen der Zahnmedizin, wie der konservierenden Zahnheilkunde und der Prothetik, sind heute die Erkrankungen des Kauapparates fester Bestandteil des klinischen Alltags.

Sie wurden durch verschiedene Begriffe wie Craniofacial Dysfunction (Krestan et al. 2001), Myoarthropathie (Schulte 1970, Türp et al. 2005) und Temporomandibular Disorders (Al-Saleh et al. 2012, LeResche 1997, McNeill 1997) beschrieben. Die DGZMK führte den Begriff der Craniomandibulären Dysfunktion (CMD) ein, welcher auch in dieser Studie verwendet werden soll.

Unter diesem Begriff werden multikausale Funktionsstörungen des Kausystems zusammengefasst. Man differenziert zwischen Störungen in der Okklusion, der Muskulatur, des Kiefergelenks, des Nervensystems oder einer Kombination aus diesen Faktoren (Hanel 1984). Symptome äußern sich unter anderem durch Kiefergelenkknacken und -reiben, eine eingeschränkte Mundöffnung und Muskelschmerzen. Darüber hinaus können sich Spannungen verselbstständigen und in einen Kreislauf des positiven Feedbacks münden.

Studien zeigen, dass es sich bei der CMD um eine weit verbreitete Erkrankung handelt. Die Ergebnisse gehen dabei weit auseinander. Strub berichtet von $5 \%$ Erkrankten, bei Manfredini sind es rund $10 \%$, im Bundes-Gesundheitssurvey werden für Männer $12 \%$ und für Frauen 20,4 \% angegeben und nach Siebert leidet eine Bevölkerungsgruppe von bis zu 80 \% an Funktionsstörungen (Strub et al. 2011, Manfredini et al. 2011, Kohlmann 2002, Bellach et al. 1999, Siebert 1987).

Um die Ursachen dieser Erkrankung zu ergründen, wurden verschiedene Analysemethoden entwickelt, die heute meist vereint angewendet werden. Neben dem Gespräch mit dem Patienten (Anamnese) sind das Abhören (Auskultation) und Abtasten (Palpation) von großer Bedeutung. Darüber hinaus besteht die Möglichkeit, mit Zuhilfenahme von funktionsdiagnostischen Geräten die Bewegungen des Unterkiefers aufzuzeichnen, um aus den Graphen Normabweichungen ablesen zu können.

Moderne Geräte arbeiten heute auf elektronischer, optoelektronischer, elektromagnetischer oder Ultraschall-Basis und mit einer elektronischen Datenverarbeitung (Engelhardt et al. 2002).

Die Kosten dieser Geräte sind allerdings sehr hoch und können nicht von allen klinischen 
Einrichtungen getragen werden. Nach M. Schmitter entscheiden am Ende ökonomische Faktoren, in welchem Umfang Maßnahmen zur Verbesserung der Funktionen des Zahnersatzes ergriffen werden (Schmitter et al. 2011). Auch Pinheiro bemängelt, dass die Systeme aufgrund ihrer hohen Kosten von vielen Kliniken nicht genutzt werden können. Darüber hinaus ist die Anwendung teils kompliziert und zeitaufwendig (Pinheiro et al. 2011).

In der vorliegenden Untersuchung sollte aus diesen Gründen eine neue Methode der instrumentellen Funktionsanalyse getestet werden. Diese hatte den Anspruch, eine kostengünstige Alternative zu etablierten Analysegeräten darzustellen. Darüber hinaus sollten Arbeitsaufwand und Invasivität verringert werden, ohne die Messgenauigkeit zu beeinträchtigen.

Die Messungen sollen mit einer neuen Art der Videoanalyse durchgeführt und mit den Ergebnissen eines auf Ultraschallbasis arbeitenden elektronischen Gesichtsbogens verglichen werden.

\subsection{Craniomandibuläre Dysfunktion (CMD)}

\subsubsection{Das Kiefergelenk}

Das Kiefergelenk ist das einzige Doppelgelenk im menschlichen Körper und besteht aus knöchernen und bindegewebigen Anteilen. Der Condylus mandibulae und die Fossa articularis mit dem Tuberculum articulare bilden dabei die knöchernen Gelenkkörper. Beide Strukturen sind von Faserknorpel überzogen. Zu den bindegewebigen Anteilen gehört der Discus articularis. Dieser lässt sich in drei Zonen einteilen: die anteriore, die mediale und die posteriore Zone, die auch bilaminäre Zone genannt wird. Im vorderen Teil besteht der Discus aus fibrösem Material mit eingestreuten Knorpelzellen. Dieser Teil ist mit dem Caput infratemporale des M. Pterygoideus lateralis und der Gelenkkapsel verbunden. Der mittlere Teil ist von Faserknorpel geprägt und der hintere Teil bildet die bilaminäre Zone. Diese Zone besteht in ihrem oberen Anteil aus fibroelastischem Gewebe, welches am hinteren Rand der Fossa mandibularis verankert ist. Der untere Anteil besteht aus sehr straffem und fibrösem Gewebe, welches am Hinterrand des Condylus mandibulae befestigt ist. Die bilaminäre Zone ist reich innerviert und vaskularisiert (Okeson und De Leeuw 2011). 
Durch die sensible Innervation werden schon sehr kleine Veränderungen wahrgenommen, die bei Fehlstellungen als Druck oder Schmerz empfunden werden können (Ahlers 1998). Die Gelenkkapsel (Capsula articularis) besteht aus einer dünnen bindegewebigen Schicht und wird lateral durch das Ligamentum laterale (Arcus zygomaticus - Prozessus condylaris) verstärkt. Als Führungsbänder wirken darüber hinaus das Ligamentum stylomandibulare (Prozessus styloideus - Angulus mandibulae) sowie das Ligamentum sphenomandibulare (Spina ossis sphenoidalis - Lingua mandibulae). Diese beiden Bänder stehen aber nicht im direkten Zusammenhang mit der Gelenkkapsel.

Wie schon erwähnt, ist das menschliche Kiefergelenk ein Doppelgelenk. Darüber hinaus wird es durch den Discus articularis in einen diskotemporalen und einen diskomandibulären Teil untergliedert. Dadurch stellen das rechte sowie das linke Kiefergelenk funktionell betrachtet jeweils eine Kombination zweier Gelenke in sich dar. Bei der Mundöffnung vollführt das untere Teilgelenk eine Drehbewegung und das obere zur selben Zeit eine Gleitbewegung (Platzer 2009). Die Mundöffnung besteht somit aus einer kombinierten Drehund Gleitbewegung. Dabei gleitet der Discus articularis zusammen mit dem Kondylus aus der Fossa auf das Tuberculum articulare in ventraler und kaudaler Richtung (Lehmann et al. 2009).

Die Bewegungen des Kiefergelenks sind sehr komplex, da das Gelenk über drei Bewegungsachsen verfügt, die neben Rotations- auch Translationsbewegungen zulassen. Die Translationsbewegung folgt dabei nicht einer Geraden, sondern hat einen gekrümmten Verlauf (Schierz und Reißmann 2008). Darüber hinaus müssen die Bewegungen im rechten und linken Kiefergelenk nicht übereinstimmen. Bei der Laterotrusion bewegt sich der Kondylus der Mediotrusionsseite nach ventral und medial, der Kondylus der Laterotrusionsseite wird hingegen als „ruhender Kondylus“ bezeichnet und stellt das Bewegungszentrum dar (Lehmann et al. 2009). Durch die dreidimensionale Rotations- und Translationsbewegung rotiert die Mandibula ständig um eine neue Achse. Dies wurde schon 1914 von Thouren beschrieben (Thouren 1914). Durch die Veränderung der Rotationsachse besteht keine klare räumliche Zuordnung des Unterkiefers zur Schädelbasis, was sowohl die Bewegungsanalyse als auch Diagnose und Therapie von Kiefergelenkerkrankungen erschwert. 


\subsubsection{Formen der CMD}

Ein häufiger Befund in der Zahnheilkunde ist die CMD (Gonçalves et al. 2010). Diese Erkrankung beschreibt eine Funktionsstörung im stomatognathen System. Die schmerzhaften Funktionsstörungen werden auch Myoarthropathien (MAP) genannt. Diese Erkrankung beschreibt eine Untergruppe der CMD. Der Begriff wurde 1970 von Schulte (Tübingen) eingeführt und setzt subjektive Störungsempfindungen oder Beschwerden voraus. Das Leiden beziehungsweise der Schmerz (griechisch pathós) stehen hier im Vordergrund. Die MAP wird deshalb auch als Schmerzdysfunktionssyndrom beschrieben (Strub et al. 2011). Eine CMD kann in kompensierter sowie dekompensierter Form vorliegen (Kralijevic et al. 2003). Bei der kompensierten Form geht der Körper durch Regulations-, Reparatur- und Umbauprozesse gegen die Symptome der Erkrankung vor. Sobald diese Gegenregulation versagt, spricht man von einer dekompensierten CMD. Auslöser für die Dekompensation können unter anderem konservierende sowie prothetische Versorgungen sein.

\subsubsection{Prävalenz}

Die CMD tritt am häufigsten bei Personen im Alter zwischen 20 und 40 Jahren auf (Carlsson 1999; Ingawalé und Goswami 2012). Das Ergebnis der Dritten Deutschen Mundgesundheitsstudie (DMS-III) aus dem Jahr 1997 zeigt, dass in der Altersgruppe der 35- bis 44-Jährigen etwa $5 \%$ der Befragten an MAP-Symptomen litten (Strub et al. 2011). Behandlungsbedürftigkeit besteht nach Türp bei etwa $3 \%$ (Türp 2003). Nach Manfredini leiden 6 - 12 \% der allgemeinen Bevölkerung an myofazialem Schmerz und 8,9 - 15,8 \% an einer Diskusverlagerung mit Reposition (Manfredini et al. 2011).

Die objektiven und subjektiven Einschätzungen der Erkrankung gehen dabei oft auseinander. In einer Studie wurde festgestellt, dass bei 60 - 80 \% der Untersuchten Funktionsstörungen vorlagen, aber nur $15 \%$ davon waren auch subjektiv krank (Siebert 1987).

Im deutschen Bundes-Gesundheitssurvey 1998 ( $n=7124$ Erwachsene) gaben 4,7 \% bzw. 12,0 \% der Männer und 8,6 \% bzw. 20,4 \% der Frauen an, in den letzten sieben Tagen bzw. 12 Monaten an Schmerzen im Bereich des Gesichts, der Kaumuskulatur, der Kiefergelenke oder der Ohren gelitten zu haben (Bellach et al. 1999, Kohlmann 2002). In einer brasilianischen Studie mit 100 Probanden waren von den an CMD Erkrankten 88 \% Frau- 
en und nur $12 \%$ Männer (Costa et al. 2012).

Die Ergebnisse der Studien weisen auf die Wahrscheinlichkeit hin, dass Frauen häufiger an MAP-Symptomen (Schmerzen) leiden als Männer (Carlsson 1999; Ingawalé und Goswami 2012). Aus diesem Grund werden Frauen auch häufiger als Männer wegen einer CMD-Erkrankung behandelt.

Weshalb Funktionsstörungen beim weiblichen Geschlecht stärker vertreten sind, steht nicht fest. Es könnten weibliche Sexualhormone an der Schmerzübertragung beteiligt sein, was dazu führt, dass Frauen die Schmerzen im Kiefergelenk stärker empfinden und so eine Behandlung häufiger in Anspruch nehmen (Detamore und Alhanasiou 2003).

\subsubsection{Ursachen}

Die CMD ist eine multifaktorielle Erkrankung. Neben dem Erbgut und Umwelteinflüssen (Trauma, Zahnbehandlungen) spielen auch psychosoziale Elemente als Ursachen eine Rolle.

Dysgnathien: Ein Grund für das Auftreten einer CMD können nicht therapierte Dysgnathien sein. Es kommt hierbei zur unphysiologischen Okklusion, die sich klinisch in einem Zwangbiss, einer Nonokklusion oder Störkontakten äußern kann. Dieser Zustand kann iatrogen durch prothetische Versorgungen und kieferorthopädische Maßnahmen verursacht sein, die eine physiologische Interkuspidation (Ineinandergreifen der Zähne) verhindern. Die Fehlokklusion kann jedoch auch einen genetischen Ursprung haben oder durch Angewohnheiten (Habits) erworben sein. Darüber hinaus kann der Verlust einer ausreichenden Abstützung im Seitenzahnbereich zu einer verminderten Vertikaldimension mit Umbauprozessen und Beschwerden im Kiefergelenk führen (Costa et al. 2012).

Okklusionsprobleme müssen aber nicht immer Auslöser einer CMD sein. Sie können selbst durch z.B. Muskelverspannungen verursacht sein, die der eigentliche Ursprung der Erkrankung sind (Okeson und De Leeuw 2011).

Diskusverlagerungen: Der Störung des Kausystems kann ebenfalls eine Diskusverlagerung zugrunde liegen. Hierbei liegt der Discus articularis nicht mehr auf dem Kondylus, 
sondern in anteriorer oder lateraler Position. Diese Verlagerung kann partiell oder total sein. Darüber hinaus kann der Diskus entweder in der veränderten Position verbleiben (ohne Reposition) oder während der Bewegung auf den Kondylus zurückspringen (mit Reposition). Die Reposition findet in der Regel in der initialen, der intermediären oder der terminalen Phase der Mundöffnung statt (Ahlers 1998).

Schmerzen: Eine Funktionsstörung kann des Weiteren durch Schmerzen verursacht werden. Eine Pulpitis, eine Osteoarthritis oder rheumatologische Arthritis sowie ein Gelenktrauma können den Patienten in eine Schonhaltung oder Schonbewegung zwingen, um den Schmerzen auszuweichen (Ingawalé und Goswami 2012). Dies kann zu Fehlfunktionen der Muskulatur und zu Muskelverspannungen führen, die eine CMD verursachen (Mumford 1989).

Stress: Eine der häufigsten Ursachen für die CMD-Erkrankung ist der Faktor Stress. Er kann physischer (Schlafmangel, Lärm), psychischer (Angst, Überforderung) und sozialer (Mobbing, mangelnder Kontakt) Natur sein (Strub et al. 2011). Stress kann die Aktivität der Kaumuskulatur erhöhen und in einen Dauermodus versetzen. Die Aktivitätssteigerung äußert sich häufig durch nächtliches Knirschen und Pressen. Diese Tätigkeit wird auch „Bruxieren“ genannt. Bruxismus führt zum verstärkten Einsatz der Muskulatur und als Folge zur vermehrten Beanspruchung von dentalen, parodontalen, ossären und arthrogenen Strukturen, die in einer Überlastung des Kausystems münden können (Ahlers 1998; Strub et al. 2011).

Traumata: Neben diesen Faktoren können auch Traumata Ursache für eine CMD sein. Man unterscheidet zwischen irreversiblen Makrotraumen, die sich in einer Diskusausdünnung, Diskusdeformation oder -perforation durch lang anhaltende Fehlbelastungen äußern und reversiblen Mikrotraumen, die durch raue Diskusoberflächen, Ischämie oder Hypoxie gekennzeichnet sind (Mumford 1989; Okeson und De Leeuw 2011). Die bestehende Fehlbelastung kann durch die Strukturveränderung des Diskus noch verstärkt werden und zu Okklusions-, Muskel- und Kiefergelenkveränderungen führen (Bernhardt et al. 1999; Costa et al. 2012). 
Systemische Ursachen: Die CMD kann ihren Ursprung ebenfalls in einer Störung des Gesamtorganismus haben. Es besteht eine enge Beziehung zwischen dem Bewegungsapparat und dem Kauorgan (Ahlers 1998). Wirbelsäulenfehlstellungen oder eine unphysiologische Haltung können das Kausystem beeinflussen. Da der Unterkiefer nicht fest mit dem Schädel verbunden ist, bewegt er sich im entspannten Zustand immer in die Richtung, in die der Kopf geneigt ist. So können bei einer Körperfehlhaltung Vorkontakte oder - durch die Kompensationsarbeit der Muskulatur hervorgerufene - Muskelhypertrophien entstehen.

CMD-Schmerzen können aber auch durch den Organismus ausgelöst werden, ohne direkt mit dem Kauorgan in Verbindung zu stehen. Es handelt sich dabei um neurologische Entzündungen in peripheren Strukturen, die zu Schmerzen im Kiefergelenk und der angrenzenden Muskulatur führen. Auch die Trigeminusneuralgie fällt in diesen Ursachenbereich. Diese neurologischen Erkrankungen sind schwer zu diagnostizieren, da die Ursache nicht am Ort des Schmerzes liegt (Okeson und De Leeuw 2011).

Auch die genetische Disposition hat Einfluss auf die Empfindung von Schmerzen. Eine Variation auf dem COMT-Gen oder die reduzierte Verfügbarkeit des $\mu$-opioid-Rezeptors im linken Nucleus accumbens scheint nach Zubieta und DosSantos mit dem Auftreten von CMD und der chronischen Trigeminusneuralgie in Zusammenhang zu stehen (Zubieta et al. 2003; DosSantos et al. 2012).

Bei der CMD führt häufig nicht nur eine Ursache zur Erkrankung. Sie entsteht oftmals durch eine Akkumulation der Faktoren Bewegungsapparat, Kaumuskulatur, Kiefergelenk, Psyche, Zahnbett und Zahnhartsubstanzen (Ahlers 1998).

\subsubsection{Symptome}

Bei der CMD lassen sich im Wesentlichen subjektiv und objektiv wahrgenommene Symptome unterscheiden.

Objektive Symptome: Eines der hauptsächlich objektiven Symptome einer CMD ist die Abweichung der Unterkieferbewegung von der Norm. Dazu gehören Limitationen, Defle- 
xionen und Deviationen. Die Limitationen äußern sich durch eine eingeschränkte Mundöffnung. Dieser Zustand kann sich bei einer beidseitigen Diskusverlagerung ohne Reposition einstellen. Bei einer einseitigen Diskusverlagerung ohne Reposition weicht der Unterkiefer während der Mundöffnung zur betroffenen Seite hin ab. Diese Abweichung wird auch Deflexion genannt. Bei einer Deviation weicht der Unterkiefer bei der Mundöffnung zur erkrankten Seite hin ab, kehrt aber bei der weiteren Öffnungsbewegung zur Mittellinie zurück. Diese Bewegungsabweichung ist bei einer Diskusverlagerung mit Reposition gegeben (Ahlers 1998). Der Patient bemerkt diese Abweichungen in der Regel nur bei starker Ausprägung oder plötzlichem Auftreten der Bewegungseinschränkung beziehungsweise -abweichung.

Zu den objektiven Symptomen gehören auch die Palpationsbefunde der Kaumuskulatur, bei denen der Behandler Verhärtungen in Form von Myosen und Myogelosen ertasten kann (Strub et al. 2011). Diese Muskelveränderungen sind dem Patienten selbst meist nicht bekannt.

Ein weiteres Symptom der CMD ist das Kiefergelenkknacken (Mumford 1989). Dieses Geräusch im Bereich des Kiefergelenks ist in der Regel sowohl durch den Patienten als auch durch den Behandler wahrzunehmen. Neben einer Diskusverlagerung, Gelenkflächenveränderungen und Kondylusluxationen, kann es auch durch das Vorbeigleiten des lateralen Kondylenpols am Ligamentum Laterale entstehen (Lochmiller et al. 1991).

Subjektive Symptome: Neben den Kiefergelenkgeräuschen können auch Ohrgeräusche (Tinitus) auftreten (Kralijevic et al. 2003).

Das stärkste Symptom der CMD ist der Schmerz. Tritt eine CMD in Verbindung mit Schmerzsensationen auf, spricht man auch von einer Myoarthropathie (MAP) (Strub et al. 2011). Die MAP kann sich durch einen anhaltenden, ziehenden oder stechenden Schmerz im seitlichen Gesicht äußern. Der Schmerz kann den ganzen Tag andauern und über Wochen bis hin zu Monaten bestehen. Häufig beschreiben die Patienten eine variierende Intensität, die beim Aufwachen und/oder am Abend am stärksten ist. J.M. Mumford erklärt dieses Phänomen mit einer erhöhten Muskelaktivität über Nacht und einer allgemeinen Ermüdung am Abend. Darüber hinaus weist er auf die Ablenkung der Patienten über den Tag hinweg durch andere Aktivitäten hin. Laut Mumford kann es bei einigen Patienten außer- 
dem zu einem stechenden Schmerz in einer bestimmten Position des Kiefers kommen, der beispielsweise durch eine Diskusverlagerung mit schmerzhafter Kompression der bilaminären Zone ausgelöst wird (Mumford 1989; Okeson und De Leeuw 2011). Aus diesem Grunde führen einige Patienten eine Schonbewegung durch, die zwar eine abweichende Bewegung und manchmal auch ein Knacken verursacht, den Patienten aber von den Schmerzen befreit (Mumford 1989).

Schmerzen und Verspannungen können darüber hinaus in entfernteren Bereichen wie der Nackenregion auftreten. Die Nackenmuskulatur wird vermutlich herangezogen, um die ermüdete Kaumuskulatur zu unterstützen (Mumford 1989). Aus dieser verstärkten Belastung der Nackenmuskulatur, können sich sogenannte Spannungskopfschmerzen entwickeln (Detamore und Alhanasiou 2003; Kralijevic et al. 2003).

Wie stark ein Schmerz wahrgenommen wird, ist laut Dworkin von Mensch zu Mensch unterschiedlich und hängt unter anderem vom Erbgut ab. Darüber hinaus kann das Gehirn lernen und erinnern, was zu chronischen Schmerzen führen kann, die keinen direkten Auslöser mehr haben (Dworkin 2010).

Zusammenfassend lässt sich sagen, dass sich die CMD durch Bewegungsabweichungen, Muskelverhärtungen und -verspannungen, Geräusche im Kiefergelenk und Schmerzen äuBert.

\subsubsection{Hilfsmittel zur Feststellung einer CMD}

Da die Hintergründe der jeweiligen Symptomatik individuell sind, ist die Ursachenermittlung Grundlage für eine adäquate Therapie (Bernhardt und Schwahn 1999). Um den Auslöser für eine Funktionsstörung zu ermitteln, gibt es diverse Herangehensweisen und Systeme. Es stehen dem Behandler das Anamnesegespräch, die klinische Befunderhebung und Röntgenanalyse sowie die klinische und instrumentelle Funktionsanalyse zur Verfügung.

Anamnese: Die allgemeine Befragung schließt Fragen zum Gesundheitszustand, der Medikamentennahme, der psychischen Belastung und Schmerzen ein. Dafür wird dem 
Patienten ein Anamnesebogen mit gezielten Fragen gegeben, den dieser selbstständig ausfüllt. Zum Behandlungsbeginn bespricht der behandelnde Arzt den Bogen zusammen mit dem Patienten und geht auf bestimmte Fragen noch einmal gezielt ein.

Klinische Befundung und Röntgenanalyse: Die klinische Befundung schließt den dentalen sowie den Schleimhautbefund ein. Darüber hinaus sollte ein Parodonal-Status erhoben werden (Siebert 1987). Bei der Befundung der röntgenologischen Kiefergelenkdarstellungen weist Hanel auf die großen Schwierigkeiten bei der Interpretation der Bilder hin, da diese durch Überlagerungen falsche Schlüsse zulassen (Hanel 1984).

Klinische Funktionsanalyse: Bei der klinischen Funktionsanalyse wird der funktionelle Zustand und das Zusammenwirken der Zähne, der Muskulatur und der Kiefergelenke überprüft (Ahlers et al. 2003a). Es wird die Mobilität des Unterkiefers gemessen (Schneidekantendistanz (Siebert 1987), Laterotrusionswerte, Mittellinienabweichung) und die Kiefergelenke palpiert und auskultiert (mit /ohne Stethoskop). Des Weiteren werden die Muskeln bilateral palpiert und die Zunge, die Lippen und die Wangen auf Impressionen untersucht. Darauf folgt die Untersuchung der statischen und dynamischen Okklusion (Hanel 1984).

Laut Stellungnahme der DGZMK zur klinischen Funktionsanalyse ist das Erkennen von funktionellen Störungen und Erkrankungen ohne funktionsanalytische Maßnahmen nicht möglich. Die klinische Funktionsanalyse ist dabei für die Untersuchung von Patienten mit Craniomandibulären Dysfunktionen als grundlegend anzusehen (Ahlers et al. 2003b). Die klinische Funktionsanalyse bildet den ersten Teil der funktionellen Untersuchung auf dessen Grundlage über die Notwendigkeit weiterer Untersuchungsverfahren (instrumentelle Funktionsanalyse) entschieden wird (Ahlers et al. 2003b).

Instrumentelle Funktionsanalyse: Die instrumentelle Funktionsanalyse erfasst den Funktionszustand des stomatognathen Systems mit Hilfe von speziellen Apparaturen (Gesichtsbogen). Die Geräte dokumentieren die dreidimensionale Unterkieferbewegung einschließlich der Kondylenbewegung durch Messdaten (Achsiographie), um den aktuellen Funktionszustand beschreiben und analysieren zu können. Die Aufzeichnung kann mit 
mechanischen Geräten durchgeführt werden, sofern diese gelenknah ausgerichtet sind. Die Aussagen dieser Messgeräte bei einer Bewertung der Gelenkdynamik sind jedoch limitiert (Engelhardt et al. 2002). Moderne Systeme arbeiten auf elektronischer, optoelektronischer, elektromagnetischer oder Ultraschall-Basis und einer elektronischen Datenverarbeitung (Engelhardt et al. 2002). Die Bewegungsaufzeichnung und -analyse mit Hilfe eines elektronischen Gesichtsbogens hat in den letzten Jahrzehnten an Bedeutung gewonnen. Die Graphen dienen neben der Analyse auch der Dokumentation (Ellerbrock 2009).

Erst nach der Durchführung einer eingehenden Untersuchung ist die Formulierung einer Diagnose zulässig. Diese erlaubt es, einen adäquaten Therapieplan aufzustellen (Ahlers 1998).

Um eine einheitliche Bewertung der CMD-Symptome zu erreichen und somit gleiche Ergebnisse in der Diagnose zu erhalten, wurden im Jahre 1992 die Research Diagnostic Criteria for Temporomandibular Disorders (RDC/TMD) eingeführt. Diese Kriterienliste kann für die Untersuchung, die Diagnose und die Klassifizierung der meisten CMD-Untergruppen genutzt werden (Manfredini et al. 2011).

\subsubsection{Diagnose}

Das Krankheitsbild der CMD umfasst verschiedene Symptome, die auch kombiniert auftreten können. Um ein standardisiertes Verfahren für die Untersuchung, Diagnose und Klassifizierung der Subtypen der CMD-Erkrankungen zu erhalten, wurde 1992 von einer Expertengruppe um Dworkin und LeResche eine Klassifikation der CMD erstellt, die $R D C / T M D$. Diese Diagnosekriterien sollten internationale Verwendung finden und wurden dafür in 20 Sprachen übersetzt (Dworkin 2010). Die Diagnosen werden anhand von standardisierten Fragebögen und klinischen Untersuchungen getroffen.

Die Einteilung erfolgt in zwei Achsen. Achse I beinhaltet die klinische CMD-Diagnostik, Achse II umfasst den psychosozialen Status des Patienten (Lobbezoo et al. 2004). 


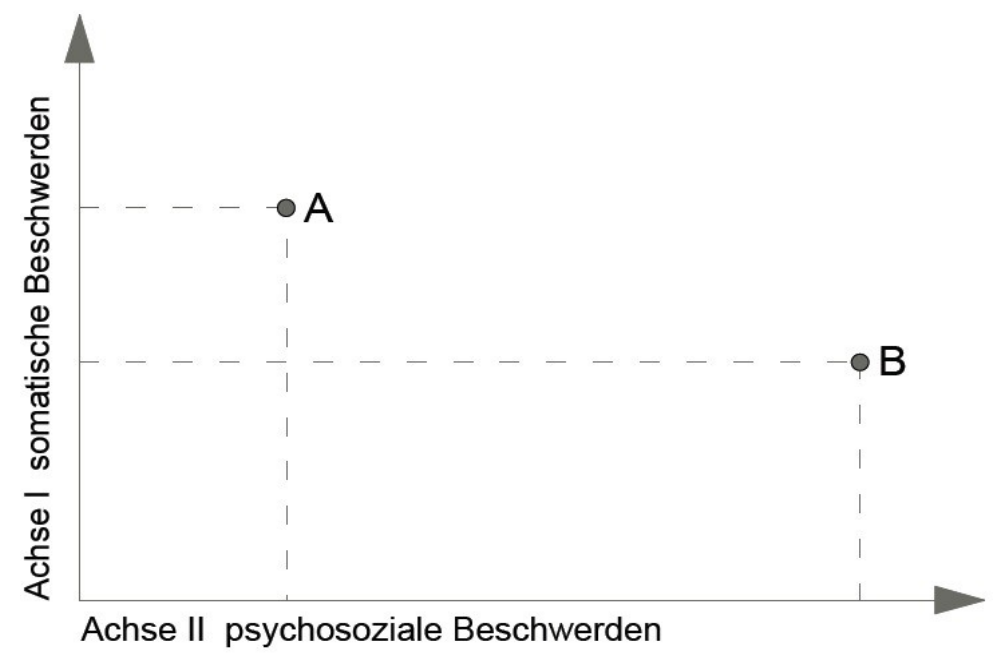

Abb. 1: Achse I und Achse II; Patient A: starke somatische Beschwerden bei geringer psychischer Belastung; Patient B: starke psychische Belastung bei geringen somatischen Beschwerden.

Die erste Achse lässt sich in die drei Gruppen Muskelstörung (I), Diskusverlagerung (II) und Arthralgie/Arthritis/Arthrose (III) aufteilen (Lobbezoo et al. 2004). Diese Gruppen lassen sich noch weiter untergliedern (Strub et al. 2011):

Gruppe I: Schmerzhafte Beschwerden im Bereich der Kiefermuskulatur

I.a Myofazialer Schmerz

I.b Myofazialer Schmerz mit eingeschränkter Kieferöffnung

Gruppe II: Verlagerungen des Discus articularis

II.a Diskusverlagerung mit Reposition bei der Kieferöffnung

II.b Diskusverlagerung ohne Reposition bei der Kieferöffnung, mit eingeschränkter Kieferöffnung

II.c Diskusverlagerung ohne Reposition bei der Kieferöffnung, ohne eingeschränkte Kieferöffnung

Gruppe III: Arthralgie, Arthritis, Arthrose

III.a Arthralgie des Kiefergelenks

III.b Arthritis des Kiefergelenks

III.c Arthrose des Kiefergelenks 
Eine eindeutige Diagnose anhand der bestehenden Symptome ist nicht immer möglich. Durch verschiedene Methoden der Untersuchung (Art der Palpation, Druckausübung, Auskultation mit/ohne Stethoskop, Verwendung verschiedener Idealwerte für die Mundöffnung) und die Veränderung der Beschwerden und Symptome innerhalb kurzer Zeit, weisen die Untersuchungen zum Teil inkonstante Ergebnisse auf (Strub et al. 2011).

In einer Untersuchung, durchgeführt im Zusammenschluss von Universitäten aus vier verschiedenen Ländern, wurde die Handhabung der Research Diagnostic Criteria for Temporomandibular Disorders (RDC/TMD) in verschiedenen Studien untersucht. Insgesamt haben mehr als 3.000 CMD-Patienten an den einbezogenen Studien teilgenommen. Die Studie hat aus den Untersuchungen folgende Ergebnisse erhalten (Manfredini et al. 2011):

Myofazialer Schmerz mit oder ohne limitierte Mundöffnung ist die häufigste Achse-I-Diagnose (45,3 \%, Gruppe I). 41,5 \% der Patienten waren von einer Diskusverlagerung mit Reposition betroffen (Gruppe II). Bei etwa einem Drittel der Patienten (30,1\%) wurden entzündlich-degenerative Prozesse festgestellt (Gruppe III), wobei bei 34,2 \% dieser Personen eine Arthralgie als Diagnose gestellt wurde. In der allgemeinen Bevölkerung leiden 6 - $12 \%$ an myofazialen Schmerzen und 8,9 - 15,8 \% an einer Diskusverlagerung mit Reposition.

Eine Studie an einer norditalienischen Klinik mit 243 untersuchten Patienten kam zu dem Ergebnis, dass 49,7 \% der untersuchten Patienten der Gruppe I der RDC/TMD zuzuordnen war. 57,3 \% hatten Diskusverlagerungen (Gruppe II) und 81,4 \% wiesen GruppeIII-Erkrankungen (Arthralgie, Arthritis, Arthrose) auf. 64,3 \% der Patienten hatten mehr als eine Gruppenzugehörigkeit (Manfredini et al. 2010).

Es konnte des Weiteren eine Altersabhängigkeit in der Gruppenzuordnung ausgemacht werden. Eine Gruppe im Alter von $32 \pm 14,5$ Jahren zeigte hauptsächlich Diskusverlagerungen und eine zweite Gruppe im Alter von 54,2 \pm 15,1 Jahren wies in erster Linie entzündlich-degenerative Prozesse im Kiefergelenk auf. Die verschiedenen Erkrankungen des Kiefergelenks zeigen also altersabhängige Höchstwerte (Manfredini et al. 2010).

Der Vergleich verschiedener Studienergebnisse ist oft nur bedingt möglich, da die Research Diagnostic Criteria for Temporomandibular Disorders (RDC/TMD) eher selten als Standard für die Studienauswertung herangezogen werden (Manfredini et al. 2011).

Darüber hinaus nehmen individuelle Untersuchungsmethoden und Einschätzungen des 
Behandlers Einfluss auf die Klassifizierung der CMD-Erkrankung (Strub et al. 2011).

\subsubsection{Therapie}

Der erste Schritt in der Behandlung einer CMD ist es, Vertrauen zum Patienten aufzubauen und beruhigend auf diesen einzuwirken (Mumford 1989). Viele Patienten leiden unter den Symptomen der Erkrankung oder haben Angst vor den Ursachen und der weiteren Entwicklung. Aus diesem Grund sollte der Behandler den Patienten über die Ursachen, Folgen und die Prävalenz der Erkrankung aufklären und den Patienten somit auch auf psychologischer Ebene entlasten.

Da es sich bei der CMD um eine multifaktorielle Erkrankung handelt, muss eine genaue Ursachenforschung betrieben werden, um einen adäquaten Therapieplan zu erstellen. Die Therapie sollte daraufhin in Stufen durchgeführt werden, beginnend mit nichtinvasiven Maßnahmen.

Zunächst wird der Patient aktiv eingebunden und zur Selbstbeobachtung animiert. Bestimmte Parafunktionen (Knirschen, Pressen, Wangenbeißen) können vom Patienten wahrgenommen werden und im günstigsten Fall bewusst vermindert oder sogar verhindert werden (Arnholz 1994).

Stressabbauende Aktivitäten wie Sport und ein kurzer Mittagsschlaf sind außerdem Spannungslöser. Darüber hinaus sollte der Patient auf seinen Koffeinkonsum achten und die Menge gegebenenfalls reduzieren (Mumford 1989).

Auch Massagen im Kopf- und Nackenbereich wirken spannungslösend und fördern die Durchblutung. Sie können vom Patienten selbst oder durch geschultes Personal durchgeführt werden (Hanel 1984).

Ein weiterer Schritt in der Behandlung ist die Physiotherapie. Die Muskulatur wird mit Hilfe von Bestrahlungsgeräten, Reizstromgeräten, Wärme- und Kältebehandlungen entspannt (Hanel 1984). Der Patient kann dabei mit erlernten Übungen zur Muskelentspannung beitragen.

Bei stressbedingtem Knirschen und Pressen, welches meist nachts und unbewusst stattfindet, kann zur unterstützenden Therapie die Gabe von muskelrelaxierenden Medikamenten indiziert sein (Arnholz 1994; Hanel 1984; Mumford 1989). 
Wenn die Beschwerden nach 14 Tagen nicht abklingen, wird eine Aufbissschiene zur Entlastung von Kiefergelenk und Kaumuskulatur angefertigt. Mit einer solchen Schiene werden Kiefergelenke und Muskulatur entspannt und dem Patienten eine physiologische Zentrik gegeben (Hanel 1984).

Wenn auch eine Aufbissschiene nicht den gewünschten Erfolg bringt, muss eine kritische Diagnoseprüfung sowie eine interdisziplinäre Ursachenforschung erfolgen. Eine mögliche weitere Therapiemaßnahme wäre eine psychotherapeutische Behandlung (Arnholz 1994; Hanel 1984). Darüber hinaus können am Ende der Behandlung auch invasive Schritte, wie das selektive Beschleifen der Zähne sowie konservierende, prothetische oder kieferorthopädische Maßnahmen stehen, die die Ursache einer Dysfunktion beheben (Arnholz 1994; Mumford 1989).

Einige CMD-Symptome, wie zum Beispiel Kiefergelenkgeräusche, werden heute weniger oft als behandlungsbedürftig angesehen als noch vor 20 Jahren (Al-Jundi et al. 2008).

Eine Untersuchung, die verschiedene Studien zum Thema „Treatment Need for Temporomandibular Disorders in Adult Nonpatients“ vereint hat, kam zu dem Ergebnis, dass bei rund $16 \%$ der 9.454 erfassten Personen die Notwendigkeit einer CMD-Behandlung vorlag. Dies unterstreicht die Wichtigkeit der Erkrankung in der allgemeinen Gesundheit. Außerdem wurde aufgezeigt, dass die Einschätzung von Behandler und Patienten, was die Behandlungsbedürftigkeit der CMD angeht, auseinander liegen. Die Patienten stuften sich selbst häufiger als „nicht behandlungsbedürftig“ ein, als es der Arzt nach Durchführung einer klinischen Untersuchung tat (Al-Jundi et al. 2008).

\subsection{Instrumentelle Funktionsanalyse}

\subsubsection{Beschreibung}

Die instrumentelle Funktionsanalyse einschließlich der elektronischen Vermessung ist ein Verfahren zur Erfassung des Funktionszustandes des craniomandibulären Systems.

Zielsetzung der Anwendung ist es, mit Hilfe von Messgeräten ein besseres Verständnis der Biomechanik der Kiefergelenke zu erlangen, mögliche Fehlfunktionen zu diagnostizie- 
ren, geeignete Therapiemaßnahmen zu ermitteln und den Therapieerfolg zu überprüfen (Ahlers et al. 2003a; Ellerbrock 2009; Schmitter et al. 2011).

Durch elektronische Messungen lassen sich die Bewegungen der Kiefergelenke und des Inzisalpunktes aufzeichnen. Anhand dieser Werte kann der Behandler Aussagen zu gegebenen Limitationen, Asymmetrien und Versprüngen treffen. Die Aufzeichnungen können des Weiteren zur neuromuskulären Diagnostik eingesetzt werden (Ellerbrock 2009).

Darüber hinaus soll durch die Verwendung der patientenspezifischen Gelenkparameter im Artikulator bei der Anfertigung von Zahnersatz die intraorale Anpassung minimiert werden (Schierz und Reißmann 2008). Es können dafür unter anderem der sagittale Kondylenbahnneigungswinkel, der Bennettwinkel, der Immediate-side-shift und die Frontzahnführung aufgezeichnet werden (Ellerbrock 2009; Lehmann et al. 2009).

Nach Bumann ist der entscheidende Unterschied der instrumentellen Funktionsanalyse zur klassischen klinischen Funktionsanalyse, dass auch bei anamnestisch symptomlosen Patienten noch „versteckte“ (kompensierte) Funktionsstörungen diagnostiziert werden können (Bumann 2007).

\subsubsection{Indikationen}

Die Deutsche Gesellschaft für Zahn-, Mund- und Kieferheilkunde (DGZMK) teilt die Indikation für eine instrumentelle Funktionsanalyse in vier Gruppen ein:

Zur ersten Gruppe gehören „Zahn-, Kiefergelenk- und Muskelerkrankungen bei Verdacht auf Okklusionsstörung“, „Kiefergelenk- und Muskelerkrankungen, die mit stark von der Norm abweichenden Gelenkbewegungen verbunden sind“ sowie „Kiefergelenk- und Muskelerkrankungen bei Vorliegen von Dysgnathien“. Die zweite Gruppe umfasst die Funktionsanalyse bei umfangreichen restaurativen und prothetischen Versorgungen zur Rekonstruktion und Erhaltung des Gebisses. Die Gruppe drei beinhaltet die funktionelle Vorbehandlung des craniomandibulären Systems bei Diagnostik und Operationsplanung im Rahmen kieferorthopädischer und/oder kieferchirurgischer Behandlungen. Und die vierte Gruppe bezieht sich auf Parodontopathien, wenn Hinweise auf Fehlbelastungen der Zähne bestehen (Ahlers et al. 2003a). 


\subsubsection{Messprinzipien}

Die Funktionsdiagnostik nutzt die Apparaturen zur Unterkieferaufzeichnung für die Ermittlung therapierelevanter diagnostischer Informationen über das stomatognathe System (Schmitter et al. 2011).

Man unterscheidet zwei grundlegende Messprinzipien: die gelenknahe Aufzeichnung in enger Lagebeziehung zum Kiefergelenk und die gelenkferne Aufzeichnung, bei der in der Regel ventral des Patientengesichts gemessen wird. Da die Anbringung von Messgeräten am Kiefergelenk selbst nicht möglich ist, müssen alle Sensoren gelenkfern - beispielsweise am Zahnbogen - fixiert werden. Diese Tatsache erschwert die Beurteilung der Kondylenbewegung erheblich (Schierz und Reißmann 2008).

Je nach individueller Schmerzlage des Patienten können verschiedene Kondylenpositionen aufgezeichnet werden. Damit kann der Behandler ermitteln, ob die Funktionsstörung in einem direkten Zusammenhang mit der statischen oder der dynamischen Okklusion, einem bestimmten Bruxismusmuster oder einer anderen Dysfunktion steht (Bumann 2007).

\subsection{Elektronische Systeme zur Unterkieferaufzeichnung}

Da sich die vorliegende Studie auf elektronische Messsysteme bezieht, soll im weiteren Verlauf der Arbeit nur auf diese eingegangen werden.

\subsubsection{Gelenknahe Aufzeichnung}

$\mathrm{Zu}$ den in unmittelbarer Nähe der Kiefergelenke aufzeichnenden Systeme gehören die Elektromechanischen sowie die Optoelektronischen Registriersysteme. Da die Geräte nicht direkt im Bereich der Kiefergelenke befestigt werden können, werden die Registrierbögen bei der gelenknahen Aufzeichnung in der Regel intraoral an den Zähnen oder dem Zahnersatz fixiert (Schierz und Reißmann 2008). Die Bögen haben neben dem intraoral befestigten Teil einen extraoralen Anteil, der bis zu den Kiefergelenken reicht. Hier erfolgt die Messung der Kiefergelenkbewegungen. 


\subsubsection{Elektromechanische Registriersysteme}

Die auf elektromechanischer Basis arbeitenden Systeme bestehen aus einem maxillären und einem mandibulären Gesichtsbogen. An dem Oberkieferbogen wird in sagittaler Ausrichtung ein Sensortablett im Bereich der Kondylen befestigt. Am Unterkieferbogen, auch Schreibbogen genannt, werden im Bereich der Kiefergelenke Taststifte angebracht. Die Position der Stifte kann variiert werden, um sie an die individuelle Lage der Scharnierachsen anzupassen. Bei der Bewegung des Unterkiefers wird die Bewegungsbahn durch den Kontakt zwischen den Taststiften und dem Sensorblatt elektronisch aufgezeichnet.

Elektromechanische Registriersysteme dieser Art sind das System CARDIAX® Compact 2 beziehungsweise 4 und CARDIAX® Diagnostic der Firma Gamma, sowie die Systeme AXIOGRAPH $®$ und AXIOTRON $®$ des Unternehmens SAM Präzisionstechnik. Das Zusatzgerät AXIOTRON® bietet dem Anwender die Möglichkeit, die Bewegungen hinsichtlich des zeitlichen Ablaufs und der Beschleunigungen auszuwerten (Schierz und Reißmann 2008).

\subsubsection{Optoelektronische Registriersysteme}

Optoelektronische Systeme können sich in ihrer Konstruktion wesentlich voneinander unterscheiden. Dies kann anhand zweier Systeme der Firma Dentron deutlich gemacht werden.

Das Gerät CONDYLOCOMP LR3® besteht aus einem maxillären und einem mandibulären Gesichtsbogen und basiert auf einem Lichtreflexionsprinzip. Am oberen Kopfteil sind Infrarotsensoren angebracht, die in drei Richtungen des Raumes Licht emittieren und reflektierte Lichtimpulse empfangen. Reflektoren, die an den kiefergelenknahen Enden des Unterkieferbogens befestigt sind, werfen das Licht zu den Oberkiefersensoren zurück. Die Registrierung erfolgt berührungsfrei. Es sind also keine Kabelverbindungen zwischen dem maxillären und mandibulären Bogen nötig (Schierz und Reißmann 2008).

Das Registriersystem Freecorder BlueFox® ist das Nachfolgemodell der Firma Dentron. Es besteht ebenfalls aus einem maxillären und mandibulären Bogen. Die Einheit zur optischen Erfassung ist hier aber nicht am Schädel des Patienten angebracht, sondern bildet - ähnlich eines Panorama-Röntgengerätes - eine stationäre Einheit, die den Kopf des Patienten umgibt (Schierz und Reißmann 2008). Für die Bewegungsaufzeichnung befin- 
den sich Schwarz-Weiß-Muster an den Enden von Gesichts- und Unterkieferbogen. Extraoral angeordnete Spezialkameras verfolgen die Schwarz-Weiß-Tafeln via Muster-Tracking (Ellerbrock 2009). Durch die Notwendigkeit mehrerer Kameras und die stationäre Einrichtung des Systems, betragen die Anschaffungskosten ein Mehrfaches der anderen auf dem Markt vertretenen Systeme (Schmitter et al. 2011).

\subsubsection{Gelenkferne Aufzeichnung}

Da im Bereich der Kiefergelenke keine sichere Fixierung von Aufzeichnungs- beziehungsweise Messgeräten möglich ist, müssen diese gelenkfern an den Zähnen oder dem Zahnersatz befestigt werden (Schierz und Reißmann 2008). Die Messung der gelenkfern arbeitenden Systeme erfolgt immer berührungslos und in der Regel vor dem Gesicht des Patienten, was dazu führt, dass die Scharnierachsposition und deren Bewegung aus den gewonnenen Daten errechnet werden muss. Dies erfordert eine hohe Präzision der Datenaufzeichnung, da sich Messfehler bei der Berechnung potenzieren können (Schierz und Reißmann 2008).

Die gelenkferne Messung kann mit Ultraschall oder mit Hilfe von Magnetkinesiografie durchgeführt werden. Auf dem Markt erhältliche Geräte arbeiten aber nahezu ausschließlich auf Ultraschallbasis.

\subsubsection{Ultraschallbasierte Registriersysteme}

Ultraschallmessgeräte bestehen aus einem maxillären und einem mandibulären Gesichtsbogen. An einem der Bögen sind Sender angebracht, die Ultraschallsignale aussenden. An dem anderen Bogen befinden sich Empfänger, die die Signale aufnehmen. Durch die Berechnung der Zeitintervalle, die die Ultraschallsignale benötigen, um vom Sender zum Empfänger zu gelangen, kann der zurückgelegte Weg der Signale errechnet und somit die Position des Unterkieferbogens im Verhältnis zum Oberkieferbogen ermittelt werden. An den Gesichtsbögen sind jeweils mehrere Sender beziehungsweise Empfänger angebracht, wodurch mehrere Signale zur gleichen Zeit registriert werden können. Dadurch ist es möglich, die Bewegung des Unterkiefers im dreidimensionalen Raum aufzuzeichnen 
und Messfehler zu reduzieren (Schierz und Reißmann 2008).

Ultraschallmessgeräte sind unter anderem der AXIOQUICK® Recorder der Firma SAM Präzisionstechnik, das System Jaw Motion Analysis der Firma Zebris und der ARCUS®digma der Firma KaVo Dental (Schierz und Reißmann 2008). Bei dem System der Firma Zebris besteht neben der Ultraschallmessung die Möglichkeit ein Elektromyogramm aufzuzeichnen. Außerdem kann mittels 2-Kanal-Gelenkgeräusch-System die Frequenz und Schallintensität von Gelenkgeräuschen ermittelt werden (Schmitter et al. 2011). Eine Sonderform der Ultraschallmessung ist die Ultraschalltopometrie. Es werden dafür Ultraschallsender auf der Haut oder an einem paraokklusalen Löffel befestigt. Diese senden im Takt weniger Millisekunden Ultraschallimpulse an fest im Raum installierte Empfänger. Mit Hilfe der Empfänger und einer speziellen Software können die Koordinaten des Senders im dreidimensionalen Raum berechnet werden. Mit einem vierten Empfänger kann die Richtung der Einzelmessungen überprüft werden (Arnholz 1994).

\subsubsection{Magnetkinesiografie}

Ein Beispiel für die Anwendung der Magnetkinesiografie ist das K7-Modul der Firma SinfoMed, welches auf der Magnetfeld-Messmethode des Sirognathographen nach Lewin (1972) basiert. Ein Magnet wird vestibulär an der Unterkieferfront befestigt. Dessen Verlaufsbahnen werden während der Bewegung des Unterkiefers von einem am Kopf fixierten Außenbogen gemessen und durch eine Software aufgezeichnet (Wegmann 2013). Der Magnet wiegt $2 \mathrm{~g}$ und der Außenbogen 180g. Durch das geringe Gewicht können die Unterkieferbewegungen störungsfrei durchgeführt werden. Die Bewegungsbahnen des Magneten werden dreidimensional aufgezeichnet und am Computerbildschirm dargestellt (Sinfomed 2014).

Ein weiteres Beispiel ist der Sirognathograph der Firma Siemens, welcher aber heute nicht mehr auf dem Markt erhältlich ist. Bei diesem Gerät wird zunächst die Muskulatur des Patienten durch transkutane elektrische Neurostimulation entspannt. Auch hier wird ein Magnet auf der Vestibularfläche der unteren Inzisivi befestigt und die Bewegungen mit Hilfe eines am Kopf fixierten Außenbogens aufgezeichnet (Schmitter et al. 2011).

Ein Nachteil der magnetischen Methoden ist, dass sie sensibel auf metallische Gegen- 
stände in der Umgebung reagieren können und somit Verfälschungen der Aufzeichnungen nicht auszuschließen sind (Furtado et al. 2013). Der Vorteil der Magnetkinesiografie ist, dass kein Unterkieferbogen benötigt wird und die Unterkieferbewegungen uneingeschränkt durchgeführt werden können (Ellerbrock 2009).

Die Magnetkinesiografie ist auf dem Markt weniger vertreten, als ultraschallbasierte Registriersysteme. Studien zeigen aber, dass weiterhin an dieser Technik gearbeitet und geforscht wird (Baeyens et al. 2013; Yoon et al. 2006).

\subsection{Entwicklung der elektronischen und optoelektronischen Registriersysteme}

\subsubsection{Optoelektronische Registriersysteme}

Aus der fotografischen Methode der Unterkieferaufzeichnung haben sich mit Etablierung der Filmkamera die Filmanalyse und mit der Einführung von Computern mit entsprechender Software die optoelektronischen Registriersysteme entwickelt.

Optische Methoden zur Unterkieferbewegungsanalyse haben also eine lange Tradition. Schon im Jahre 1889 wurden von Luce Untersuchungen mit Hilfe von Reihenfotografien durchgeführt. Es wurden dabei Silberperlen an den Unterkieferinzisivi und einem extraoralen Gesichtsbogen befestigt (Luce 1889). Thouren führte im Jahre 1914 die Methode der Kameraaufnahme (16 Bilder pro Sekunde) ein. Bei dieser Untersuchung standen die Rotationsachsen des Unterkiefers im Vordergrund (Thouren 1914). 1926 wurden von Munzesheimer durch den Gebrauch mehrerer Fotoapparate dreidimensionale Aufnahmen erzeugt (Munzesheimer 1928). Hildebrand war es 1931 möglich, mit Zuhilfenahme von Spiegeln, die im $45^{\circ}$ Winkel am Kopfteil befestigt wurden, mit nur einer Filmkamera, eine dreidimensionale Darstellung zu erreichen. Darüber hinaus berechnete er die Geschwindigkeit der Kaubewegungen (Hildebrand 1931). Im Laufe der Zeit wurden die Analysemethoden von verschiedenen Wissenschaftlern weiterentwickelt (Atkinson und Shepherd 1955; Shepherd 1960; Woelfel et al. 1962; Beyron 1964; Ahlgren 1967). Im Jahre 1967 präsentierte Gillings seinen Photoelektronischen Mandibulographen. Dieser Apparat bestand aus einem Unterkiefergestell, einer Lichtquelle, welche auf der Labialfläche der unteren Inzisivi fixiert wurde und Photozellen, die auf einem extraoralen Rahmen angebracht waren. Das System 
nahm Bewegungen in drei Dimensionen durch die Positionsänderung der Lichtquelle war (Gillings 1967).

Ein weiteres optoelektronisches System wurde von Karlsson im Jahre 1977 beschrieben. Er benutzte bei seinen Aufzeichnungen zwei Licht-emittierende Dioden (LED), die Infrarotlicht aussendeten. Die eine Bewegungsdiode wurde am Kinn und eine Referenzdiode an der Stirn befestigt, um die Kopfbewegungen von den Unterkieferbewegungen abzuziehen. Darüber hinaus benutzte Karlsson zwei Kameras, um die dreidimensionalen Koordinaten der Bewegungen zu berechnen (Karlsson 1977). 1982 untersuchten Jemt und Hedegard, ob die Anbringung eines Markers auf der Kinnhaut zu Verfälschungen der Bewegung führt. Sie verglichen die Bewegungsbahnen des Kinnmarkers mit denen eines an den unteren Inzisivi angebrachten Markers und stellten fest, dass die Kinnreferenz für ihren untersuchten Kaurhythmus eingesetzt werden konnte (Jemt und Hedegård 1982). Auch Atkinson und Shepherd benutzten den Kinnpunkt im Jahre 1955 als Bewegungspunkt. Damals wurde diese Messmethode allerdings für nicht geeignet befunden (Atkinson und Shepherd 1955). 1985 entwickelten Mesqui und Palla das System JAWS-3D®. Dieses System bestand aus drei Kameras, die die Position von sechs Leuchtdioden aufzeichneten. Die Lichtquellen waren an Metallrahmen angebracht, die ihrerseits an den Oberkiefer- und Unterkieferzahnbögen befestigt waren (Mesqui und Palla 1985).

Im Jahre 1996 wurde das optoelektronische System „Mac Reflex“ von Hamborg\&Karlsson beschrieben. Es besteht aus zwei Videokameras, einem Videoprozessor und einem Software-Paket. Infrarotlichter sind um die Linse herum angebracht und an den Probanden befestigte Reflektoren werfen dieses Licht zur Kamera zurück (Hamborg und Karlsson 1996). In ihrem „Historical review of methods development" kommen Soboleva et al. zu dem Schluss, dass optoelektronische Geräte weniger störenden Einfluss auf die Unterkieferbewegungen der Probanden nehmen als vergleichbare Instrumente anderer Messsysteme (Soboleva et al. 2005 b). 


\subsubsection{Elektronische Registriersysteme}

1953 wurde von Jankelson eine elektronische Methode zur Frequenzmessung von Zahnkontakten vorgestellt (Jankelson et al. 1952). Auch Yurkstas und Emerson, Brewer und Hudson, Graf und Zander und Neill arbeiteten auf ähnliche Art und Weise (Yurkstas und Emerson 1954; Brewer und Hudson 1961; Graf und Zander 1963; Neill 1967). Alle benutzten einen Stromkreis, der sich bei Zahnkontakt schloss und diesen Befund grafisch als Kurvenauslenkung oder in Form eines Lichtsignals wiedergab (Soboleva et al. 2005b).

Glickman et al. entwickelten die bestehenden Methoden weiter und beschrieben 1968 ein System, das drei verschiedene okklusale Positionen differenzieren konnte (Glickman et al. 1968). 1969 wurden die Kontaktmessungen vom gleichen Arbeitsteam auf fünf ausgeweitet (Glickman et al. 1969).

1967 wurde von Bewersdorff eines der ersten dreidimensional arbeitenden elektronischen Registriersysteme entwickelt. Das Verfahren zur Unterkieferbewegungsaufzeichnung wurde als "Elektrognathographie" bezeichnet. Die Apparatur wurde über ein Seilsystem vor dem Patienten aufgehängt und über eine Bissgabel intraoral befestigt. Über Winkelmessungen von Drähten konnten die Bewegungen dokumentiert und gespeichert werden (Bewersdorff 1970). Ebenfalls 1967 präsentierte T. Messerman ein elektronisches Registriersystem, den Case-Gnathic-Replicator. Von Knap wurde im Jahre 1970 ein dreidimensionales Registriersystem entwickelt, welches die Unterkieferbewegung mit Hilfe von sechs Potentiometern aufzeichnete (Knap et al. 1970a und b).

1975 wurde von Johannsen und Goodson der Mandibulograph vorgestellt. Es war das erste Verfahren, welches mit Hilfe eines Computers die mathematische Bestimmung der räumlichen Unterkieferbewegungsbahn berechnete. Für die Registrierungen musste der Kopf des Probanden mit einer Gipsschale fixiert werden, um äußere Bewegungseinflüsse zu verhindern (Goodson und Johansen 1975). Im Jahre 1978 präsentierte Ohlrogge ein System, bei welchem die Signalgeber gelenknah befestigt und die Sensoren in den Behandlungsstuhl integriert waren (Simonet 1981). 1981 stellten Körber und Luckenbach ihr ECRS-System vor, welches jede beliebige Position eines Punktes im Kiefer relativ zur Messapparatur bestimmen konnte. Möglich war dies mit Hilfe von sieben Drehpotentiometern, welche die dreidimensionalen Ober- und Unterkieferpositionen eines Gipsmodells im Artikulator darstellen konnten (Körber und Luckenbach 1981). Hobo und Mochizuki veröf- 
fentlichten 1983 ein Gerät, mit welchem Unterkieferbewegungen in sechs Freiheitsgraden mit einer Genauigkeit von $\pm 0,06 \mathrm{~mm}$ gemessen werden konnten (Hobo und Mochizuki 1983). Im Jahre 1986 wurde dann von Hans-Ulrich Häring die computergestützte Ultraschallaufzeichnung entwickelt (Schierz und Reißmann 2008). Den von inm entwickelten Elektrognathographen benutzte Pröschel 1987, um die Kaubewegung in verschiedene Kaubewegungsmuster zu untergliedern (Pröschel 1987a und b). Handel führte 1987 Untersuchungen zum zeitlich-räumlichen Vergleich beider Kiefergelenke durch (Engelskirchen 1998).

Auf Grundlage der Ultraschalltechnik wurden im Laufe der Zeit Systeme wie der AXIOQUICK® Recorder der Firma SAM Präzisionstechnik, das System Jaw Motion Analysis der Firma Zebris und der ARCUS®digma der Firma KaVo Dental entwickelt (Schierz und Reißmann 2008). Der ARCUS®digma wurde im Jahr 2007 auf den Markt gebracht. Bei diesem Gerät werden die Unterkieferbewegungen in Echtzeit aufgezeichnet und sind digital abrufbar. Der Gesichtsbogen kommt sowohl im funktionsdiagnostischen als auch im konservierenden und prothetischen Bereich zum Einsatz.

\subsection{Fragestellung und Zielsetzung}

Auf dem Markt ist ein breites Feld an Apparaturen zur Registrierung des Funktionsstatus zu finden. Diese Geräte bringen allerdings mehrere Probleme mit sich.

Zum Beispiel sind die Anschaffungskosten der Apparaturen sehr hoch, was den Einsatz in den meisten Praxen verhindert. Darüber hinaus ist die Anbringung der Geräte häufig zeitaufwendig und für die Anwendung im klinischen Alltag bedarf es einer Einweisung und Einarbeitung in die Funktionsweise der Apparaturen, sowie der zugehörigen Software. Viele Geräte haben des Weiteren ein hohes Gewicht und die invasive Anbringung kann den natürlichen Bewegungsablauf behindern.

In Anbetracht dieser Probleme wurden Nachforschungen betrieben, ob nicht eine einfachere und kostengünstigere Alternative zu den derzeit verfügbaren Funktionsanalysegeräten entwickelt werden kann, die eine gute Integration in den Klinikalltag gewährleistet. Nach einiger Recherche zu diesem Thema wurden Untersuchungen zu Bewegungsanalysen in anderen Bereichen der Medizin gefunden. Hier werden unter anderem Videoanaly- 
sen für die Messungen von Körperbewegungen verwendet. Im Zuge dieser Forschung werden an der Fakultät für Ingenieurwesen an der Universidad Nacional de Entre Rios (UNER) in Oro Verde, Paraná (Argentinien) seit längerer Zeit Analysen für die Bewegungen des Rumpfes sowie der oberen und unteren Extremitäten durchgeführt. Im Jahre 2007 veröffentlichte der Bioingenieur Dr. A. Braidot einen Artikel über die Bewegungsanalyse von Kniebeugen. Für diese Bewegungsmessungen wurden den Probanden lichtreflektierende Marker auf der Haut über den Gelenken angebracht und die Bewegungen mit Hilfe einer Videokamera aufgenommen. Die Auswertung erfolgte durch ein Softwareprogramm, welches am Institut der Universität entwickelt wurde. In diesem Programm werden die Marker via Tracking im Bild erkannt und verfolgt. Die Bewegungen werden automatisch als Graphen im Koordinatensystem dargestellt. Die Analysen sind sehr einfach und kostengünstig durchzuführen, da lediglich eine Kamera, Marker und ein bereits entwickeltes Analyseprogramm benötigt werden. Darüber hinaus ist der Zeitaufwand gering, da keine Geräte angebracht werden müssen. Außerdem kann die Methode den Bewegungsfluss nicht stören, da sie nichtinvasiv ist.

Die Bewegungen von Rumpf und Gliedmaßen bilden ähnliche Voraussetzungen für die Analyse wie die Bewegungen des Unterkiefers. Aus diesem Grund stellte sich die Frage, ob diese einfache, kostengünstige und nichtinvasive Methode der Bewegungsanalyse auch für den Einsatz in der Zahnmedizin geeignet sein kann.

Da diese Form der Videoanalyse bisher nur an Rumpf und Gliedmaßen Anwendung fand, wurde mit dem Einsatz im Bereich der Unterkieferbewegungsanalyse ein neues Forschungsfeld betreten. Insbesondere die Messgenauigkeit steht bei der Anwendung in der Zahnheilkunde im Vordergrund. In den bisherigen Analysen wurde in Zentimeter- und Dezimetergrößen gemessen, wohingegen sich die Unterkieferbewegungen im Millimeterbereich bewegen. Für die korrekte Berechnung muss also untersucht werden, wie die bestehenden Analyseverfahren auf die Messungen der Kieferbewegungen übertragen werden können.

Das angestrebte Analysesystem soll also nicht nur den Anspruch haben, kostengünstiger und einfacher in der Handhabung zu sein als die derzeit erhältlichen Geräte, sondern soll auch vergleichbare Messergebnisse liefern. Aus dieser Notwendigkeit wurde die Idee entwickelt, ein auf dem Markt etabliertes Funktionsanalysegerät als Vergleichsobjekt hinzuzu- 
ziehen. Dies hat den großen Vorteil, dass die Messgenauigkeit im direkten Vergleich überprüft werden kann. Darüber hinaus kann ein Vergleich des Zeitaufwandes und der Kosten angestellt werden.

Als Vergleichsgerät wurde der elektronische Gesichtsbogen ARCUS®digma der Firma KaVo ausgewählt. Dieses Gerät arbeitet mit Hilfe von Ultraschallsensoren, welche die Unterkieferbewegung im dreidimensionalen Raum registrieren und computerunterstützt aufzeichnen. Es werden Graphen für die Bewegung des Inzisalpunktes sowie der Kiefergelenke erstellt. Die einzelnen Bewegungen sind in Echtzeit auf einem Bildschirm sichtbar und Bewegungsmaxima werden automatisch errechnet.

Zur Ausstattung für die Videoanalyse gehören eine Videokamera, ein Computer ausgestattet mit entsprechender Analysesoftware und selbsthaftende Marker.

Da die meisten Bewegungsabweichungen in einer Ebene aufgezeichnet werden können, wurde entschieden, nur eine Kamera zu verwenden, wodurch auch die Kosten niedrig gehalten werden (Pinheiro et al. 2011).

Neben der Überprüfung der Messgenauigkeit soll in dieser Studie geprüft werden, ob die unterschiedliche Lokalisation der Messpunkte (intraoral und extraoral) zu divergierenden Messergebnissen führt und ob die Maxima-Werte der Bewegungen Aufschluss über eine Erkrankung des stomatognathen Systems geben. 


\section{Material und Methode}

\subsection{Genehmigung der Ethikkommission}

Diese Studie wurde von der Ethikkommission der Georg-August-Universität in Göttingen genehmigt (Dokumentennummer 5/6/13). Alle Probanden, die an der Studie teilgenommen haben, wurden über die Untersuchung aufgeklärt und unterzeichneten eine Einwilligungserklärung, die ebenfalls von der Kommission genehmigt wurde (siehe Anhang).

\subsection{Probanden}

Die Untersuchung wurde an 32 Probanden durchgeführt. Davon waren 15 weiblich und 17 männlich im Alter von 21 bis 57 Jahren $(27,2 \pm 6,8)$.

Bei der Probandenwahl waren die einzigen Ausschlusskriterien Zahnlosigkeit oder sehr wenige Zähne sowie festsitzende kieferorthopädische Apparaturen im Unterkiefer, da mit diesen Voraussetzungen der Unterkieferbogen des ARCUS®digma nicht an der Zahnreihe befestigt werden kann.

Alle Probanden zeichneten sich dadurch aus, dass subjektiv keine Funktionsstörungen vorhanden waren. Es wurden zuvor keine auffälligen Dyskoordinationen oder Limitationen festgestellt.

\subsection{Videografie}

Für die Aufnahmen wurden den Probanden farbige Marker auf das Kinn und die Nasenspitze beziehungsweise die Stirn geklebt und die Bewegungen des Unterkiefers mit Hilfe einer Sony-Videokamera (SONY, Handycam HDR-CX6EK) in HD-Qualität aufgenommen und im Sony-eigenen .MTS-Format gespeichert (Abb. 2).

Da die meisten Bewegungsabweichungen in einer Ebene aufgezeichnet werden können und Aufwand sowie Kosten gering gehalten werden sollten, wurde nur eine Kamera verwendet (Pinheiro et al. 2011).

Andere Studien zeigen, wie unterschiedlich die Kamerasysteme für die Videoanalyse aussehen können (Furtado et al. 2013, Braidot et al. 2007, Röhrle et al. 2009, Koseki et al. 
2007, Buschang et al. 2001). Dabei reicht der technische Aufwand von einfachen low-cost cameras bis hin zu high-end motion capture packs (Furtado et al. 2013).

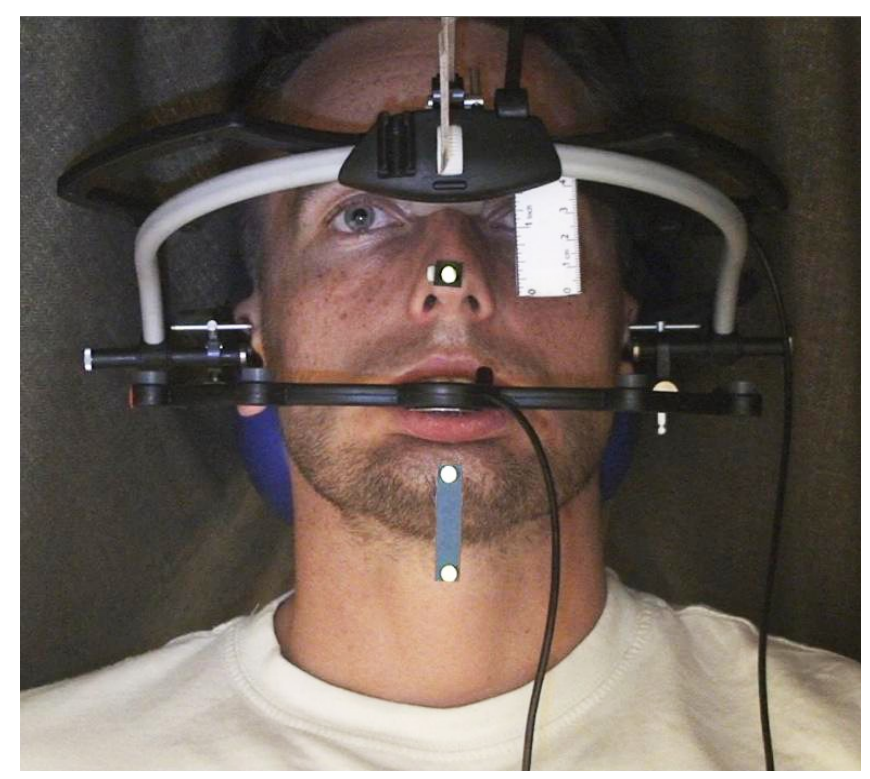

Abb. 2: Proband mit angelegtem Gesichtsbogen und aufgeklebten Markern.

\subsection{ARCUS $\circledast$ digma}

Der im Jahre 2007 von der Firma KaVo Dental eingeführte ARCUS®digma ist ein elektronischer Gesichtsbogen und steht der Abteilung Prothetik ZMK des Universitätsklinikums in Göttingen seit dem Jahr 2012 für die Patientenbehandlung zur Verfügung. Er wird zur instrumentellen Funktionsanalyse bei CMD Patienten eingesetzt und arbeitet berührungslos auf Ultraschallbasis (Schierz und Reißmann 2008).

Das Gerät besteht aus einem maxillären und einem mandibulären Bogen, einem Monitor zur Eingabe der Patientendaten und zur Ansicht der Bewegungsabläufe, einem Fußpedal, um die Bewegungsaufzeichnungen zu beginnen und zu beenden und einem paraokklusalen Löffel zur Befestigung des Unterkieferbogens.

Die beiden Bögen werden am Kopf des Patienten befestigt. Der obere Bogen (maxillärer Bogen) wird an der Nasenwurzel und den äußeren Gehörgängen sowie mit Hilfe eines elastischen Bandes über eine Druckbefestigung angebracht, der untere Bogen (mandibu- 
lärer Bogen) wird über einen paraokklusalen Löffel an den Zähnen des Unterkiefers fixiert. Durch die Anbringung des oberen Gesichtsbogens am Schädel und des Unterkieferbogens an der Unterkieferzahnreihe, sind beide Teile gegeneinander beweglich.

Das Gerät besitzt vier Ultraschallsender im unteren Bogen und acht Empfänger, die am oberen Bogen angebracht sind (Schmitter et al. 2011; Schierz und Reißmann 2008). Durch die Integration mehrerer Sender und Empfänger ist es möglich, die Bewegung des Unterkiefers im dreidimensionalen Raum aufzuzeichnen und Messfehler zu reduzieren (Schierz und Reißmann 2008).

Die oberen Ultraschallsensoren geben dabei die Kalibrierungsebene vor. Sie bilden eine feste Bezugsebene für den Unterkieferbogen. Die Bewegungen des Unterkiefers werden also in Abhängigkeit zu den oberen Sensoren registriert und berechnet. Dabei erfolgt sowohl die Bewegungsaufzeichnung des Inzisalpunktes als auch die der Kondylen.

Alle Bewegungsbahnen werden in Echtzeit auf dem Monitor des ARCUS $®$ digma als grafische Darstellung sichtbar (Schierz und Reißmann 2008) (Abb. 4). Das Programm liefert Graphen und eine tabellarische Auflistung der Maximalwerte, die nach Ablauf eines Bewegungsmusters am Computer abrufbar sind (Abb. 5). Darüber hinaus ist eine 3D-Ansicht der Bewegung möglich (Abb. 6).

Eine Studie aus dem Jahre 2007 zeigt, dass die Sensibilität des ARCUS®digma für das rechte Kiefergelenk bei 84,21 \% und für das linke Kiefergelenk bei 92,86 \% liegt. Die Spezifität liegt laut Studie für die rechte Kiefergelenkmessung bei 93,75 \% und für die Messung des linken Kiefergelenks bei 95,65 \%. Es wurde ein Konfidenzintervall von $95 \%$ für die Sensibilität und Spezifität errechnet. Das Ergebnis der Studie lautete, dass sich der ARCUS ${ }^{\circledR}$ digma als geeignetes Instrument für klinische Ergebnisse und Diagnosen von CMD-Erkrankungen eignet (Kobs et al. 2007). 


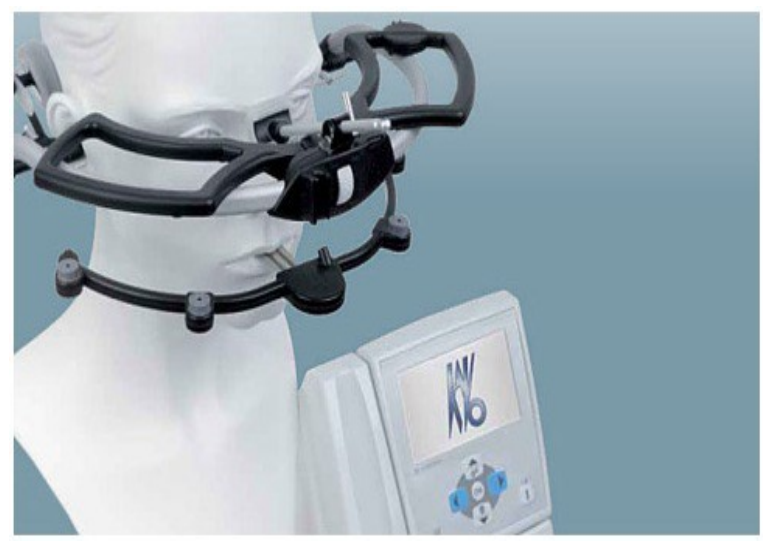

Abb. 3: ARCUS $®$ digma mit mobilem Monitor.

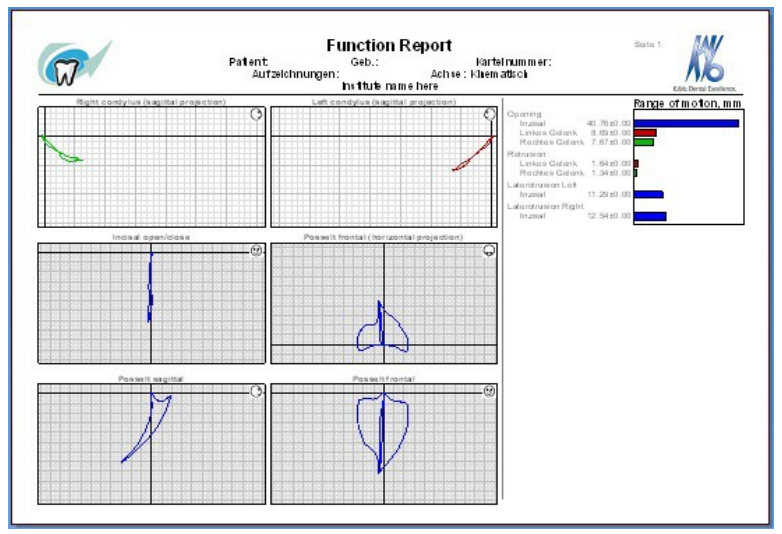

Abb. 5: Bewegungsgraphen und Liste der Maxima-Werte.

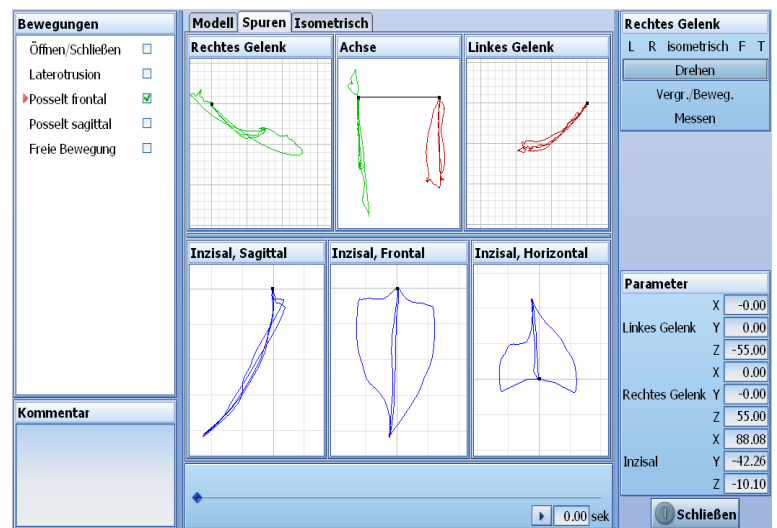

Abb. 4: Darstellung der Bewegungsbahnen im ARCUS $\otimes$ digma-Softwareprogramm.

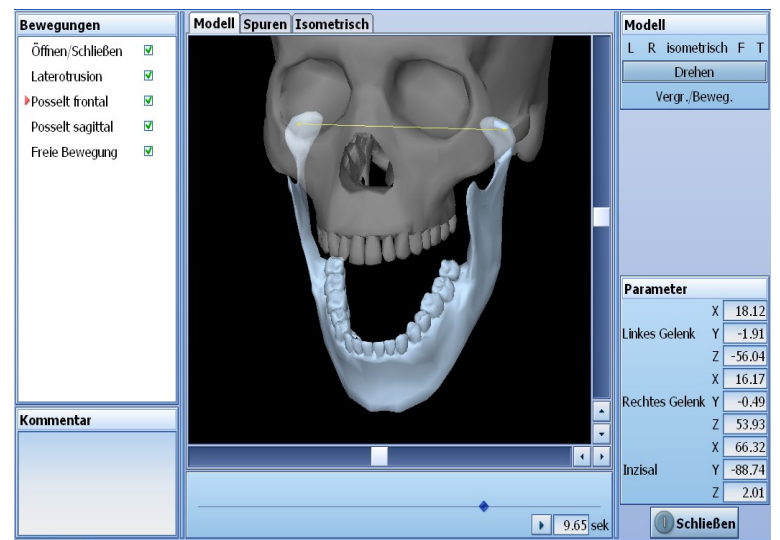

Abb. 6: Dreidimensionale Ansicht der Unterkieferbewegungen.

Quelle: http://selb.handrich.compedent.de/uploads/pics/Arcus-Digma.jpg

\subsection{Untersuchungsvorbereitung}

\subsubsection{Marker}

Das Softwareprogramm für die Videobewegungsanalyse benötigt einen hohen Kontrast zwischen Marker und umgebender Haut. Aus diesem Grund wurde für die Untersuchungsvorbereitung mit verschiedenen Markern und Lichteinstellungen gearbeitet, um das optimale Material und die beste Belichtung zu ermitteln. Zunächst wurden selbst-haftende Punkte in der Farbe neon-orange mit einem Durchmesser von $8 \mathrm{~mm}$, benutzt (Abb. 7, A), die aber nicht die gewünschten Ergebnisse lieferten. Um den Kontrast zu verstärken und 
die Größe des Kontrastfeldes im Softwareprogramm zu verringern, wurden Marker aus reflektierendem Material (gelb) und beidseitig klebendem Band hergestellt. Der Durchmesser dieser Marker betrug $5 \mathrm{~mm}$ (Abb. 7, B). Da die Belichtung nur eine schwache Reflexion zuließ, wurde der Raum zusätzlich abgedunkelt und eine Lichtquelle hinter der Videokamera angebracht, um die Reflektoren optimal zu belichten. Dieser Versuchsaufbau brachte das beste Ergebnis in Hinsicht auf die Kontrastgebung für die spätere Videoanalyse (Abb. 7, C).

Ein Marker wurde am Kinn fixiert, um die Unterkieferbewegung aufzuzeichnen. 1982 untersuchten Jemt und Hedegård, ob die Anbringung eines Markers auf der Kinnhaut zu Verfälschungen der Bewegung führt. Sie verglichen die Bewegungsbahnen des Kinnmarkers mit denen eines an den unteren Inzisiven angebrachten Markers und stellten fest, dass die Kinnreferenz für ihren untersuchten Kaurhythmus eingesetzt werden kann (Jemt und Hedegård 1982). Auch Kjellberg (Kjellberg et al. 1995), Jemt und Karlsson (Jemt und Karlsson 1980) benutzten den Kinnpunkt als Bewegungspunkt.

In anderen Studien hat sich gezeigt, dass es sinnvoll ist, einen weiteren Marker am Kopf zu befestigen, um die Kopfbewegungen später von den Unterkieferbewegungen abziehen zu können und somit die Messwerte für die reine Unterkieferbewegung zu erhalten (Pinheiro et al. 2011).

Atkinson und Shepherd arbeiteten schon 1955 mit Markern, die auf Kinn und Nasenspitze befestigt waren (Atkinson und Shepherd 1955). Auch Karlsson arbeitete 1977 mit zwei Licht-emittierenden Dioden. Eine Bewegungsdiode wurde am Kinn und eine Referenzdiode an der Stirn befestigt (Karlsson 1977).

Da sich diese Hautareale zur Markerbefestigung in vorangegangenen Studien bewährt haben und eine geringe Beweglichkeit des Weichgewebes über dem Knochen zeigen, wurde auch in der vorliegenden Studie ein Marker auf dem Kinn und ein zweiter Marker auf der Nasenspitze oder der Stirn befestigt. 

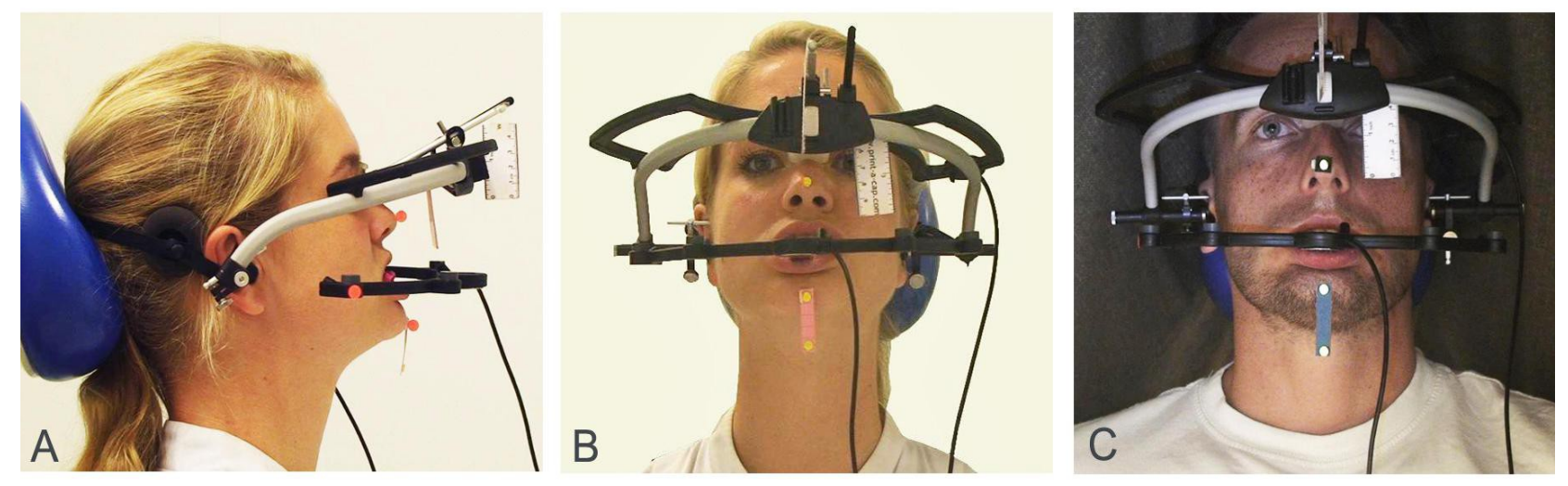

Abb. 7: A: Marker in neon-orange mit einem Durchmesser von $8 \mathrm{~mm}$; B: Marker aus reflektierendem Material (gelb) mit einem Durchmesser von $5 \mathrm{~mm}$; C: Reflektierender Marker im abgedunkelten Raum.

\subsubsection{Skalierung}

Die Umrechnung der im Video sichtbaren Bewegungen in Graphen mit korrekten Millimeterangaben stellte sich zunächst als grundlegendes Problem dar.

Die Bildauflösung der Videoaufnahmen wird in Pixeln gemessen. Jedes Pixel hat dabei eine bestimmte Kantenlänge, die aber keinen Schluss auf die Größe des abgebildeten Objektes zulässt. Je nach Abstand der Kamera und der Kameraeinstellung selbst, variiert die abgebildete Größe eines Objektes oder einer Person.

Um bei der späteren Auswertung der Videoaufnahmen die Möglichkeit der Umrechnung von Pixeln in $\mathrm{mm}$ zu haben, musste im Video eine Skalierung angebracht werden. In der vorliegenden Untersuchung wurden Millimetermaße verwendet, die in der Ebene der jeweils auszuführenden Bewegung angebracht wurden (auf Marker-Ebene) und somit während des gesamten Bewegungsablaufs sichtbar waren. Bei der frontalen Grenzbewegung wurde die Skala auf Höhe des Kinnmarkers angebracht und in frontaler Ausrichtung befestigt. Bei der Posselt-sagittal-Bewegung wurde die Skala in der Medianebene fixiert und so ausgerichtet, dass sie von sagittal lesbar war.

Mit Hilfe dieses Versuchsaufbaus war es möglich, für jede Bewegung eine individuelle Pixel-Millimeter-Umrechnung durchzuführen. 


\subsubsection{Auswahl der Bewegung}

Abgesehen von der Markerpositionierung und der korrekten Skalierung der Bewegungsbahnen, ist die Auswahl der Bewegungen von zentraler Bedeutung für den Vergleich der Analysemethoden. Für die Untersuchungen war es am sinnvollsten, den gesamten Spielraum der Unterkieferbewegung zu nutzen und den Fokus auf die Bewegungsmaxima zu legen. Nur auf diese Weise kann man reproduzierbare Ergebnisse erhalten, die auch für einen dokumentierenden Vergleich eingesetzt werden können. Darüber hinaus ist davon auszugehen, dass bei größeren Bewegungen, mögliche Messfehler weniger stark ins Gewicht fallen.

Die Maximalstellungen des Unterkiefers werden auch als Grenzpositionen bezeichnet. (Strub et al. 2011). Diese Kieferstellungen sind durch ihre klare Begrenzung reproduzierbar und geben den Rahmen vor, innerhalb dessen alle funktionellen Bewegungen des Unterkiefers ablaufen (Lehmann et al. 2009).

Um die Studienergebnisse vergleichen zu können, sollte jeder Proband die gleichen Bewegungen durchführen. Die Entscheidung fiel dabei auf die Grenzbewegungen in der Frontalebene (Abb. 8) sowie das von Posselt entwickelte Grenzbewegungsmuster in der Sagittalen („Posselt sagittal“) (Lehmann et al. 2009) (Abb. 9). Es handelt sich dabei um klassische Kurven, um die Maximalbewegungen des Unterkiefers zu erfassen.

Der Bewegungsablauf der frontalen Kurve gliedert sich in die zahngeführte Bewegung des Unterkiefers zur einen Seite (Laterotrusion), die maximale Mundöffnung aus dieser Position und den Kieferschluss. Daraufhin wird dieser Bewegungsablauf für die entgegengesetzte Seite durchgeführt (Abb. 10).

Die Posselt-sagittal-Kurve beschreibt den maximalen Vorschub des Unterkiefers unter Zahnkontakt (Protrusion), die maximale Öffnung aus dieser Position und den Kieferschluss (Abb. 11). Es handelt sich bei den Bewegungen um kombinierte Bewegungen bestehend aus dynamischer (unter Zahnkontakt) und freier (ohne Zahnkontakt) Unterkieferbewegung (Lehmann et al. 2009).

Da die Graphen aus Maximal- beziehungsweise Grenzbewegungen bestehen, sind sie reproduzierbar. Sie beinhalten außerdem alle Bewegungen, die sich sowohl mit dem ARCUS®digma als auch mit der Videoanalyse aufzeichnen lassen.

Anhand von Form, Länge und Symmetrie der aufgezeichneten Graphen kann indirekt auf 
zugrunde liegende Krankheitsbilder geschlossen werden (Schierz und Reißmann 2008). Auch Pinheiro, Fang und Kuo nahmen die Bewegungen Öffnen-Schließen, Protrusion und Laterotrusion als Grundlage ihrer Untersuchungen, da diese speziellen Bewegungen aus ihrer Sicht für die funktionelle Evaluation des Unterkiefers grundlegend sind (Fang und Kuo 2008; Pinheiro et al. 2011). In weiteren Studien wurde die reine Mundöffnung als Analysegrundlage verwendet (Sadat-Khonsari et al. 2003; Travers et al. 2000; Yoon et al. 2006).

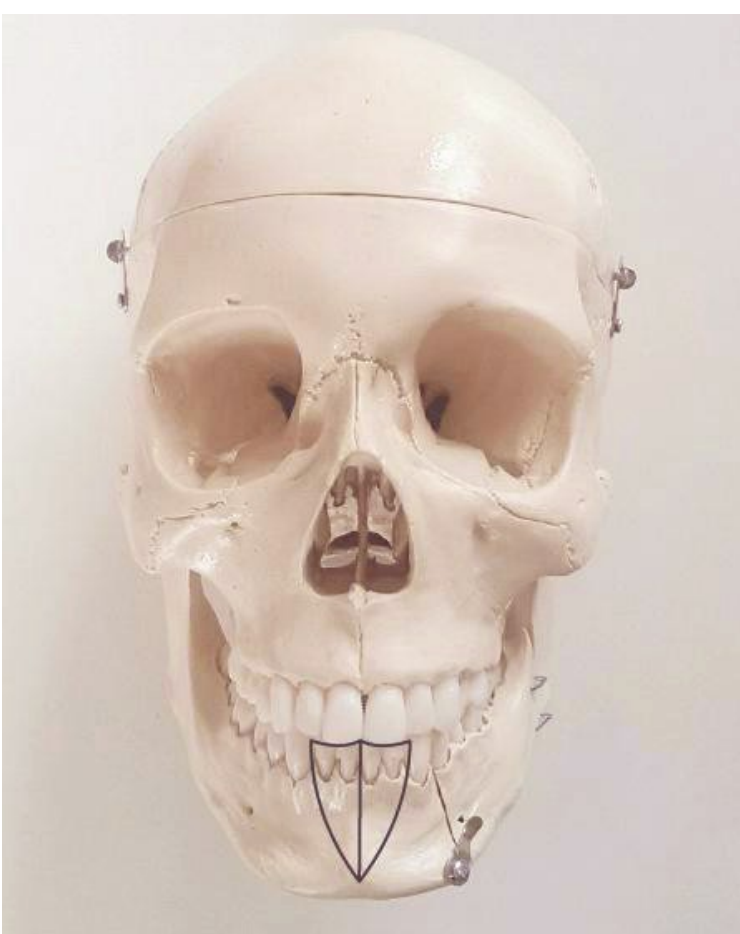

Abb. 8: Grenzbewegungen in der Frontalebene.

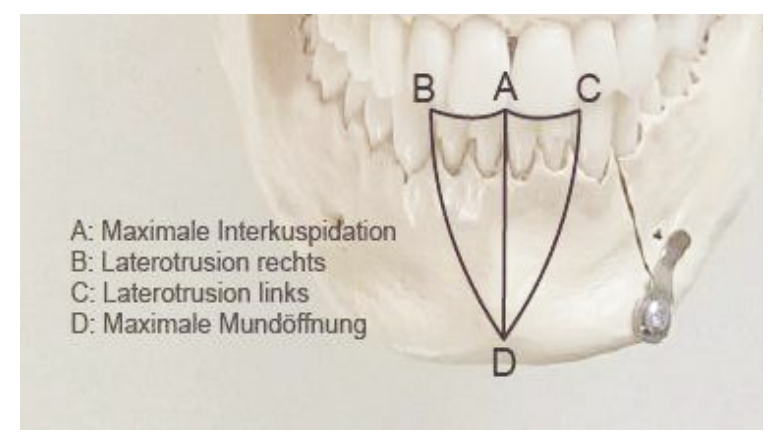

Abb. 10: Bewegungsablauf der frontalen Grenzbewegung.

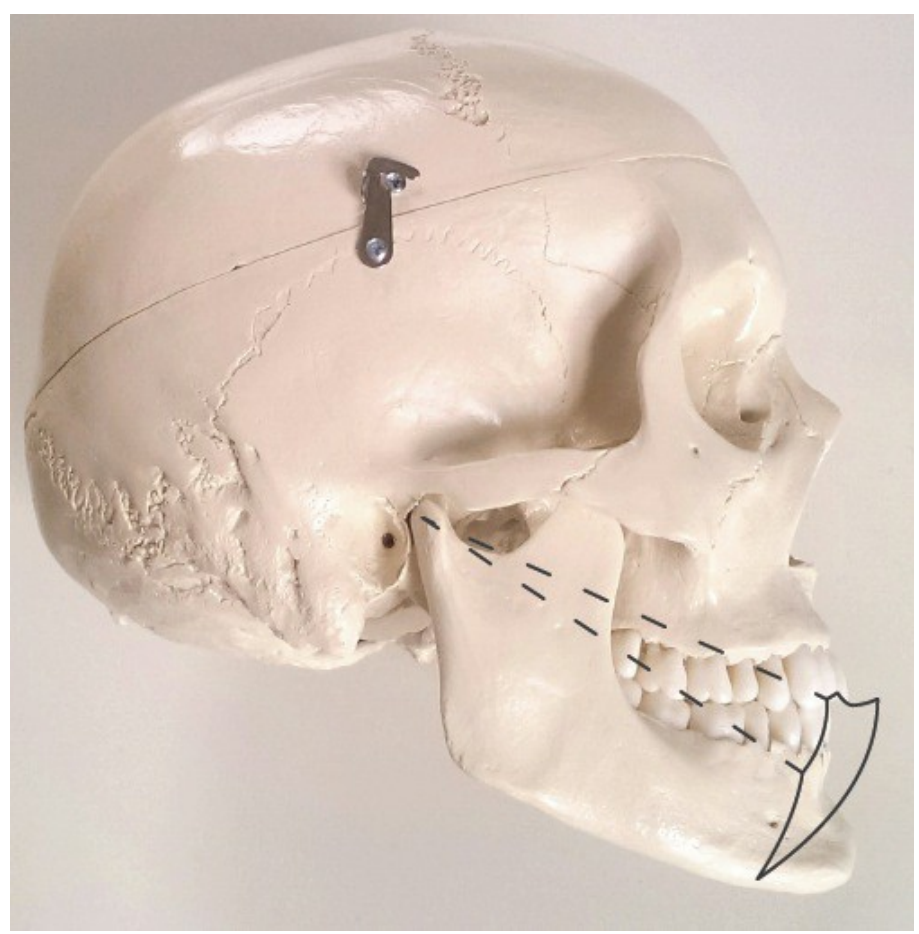

Abb. 9: Grenzbewegungen in der Sagittalebene („Posselt sagittal").

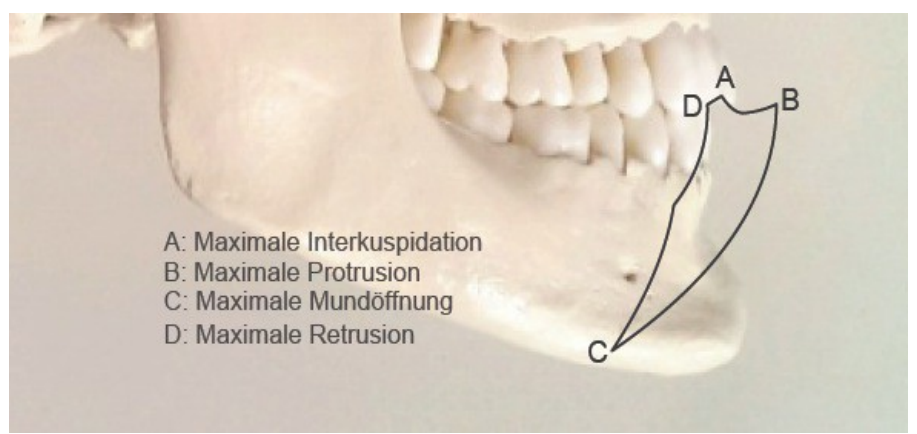

Abb. 11: Bewegungsablauf der sagittalen Grenzbewegung. 


\subsection{Untersuchungsdurchführung}

\subsubsection{Anlegen des ARCUS $囚$ digma}

Für die Versuchsdurchführung wurde den Probanden zunächst der elektronische Gesichtsbogen angelegt (Abb. 12, A-G). Dafür wurde der obere Teil des ARCUS®digma mit Hilfe von Stellschrauben und einem elastischen Band am Kopf befestigt. Wichtige Fixpunkte sind hierbei die Nasenwurzel und die äußeren Gehörgänge (Abb. 12, A/B). Der Unterkieferbogen wurde mit Hilfe eines paraokklusalen Löffels an den Unterkieferzähnen fixiert. Zur Befestigung des Löffels wurde das A-Silikon FutarD® der Firma Kettenbach verwendet (Abb. 12, C-F).

Nach der Befestigung des Gesichtsbogens sollte der Proband die Zähne in Interkuspidation (Zahnkontakt) bringen. Über die Ultraschallsensoren wurde die Lage des Unterkieferbogens in Bezug zum Gesichtsbogen kalibriert (Abb. 12, F). Von dieser Nullpunktlage ausgehend, wurden alle folgenden Bewegungsvektoren automatisch errechnet.

Jede Bewegung wird auf dem Monitor des ARCUS®digma als grafische Darstellung sichtbar und ist nach Ablauf eines Bewegungsmusters am Computer abrufbar (Abb. 12, H). Das Programm liefert sofort Graphen und Maximalwerte. Darüber hinaus ist eine 3D-Ansicht der Bewegungen möglich. 


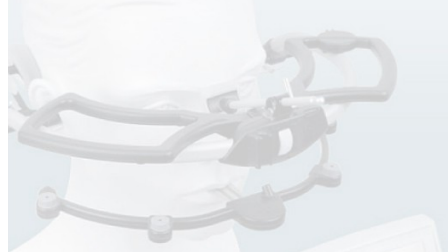

Anlegen des

elektronischen

Gesichtsbogens
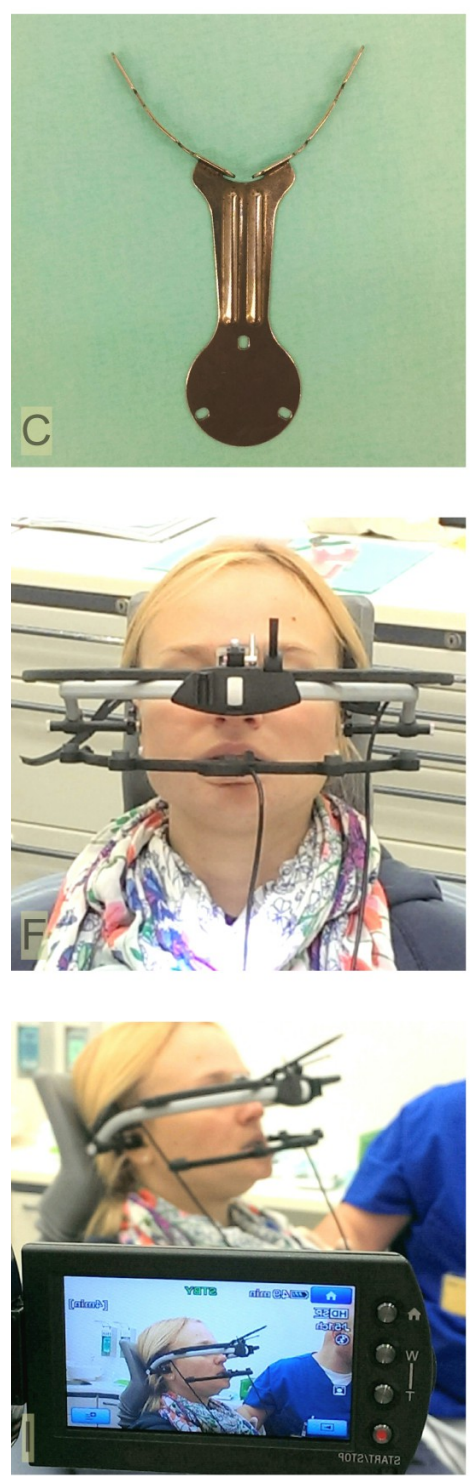
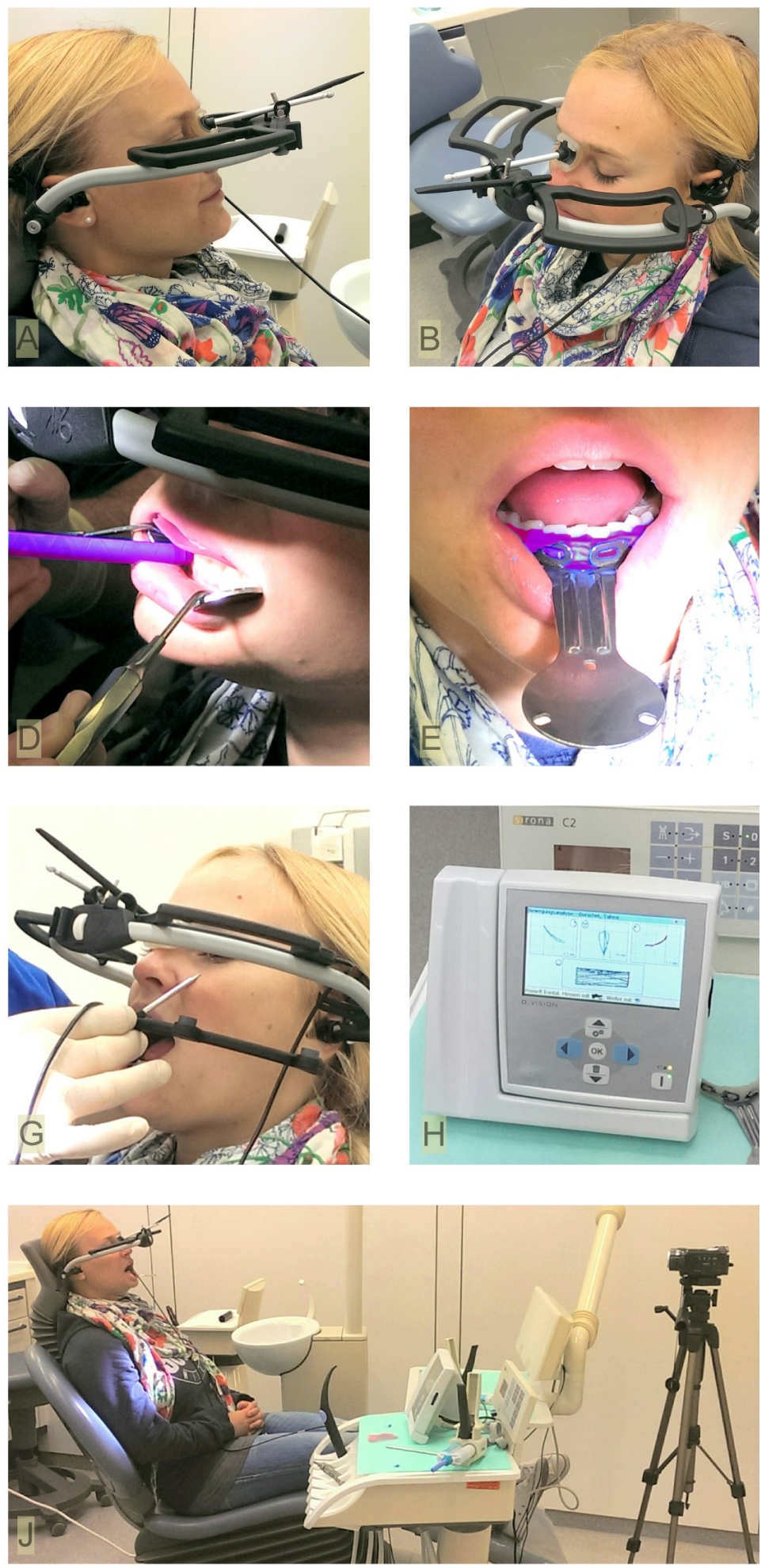

Abb. 12: Anlegen des ARCUS $₫$ digma: $A$ und B: Angelegter Oberkieferbogen; C: Paraokklusaler Löffel; D: Fixierung einer Wachsplatte zwischen den Zähnen und Einbringen des Befestigungsmaterials FutarD®; E: Fixierung des paraokklusalen Löffels; F: Angelegter Unterkieferbogen und Kalibrierung; G: Registrierung des Foramen infraorbitale; H: Darstellung der Bewegungskurven auf dem ARCUS $®$ digma-Monitor; I: Videoaufnahme während der sagittalen Bewegungsaufnahme; J: Versuchsaufbau für die frontale Bewegungsaufnahme. 


\subsubsection{Anbringen der Marker}

Nach dem Anlegen des Gesichtsbogens wurden den Probanden die Marker auf dem Kinn und auf der Nasenspitze beziehungsweise der Stirn angebracht. Die Videokamera wurde so ausgerichtet, dass die Marker von frontal gut zu erkennen waren und diese die Bildfläche auch bei Bewegungen nicht verlassen würden (Abb. 13).

Für die sagittalen Bewegungen (Posselt sagittal) wurde den Markern auf Nasenspitze und Kinn jeweils ein Marker im rechten Winkel hinzugefügt, sodass diese von sagittal aus gut zu sehen waren. Für die Videoaufnahmen von sagittal wurde die Kamera umgestellt und die Bewegungen von der Seite her aufgenommen (Abb. 14).

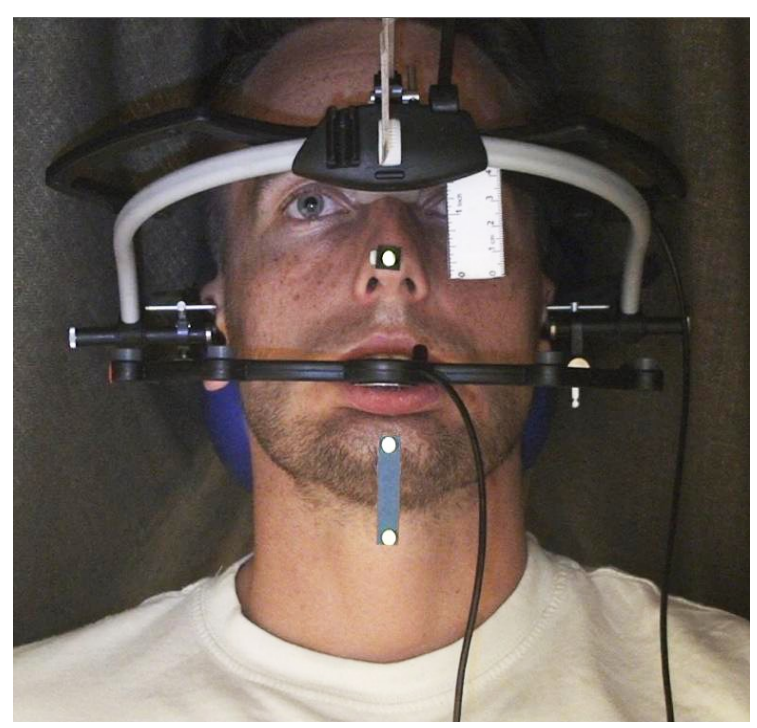

Abb. 13: Kameraaufnahme von frontal mit angebrachten Markern.

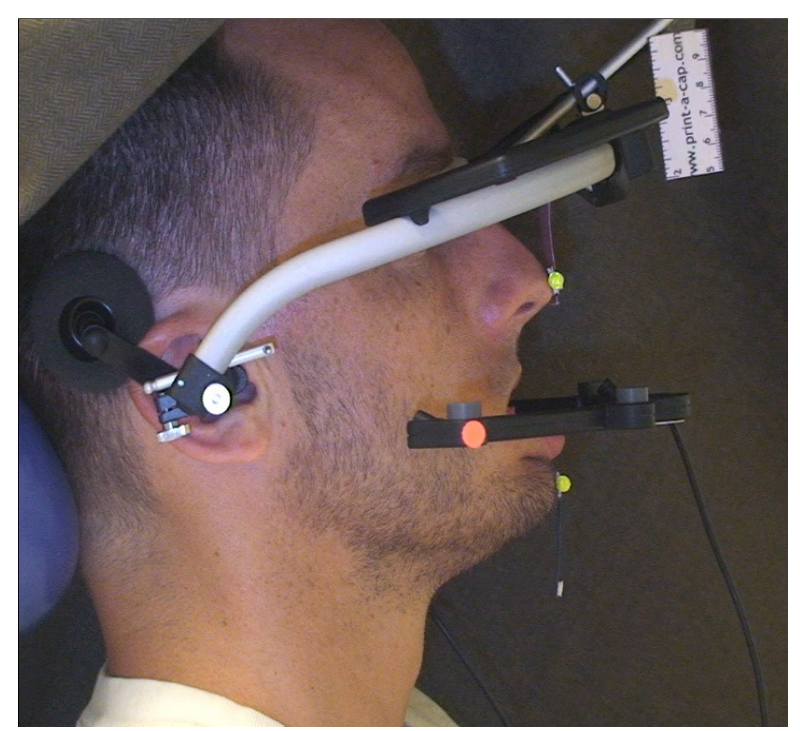

Abb. 14: Kameraaufnahme von sagittal mit angebrachten Markern.

\subsubsection{Bewegungsablauf}

Nach dem Anlegen des ARCUS®digma und Anbringen der Marker wurden den Probanden die durchzuführenden Bewegungen erklärt und gemeinsam geübt. Daraufhin wurde jede Person gebeten, sich aufrecht auf den Behandlungsstuhl zu setzen. Der Kopf sollte dabei immer ruhig gehalten werden und zur Kamera ausgerichtet sein. Die Kamera befand sich vor dem Behandlungsstuhl im Abstand von 1,5 m zu den Markern (Abb. 12, J).

Nach der Kalibrierung des Gesichtsbogens wurden zunächst die frontalen Grenzbewegungen aufgezeichnet. Anschließend wurde die Kamera seitlich des Stuhls platziert, um die 
Sagittalebene aufnehmen zu können (Abb. 12, I). Daraufhin konnte die Posselt-sagittalBewegung durchgeführt und aufgenommen werden. Dieser Ablauf wurde für jeden Probanden fünf Mal wiederholt.

Während der Bewegungsdurchführung wurden den Probanden die jeweiligen Grenzbewegungen sowie die einzelnen Bewegungsabschnitte angesagt, um Bewegungsfehler zu minimieren.

\subsection{Verarbeitung der Messdaten}

\subsubsection{Konvertieren in .AVI}

Die Videoaufnahmen wurden mit einer Sony-Videokamera (SONY, Handycam HDRCX6EK) in HD-Qualität aufgenommen und im Sony-eigenen .MTS-Format gespeichert.

Da das Programm Rastreobackup, welches zur Analyse des Bildmaterials eingesetzt wurde, dieses Filmformat nicht unterstützt, wurden die Aufnahmen in das Format .AVI konvertiert. Das Konvertieren geschah mit Hilfe des Programms AVS Video Editor.

Durch die Formatänderung wurde die Qualität der Aufnahmen reduziert. Des Weiteren nahm das Umschreiben der Videos viel Zeit in Anspruch. Dafür erlaubt das Programm AVS Video Editor dem Nutzer aber auch die Filmaufnahmen zuzuschneiden, zu zentrieren und einzuzoomen. Dies wurde für alle Videosequenzen durchgeführt, um störende Bewegungen vor und nach der eigentlichen Bewegungsanalyse auszuschließen und die Marker deutlicher zu fokussieren.

\subsubsection{Rastreobackup}

Nachdem die Filmaufnahmen im .AVI-Format vorlagen, wurde jedes Video in dem Programm Rastreobackup geöffnet.

Das Programm wurde im biomechanischen Labor der Fakultät für Ingenieurwesen an der Universidad Nacional de Entre Rios (UNER) in Oro Verde, Paraná (Argentinien) für Bewegungsanalysen entwickelt. Man kann mit dieser Software einen Punkt im Bild markieren, welcher daraufhin im Verlauf des Videos immer wiedererkannt und verfolgt wird (Tracking). 
In der vorliegenden Studie wurden die Mittelpunkte der Marker an Stirn/Nase und Kinn als zu registrierende Objekte mit einem Fadenkreuz gekennzeichnet.

$\mathrm{Zu}$ jedem Zeitpunkt der Aufnahme befanden sich die Marker an einem anderen Ort und wurden von der Software immer neu lokalisiert. Somit unterteilt das Programm den Bewegungsablauf in viele Einzelbilder und ordnet dem jeweiligen Ort des Markers den zugehörigen Ortsvektor im Koordinatensystem zu. Durch die Verbindung dieser Einzelpunkte, lässt sich die Bewegung als Graph darstellen.

Um die Graphen der Grenzbewegungen aus den Videos später mit den Kurven des ARCUS $®$ digma-Programms vergleichen zu können, mussten sie in ihrer Ausrichtung und Bemaßung an die Gesichtsbogendaten angepasst werden.

Für die Bemaßungsanpassung wurde während der Videoaufnahme ein Lineal mit Millimetereinteilung in der Ebene der Marker (frontale Grenzbewegung: Nasenspitze und Kinn; Posselt sagittal: Medianebene) angebracht. Anhand dieser Skalierung konnten die Pixel der Videoaufnahmen in Millimeter umgerechnet werden. Als Grundlage für die Berechnungen wurden Punkte auf der Millimeterskala in der Software Rastreobackup ausgewählt, mit C1, C2, C3 und C4 beschriftet und die Abstände dieser Punkte im Programm in Millimetern angegeben (Abb. 15).

Der Ausschnitt eines auf einem Pixel abgebildeten Objektes hängt vom Abstand der Kamera zum Aufnahmeobjekt ab. Aus diesem Grund musste diese Berechnung für jedes Video separat durchgeführt werden.

Da die Ausrichtung der Videoaufnahmen nicht der der ARCUS®digma-Vermessung entspricht, mussten die Filmaufnahmen darüber hinaus der Gesichtsbogen-Ebene angepasst werden. Zur korrekten Ausrichtung der Filmsequenzen wurde die obere Sensorenebene des ARCUS $®$ digma herangezogen. Diese Ebene dient dem Gesichtsbogen als Kalibrierungsebene und liegt dem Kopf des Probanden fest an. In der vorliegenden Untersuchung wurde die Ebene als Linie angenommen, da auch die entstehenden Graphen nur im zweidimensionalen Raum (Frontalebene) abgebildet werden. Für die Registrierung der Sensoren- und Kalibrierungsebene im Programm Rastreobackup wurden die Endpunkte des Stirnbogens mit AD1 und AD2 markiert (Abb. 15). Die Verbindungslinie zwischen diesen Punkten wurde horizontal ausgerichtet und die Graphen aus der Videoanalyse somit in die korrekte Position geschwenkt. 


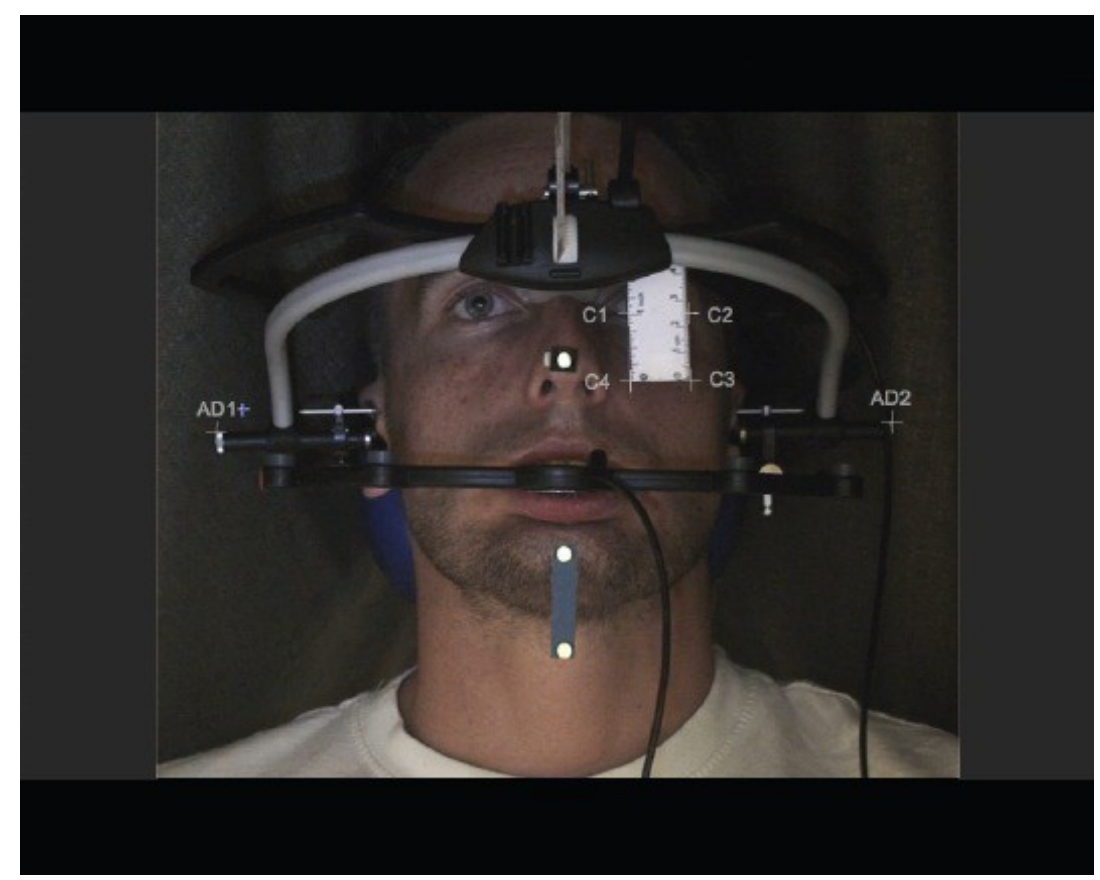

Abb. 15: Frontale Videoaufnahme mit den Beschriftungen der Millimeterskala (C1-4) und der Markierung der Endpunkte des Stirnbogens (AD1 und AD2).

\subsubsection{MATLAB® (MATrix LABoratory)}

Um die Kurven der Videomessung und des Gesichtsbogens vergleichen zu können, wurden alle Graphen in das Programm MATLAB® überschrieben. Dabei handelt es sich um eine Computer- und Programmiersoftware der Firma The MathWorks (Natick, Massachusetts (USA)). Das Programm wird zur Lösung mathematischer Probleme und für die grafische Ergebnisdarstellung eingesetzt. Die numerischen Berechnungen werden mit Hilfe von Matrizen durchgeführt. Von diesem Systemaufbau leitet sich auch der Name des Programms ab.

Die Video-Daten des Programms Rastreobackup waren für die MATLAB®-Software direkt kompatibel. Die Daten des ARCUS®digma mussten zunächst aus dem Gesichtsbogenprogramm exportiert werden. 


\subsubsection{Export der ARCUS $®$ digma-Daten}

Um die Daten des elektronischen Gesichtsbogens in dem Programm MATLAB® öffnen zu können, wurden die Messwerte aus der ARCUS®digma-Software exportiert (.txt-Format). Daraufhin wurden die Daten aus dem Unicode UTF16 in den Unicode UTF8 transferiert, um von $M A T L A B \otimes$ gelesen werden zu können und alle Graphen in das Programm MAT$\angle A B \circledast$ überschrieben.

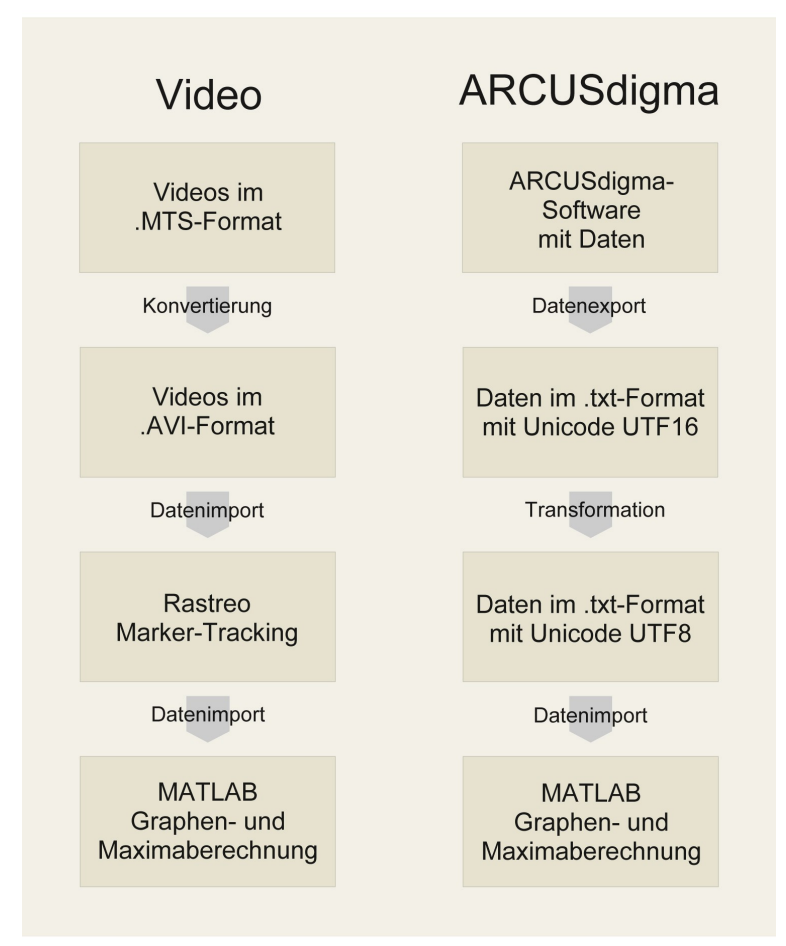

Abb. 16: Grafische Darstellung des Ablaufs der Datentransformation. 


\subsection{Maximaberechnung}

\subsubsection{Auswahl der Werte}

Um die Graphen des elektronischen Gesichtsbogens mit den Graphen der Videoanalyse vergleichen zu können, mussten zunächst Vergleichsparameter der Bewegungen ausgewählt werden. Da der Bewegungsablauf aus Grenzbewegungen besteht, wurden die Maximalwerte dieser Bewegungen als Vergleichsgrundlage verwendet. Für die frontale Grenzbewegung sind dies die maximalen Werte aus der Laterotrusion nach rechts, der Laterotrusion nach links sowie der Mundöffnung (Abb. 17). Für den Bewegungsablauf Posselt sagittal wurde die maximale Protrusion sowie die maximale Mundöffnung berechnet (Abb. 18).

In der Software des ARCUS $R$ digma werden die Maximalwerte automatisch ermittelt und in einer patientenbezogenen Tabelle angezeigt. Die Berechnung erfolgt dabei über eine Gerade. Es werden Ausgangspunkt und Maximalpunkt einer Bewegung registriert und eine Verbindungslinie zwischen beiden Punkten gezogen. Unregelmäßigkeiten in der Bewegung werden nicht mit in die Berechnung einbezogen.

Da die Gesichtsbogensoftware auf diese Art arbeitet und eine Studie von Buschang et al. ergeben hat, dass sich eine Gerade zur Messung und für den Vergleich der Maximalwerte besser eignet, als eine dem Bewegungsbogen folgende Kurve (Buschang et al. 2001), wurden auch die Maximalwerte der Videoanalyse auf die gleiche Weise ermittelt.

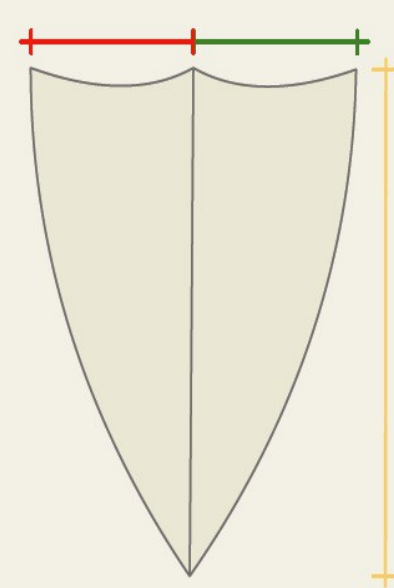

- Laterotrusion rechts

- Laterotrusion links

Maximale Mundöffnung

Abb. 17: Maxima-Werte für die frontale Grenzbewegung.

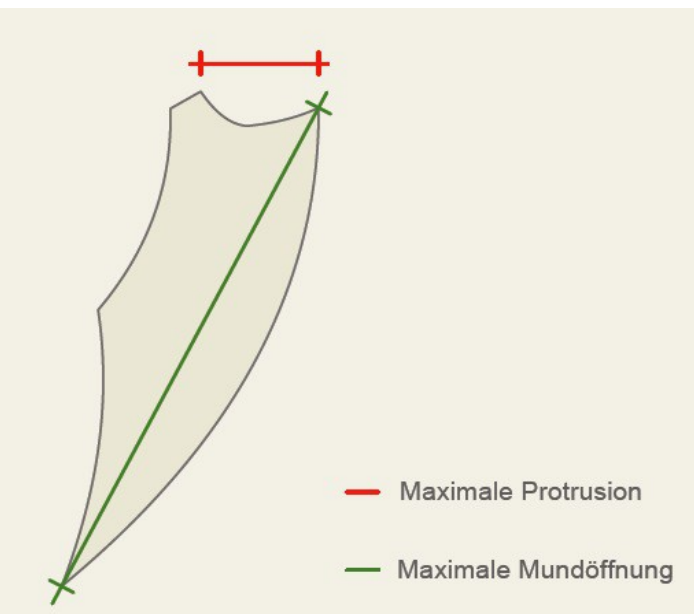

Abb. 18: Maxima-Werte für die sagittale Grenzbewegung. 


\subsubsection{Maxima Videoanalyse - frontale Grenzbewegung}

Alle Graphen wurden im Programm MATLAB® (Matrix Laboratory) geöffnet und die gesuchten Maxima mit Hilfe der Software ermittelt. Es wurden dabei die Bewegungsmaxima für die Laterotrusionsbewegungen nach rechts und links sowie für die Mundöffnung berechnet (Abb. 19).

Bei der Videoanalyse handelt es sich um eine zweidimensionale Projektion. Aus diesem Grund konnten die Messungen der Grenzbewegungen nur in der Frontalebene vorgenommen werden. Da die Mundöffnung vorwiegend in der Sagittalebene abläuft, führt die Projektion der Bewegung auf die frontale Ebene zur Stauchung des Graphen. Um eine einheitliche Vergleichsgrundlage zu haben, wurden für die ARCUS®digma-Werte ebenfalls die Projektionswerte der Frontalebene verwendet.

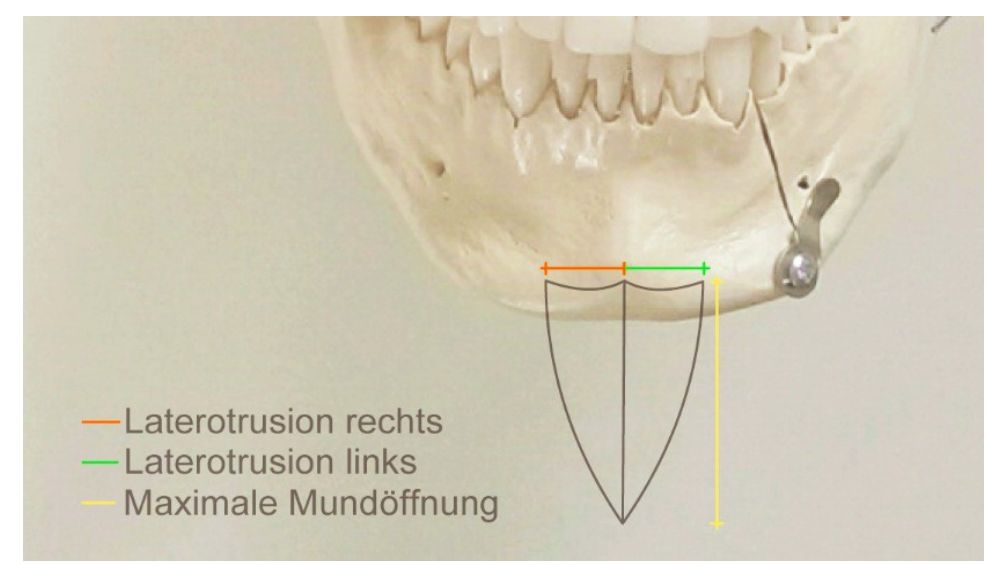

Abb. 19: Frontale Grenzbewegung ausgehend vom Kinnpunkt.

\subsubsection{Maxima Videoanalyse - Posselt sagittal}

Alle Graphen wurden im Programm MATLAB® (Matrix Laboratory) geöffnet und die gesuchten Maxima für die Protrusion und die Mundöffnung mit Hilfe der Software berechnet. Die Posselt-sagittal-Bewegung erfolgt maßgeblich in der Sagittalebene, welche auch die Aufnahmeebene darstellt. Aus diesem Grund kam es zu keiner Projektionsverkürzung der Graphen (Abb. 20). 


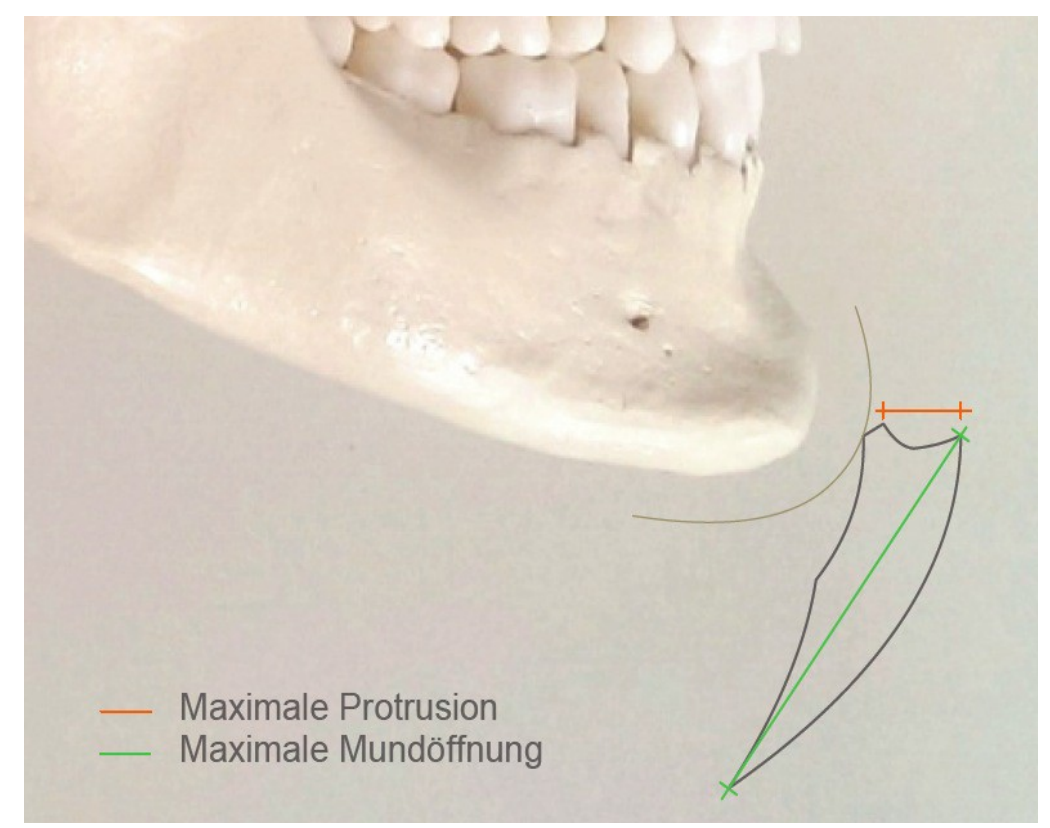

Abb. 20: Sagittale Grenzbewegung ausgehend vom Kinnpunkt.

\subsubsection{Maxima ARCUS $\circledast$ digma - frontale Grenzbewegung}

Die Maxima für die Bewegungen Laterotrusion rechts und Laterotrusion links werden im ARCUS ${ }^{\circledR}$ digma-eigenen Programm automatisch errechnet. Sie sind in einer patientenbezogenen Tabelle ablesbar.

Die Mundöffnungsbewegung erfolgt maßgeblich in der sagittalen Ebene. Da die Videoanalyse für die frontalen Grenzbewegungen aber nur in der Frontalebene arbeitet, wurde auch

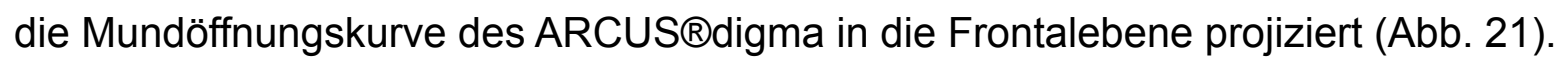

Für die Berechnung der Öffnungsbewegung in dieser Ebene wurde mit Hilfe der ARCUS ${ }^{2}$ digma-Software eine Senkrechte gezeichnet und anhand dieser die Länge der Grenzbewegungen bestimmt. Abweichungen nach rechts oder links wurden vernachlässigt. 


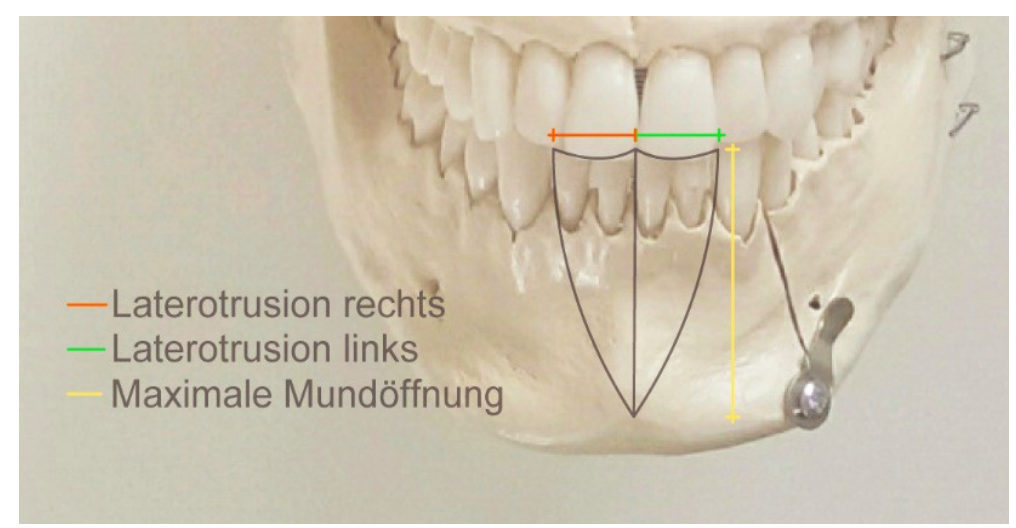

Abb. 21: Frontale Grenzbewegung ausgehend vom Inzisalpunkt.

\subsubsection{Maxima ARCUS $\circledast$ digma - Posselt sagittal}

Die maximale Mundöffnung wird in der ARCUS $\AA$ digma-Software berechnet und ist in einer Tabelle ablesbar.

Da die Maximalwerte für die Protrusionsbewegung nicht angegeben werden, wurden diese in den Graphen abgemessen. Dafür wurden Start- und Endpunkt der Bewegung miteinander verbunden und die Länge der Verbindungslinie ermittelt.

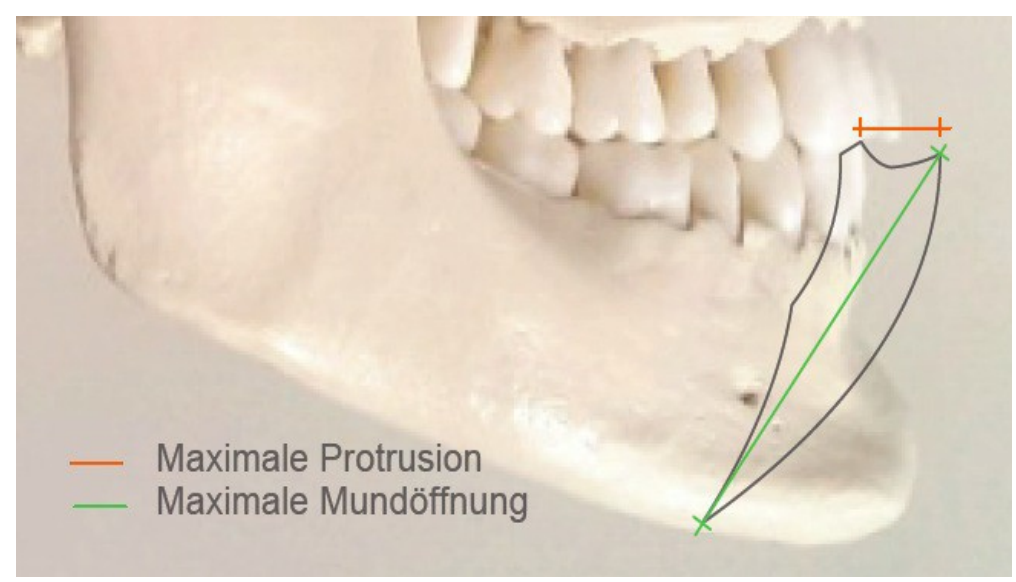

Abb. 22: Sagittale Grenzbewegung ausgehend vom Inzisalpunkt. 
Die Berechnungen der Daten in dem Programm MATLAB® wurden in Zusammenarbeit mit dem Institut für Bioingenieurwesen an der Universität Entre Rios in Oro Verde, Paraná (Argentinien) durchgeführt.

\subsubsection{Vergleichsberechnung}

Beim Vergleich der Kurven fällt auf, dass die Graphen der Videografie tendenziell größer sind als die des Gesichtsbogens. Dies ließ die Frage nach der Ursache dieser regelmäßigen Abweichung aufkommen. Nach Betrachtung der verschiedenen Einflussfaktoren wurde deutlich, dass die Größendifferenz der Graphen auf die verschiedenen Messpunkte zurückzuführen ist (Inzisalpunkt und Kinnpunkt).

Um die Vermutung zu überprüfen, wurden Berechnungen durchgeführt und die theoretischen Bewegungen des Unterkiefers anhand von Grafiken analysiert. Zunächst wurde dafür angenommen, dass die durchschnittliche Distanz der Messpunkte Inzisalpunkt und Kinnpunkt in der Sagittalebene $2 \mathrm{~cm}$ beträgt. Aus den Laterotrusionswerten des ARCUS®digma wurde ein Mittelwert von 10,067 mm errechnet. Mit Hilfe dieses Wertes und einer Sin-/Cos-Berechnung ${ }^{1}$ erhält man den Wert, der bei einem Abstand der Messpunkte von $2 \mathrm{~cm}$ dem durchschnittlichen Laterotrusionswert der Videografie entsprechen müsste (Abb. 23). Bei der Berechnung ergab sich ein Wert von 12,29 mm. Der durch die Videomessungen errechnete Mittelwert beträgt aber 13,249 mm. Daraufhin wurde die tatsächlich durchschnittliche Distanz der Messpunkte berechnet. Diese beträgt rund $3 \mathrm{~cm}$ (Abb. 24).

Anhand der Mittelwerte des ARCUS®digma und der Videografie können darüber hinaus Umrechnungsfaktoren bestimmt werden. Mit Hilfe dieser Faktoren wurde eine theoretische Maximaberechnung für die Bewegung Laterotrusion links (Faktor: 1,754), Laterotrusion rechts (Faktor: 1,754) und sagittale Mundöffnung (Faktor: 1,096) durchgeführt.

1.: C (Tabellenwert Maximum ARCUS®digma) * $\operatorname{Sin}\left(90^{\circ}\right) / \operatorname{Sin}\left(59^{\circ}\right)=A$

2.: $\operatorname{ACos}^{*}((A * A-10 * 10-10 * 10) /(-2 * 10 * 10)=$ Winkela

3.: $\sqrt{ }\left(\left(117,7^{*} 117,7\right)+\left(117,7^{*} 117,7\right)-\left(2^{*} 117,7^{*} 117,7^{*} \operatorname{Cos} \alpha\right)\right)=c$

4.: $c^{*} \operatorname{Sin}\left(63,5^{\circ}\right) / \operatorname{Sin}\left(90^{\circ}\right)=$ theoretisches Ergebnis $(\rightarrow$ Vergleich mit tatsächlich ermittelten Tabellenwerten $)$ 


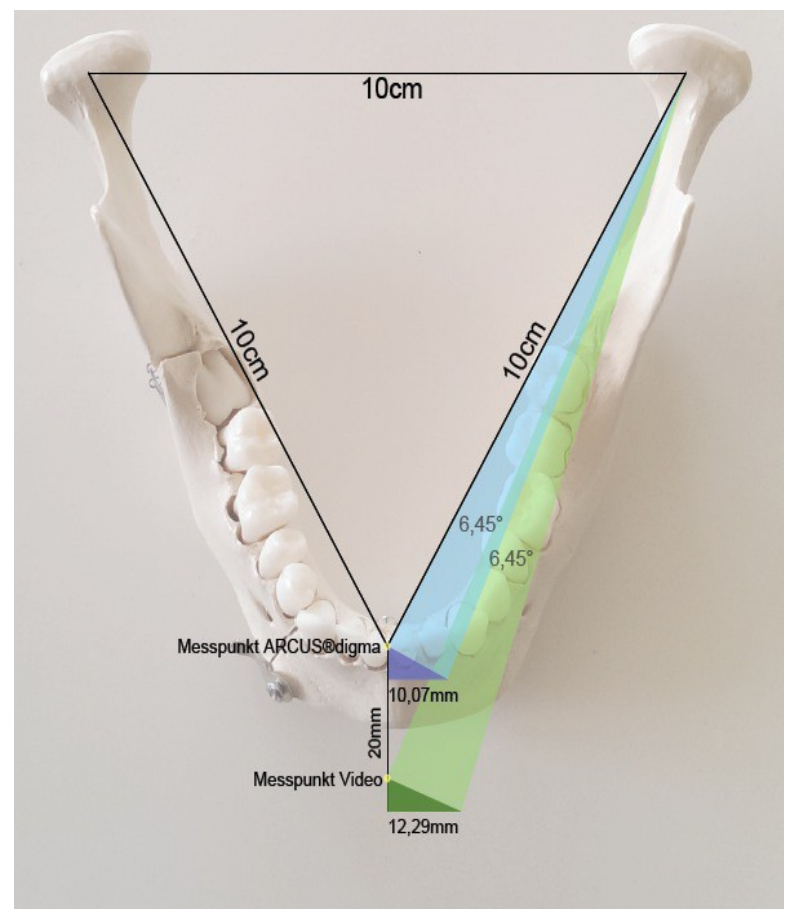

Abb. 23: Grafische Darstellung der Vergleichsberechnung bei einem Messpunktabstand von $2 \mathrm{~cm}$.

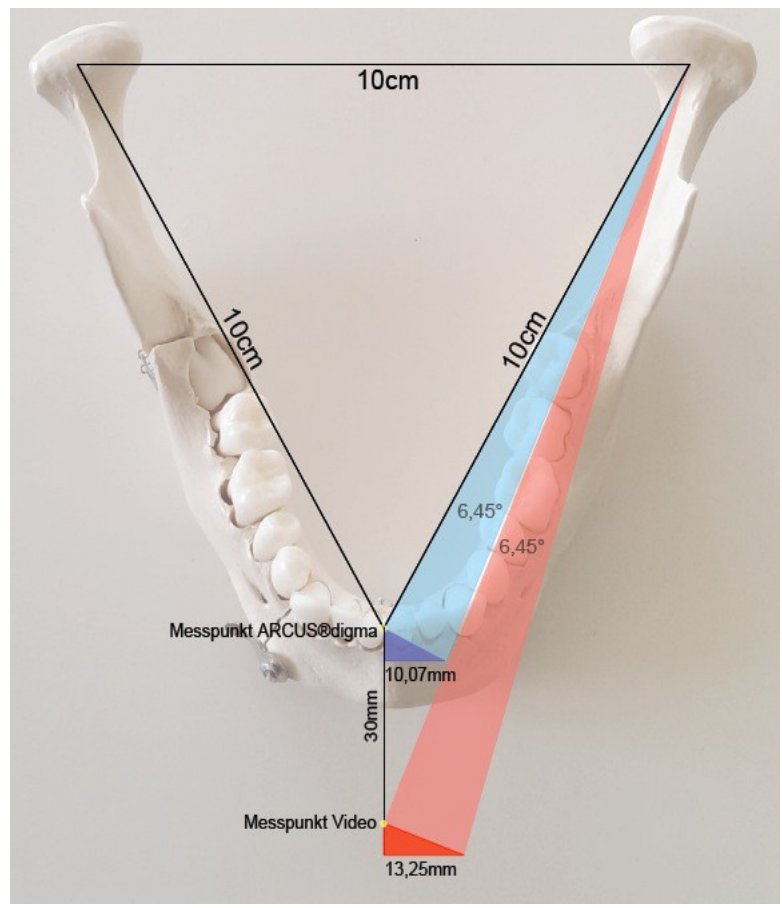

Abb. 24: Grafische Darstellung der Vergleichsberechnung bei einem Messpunktabstand von $3 \mathrm{~cm}$.

\subsection{Bewertungskriterien der Graphen}

Neben den Bewegungsmaxima-Werten ist auch die Form der einzelnen Bewegungsabschnitte wichtig für die Diagnose einer CMD. Die Maximalwerte können nur eine Bewertung im Bereich von Limitationen und Hypermobilitäten abgeben. Die Bewegungsbahnen zwischen den einzelnen Grenzbewegungen geben hingegen Aufschluss über mögliche Diskusversprünge und Kiefergelenkasymmetrien.

Aus diesem Grund wurden die einzelnen Bewegungsabschnitte bewertet und die Ergebnisse aus den ARCUS®digma-Graphen mit denen der Videoaufzeichnungen verglichen.

Für die Bewertung wurde eine Tabelle mit Parametern aufgestellt, die sowohl für die Kurven des ARCUS®digma sowie für die der Videoaufnahmen benutzt wurden (siehe Anhang 9.4 Bewertungskriterientabelle).

Ein Parameter war beispielsweise „Laterotrusion left“, der mit konkav, konvex oder gerade beschrieben werden konnte. Jeder Antwortmöglichkeit wurde eine Zahl zugeordnet (kon$k a v=1$, konvex $=2$ oder gerade = 3). Bei den Parametern „Right closing“ und „Left closing“ lauteten die Kurvenbeschreibungen Deflexion rechts (1), Deflexion links (2), Deviati- 
on rechts (3), Deviation links (4) und gerade (7). Da auch Bewegungskombinationen auftreten, wurden außerdem die Einstufungen Deflexion rechts mit Deviation (5) und Deflexion links mit Deviation (6) hinzugefügt.
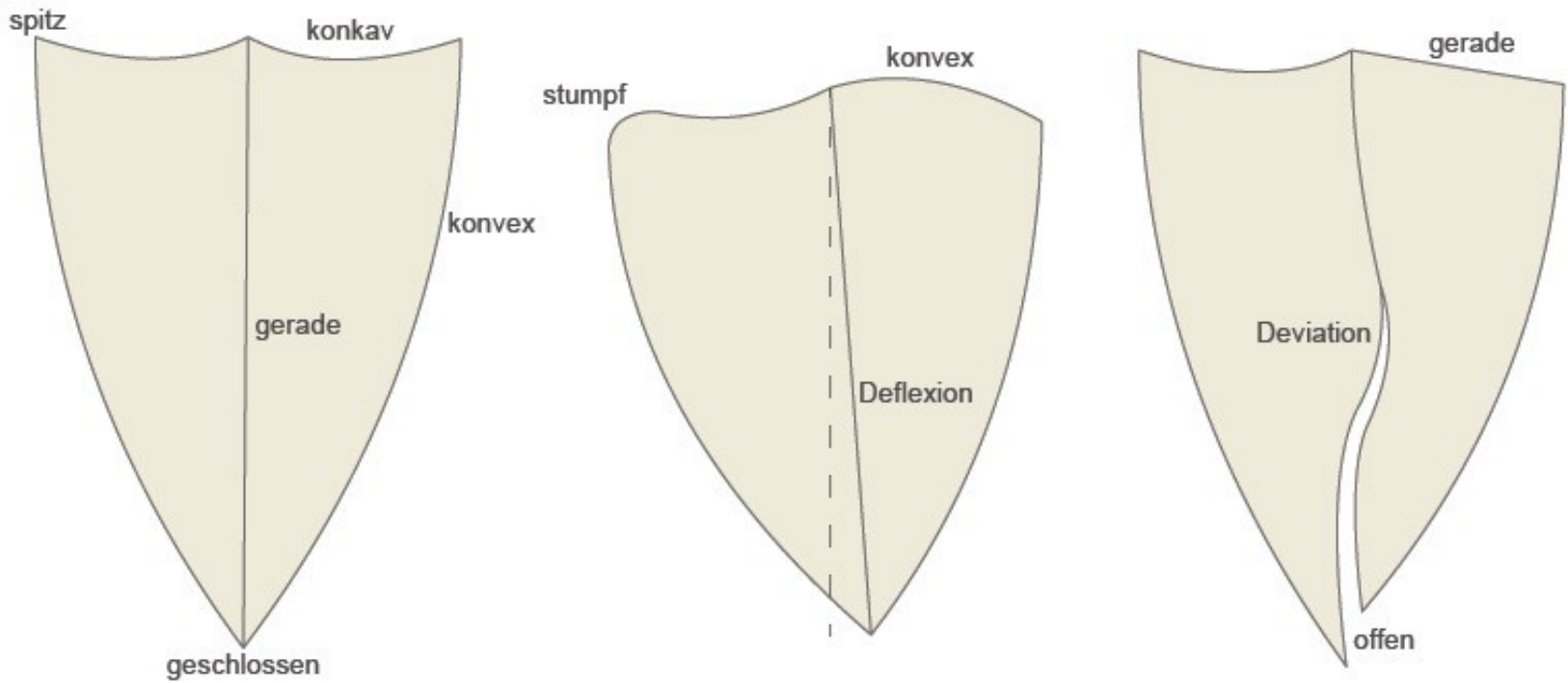

Abb. 25: Grafische Darstellung der möglichen Bewegungsabweichungen.

Die Tabelle mit den entsprechenden Zahlenwerten wurde in das Programm STATISTICA $®$ übertragen. Diese Software erkennt gleiche Zahlenwerte und kann somit die Übereinstimmungen berechnen.

Da die Kurvenbeurteilung keiner unabhängigen Methode zugrunde liegt und die Bewertung nur von einer Person durchgeführt wurde, sind die Ergebnisse eher explorativer Natur und nicht grundlegend wissenschaftlich aufzufassen. 


\subsection{Statistische Methodik}

\subsubsection{Deskriptive Auswertung}

Bewegungsmaxima-Werte: Um den Vergleich der Methoden grafisch darzustellen, werden die Daten des ARCUS®digma und der Videoanalyse innerhalb eines Koordinatensystems wiedergegeben.

Jede Bewegung wurde von den Probanden fünf Mal wiederholt und aus diesen fünf Messwerten die intraindividuellen Mediane gebildet. In den Grafiken sind diese intraindividuellen Mediane der beiden Messmethoden abgebildet. Die Whiskers markieren die Minimal- und Maximalwerte. Die verschiedenen Bewegungen (Laterotrusion links, Laterotrusion rechts, Mundöffnung-frontal, Mundöffnung-sagittal und Protrusion) sind dabei in separaten Plots aufgeführt.

Um einen besseren Überblick über die Ergebnisse zu erhalten, werden die Mittelwerte in einer zweiten Grafik durch eine Linie verbunden. Diese Verbindungslinie hat keinen inhaltlichen Bezug, sondern soll lediglich Übereinstimmungen und Abweichungen der Werte veranschaulichen.

Mittelwerte der Differenzen: Für die Darstellung der durchschnittlichen Abweichung der Methoden, wurden für jede Messung die Differenzen aus den Videografie- und den Gesichtsbogenwerten ermittelt (ARCUS®digmawerte - Videowerte). In den Grafiken werden diese Differenzwerte in Form von intraindividuellen Mittelwerten wiedergegeben.

Die horizontalen Geraden beschreiben zum einen die Nullhypothese und zum anderen den Mittelwert aller Differenzen innerhalb einer Grafik.

Vergleichsberechnung: Ergänzend wurde geprüft, ob die Werte der Laterotrusionsbewegung der Videografie mit denen einer theoretischen Maximaberechnung übereinstimmen. Die Grafiken zeigen die intraindividuellen Mediane der ARCUSßdima-Daten, der Videoanalysedaten sowie der Werte der Faktorenrechnung. Diese Darstellung wurde für die Bewegungen Laterotrusion links, Laterotrusion rechts und sagittale Mundöffnung angefertigt. 
Bewertungskriterien: Aus den Tabellenwerten der Kriterienliste wurde die prozentuale Übereinstimmung der Werte berechnet.

\subsubsection{Statistische Testverfahren}

Um die Hypothese zu überprüfen, ob die erwarteten Mittelwerte der beiden angewandten Verfahren sich nicht unterscheiden, wurde ein abhängiger T-Test durchgeführt.

Für alle statistischen Testverfahren wurde ein Signifikanzniveau von alpha $=0,05$ verwendet. Ein Wert von $\mathrm{p}<0,05$ gibt an, dass die Nullhypothese $(\mathrm{HO})$ nicht zutrifft und somit ein signifikanter Unterschied der betrachteten Methoden vorliegt (Rasch et al. 2010). 


\section{Ergebnisse}

\subsection{Bewegungsmaxima-Werte}

Die grafische Gegenüberstellung der Gesichtsbogen- und der Videografiemaxima zeigt für die Bewegungen Laterotrusion links und Laterotrusion rechts durchgängig höhere intraindividuelle Mediane für die Videoanalyse. Für die aus frontaler Sicht betrachtete Mundöffnung liegen 20 von 32 Probandenwerten des ARCUS®digma über den Videografiewerten. In den Grafiken für die Mundöffnung in der sagittalen Ebene und für die Protrusion liegen die Werte der Videoaufnahme mit 25 von 32 bzw. 26 von 32 Werten im Durchschnitt über den ARCUS®digma-Medianen.

Aus diesen Ergebnissen lässt sich ablesen, dass die Maxima-Werte der Videografie für die Bewegungen Laterotrusion links, Laterotrusion rechts, Mundöffnung-sagittal und Protrusion im Durchschnitt größer sind, als die Werte des elektronischen Gesichtsbogens. Die Werte für die aus der Frontalen betrachtete Mundöffnung sind hingegen im Durchschnitt kleiner, als die des ARCUS®digma.

Die Minimal- und Maximalwerte der Videografie zeigen im Vergleich zu den Gesichtsbogenwerten in den meisten Fällen ein deutlich vergrößertes Spektrum.

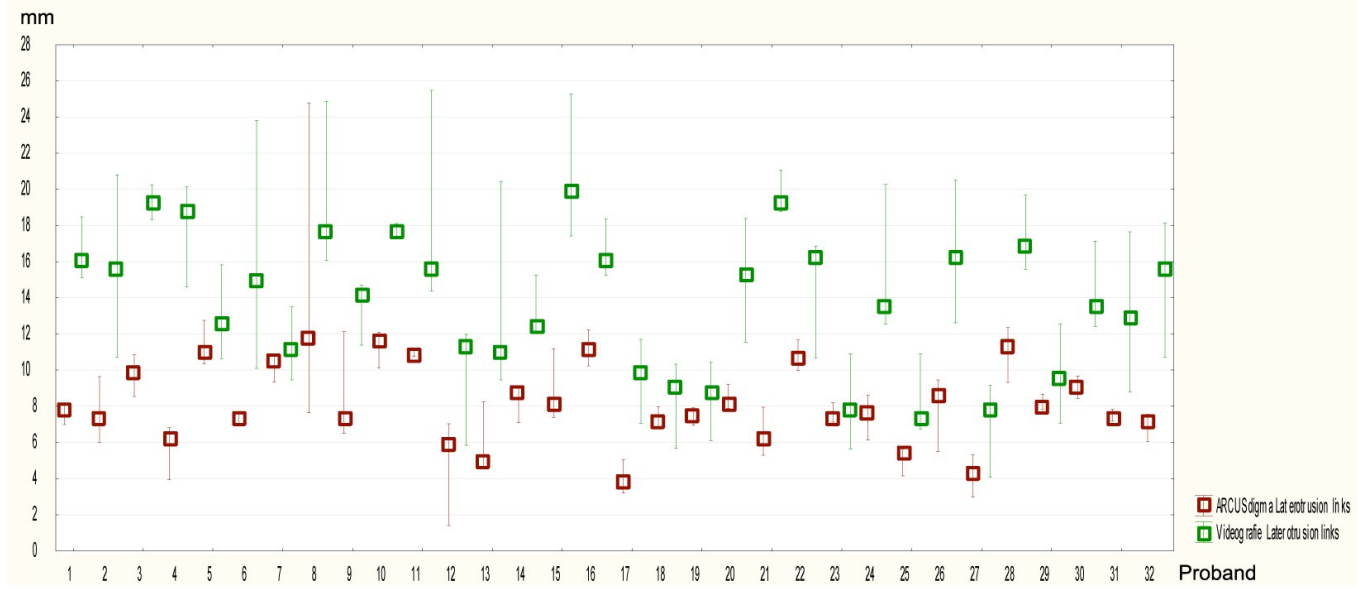

Abb. 26: Laterotrusion links: Darstellung der intraindividuellen Mediane für die Videoanalyse und den ARCUS®digma. Die Whiskers markieren die Minimal- und Maximalwerte. 


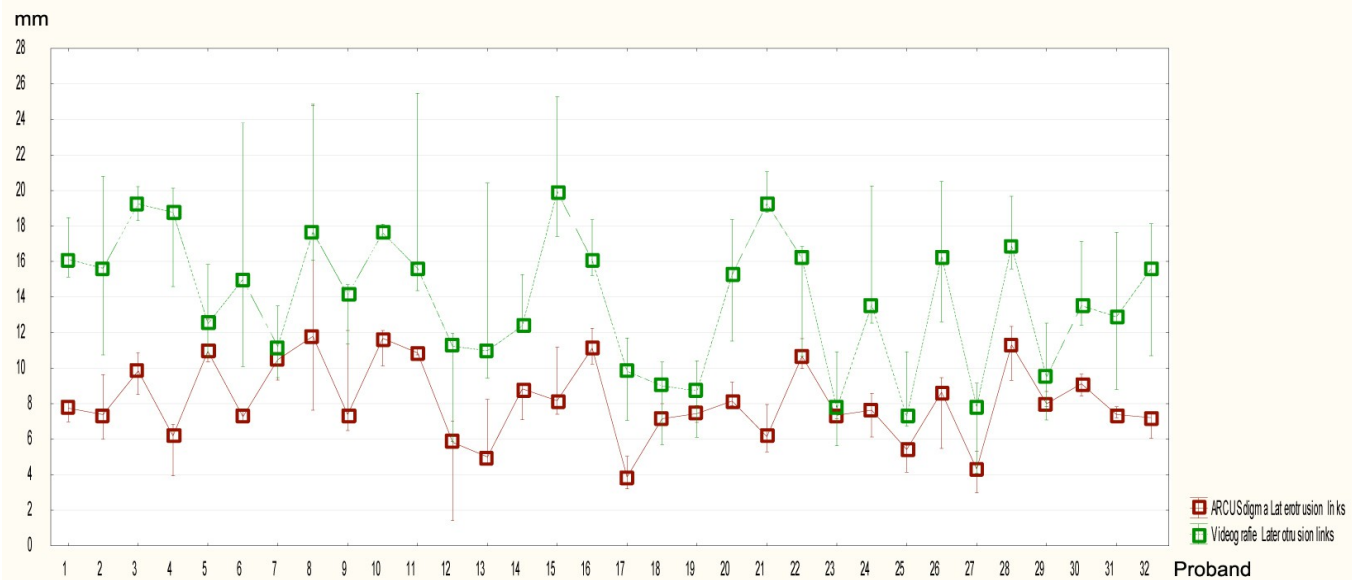

Abb. 27: Laterotrusion links: Darstellung der verbundenen intraindividuellen Mediane für die Videoanalyse und den ARCUS®digma. Die Whiskers markieren die Minimal- und Maximalwerte.

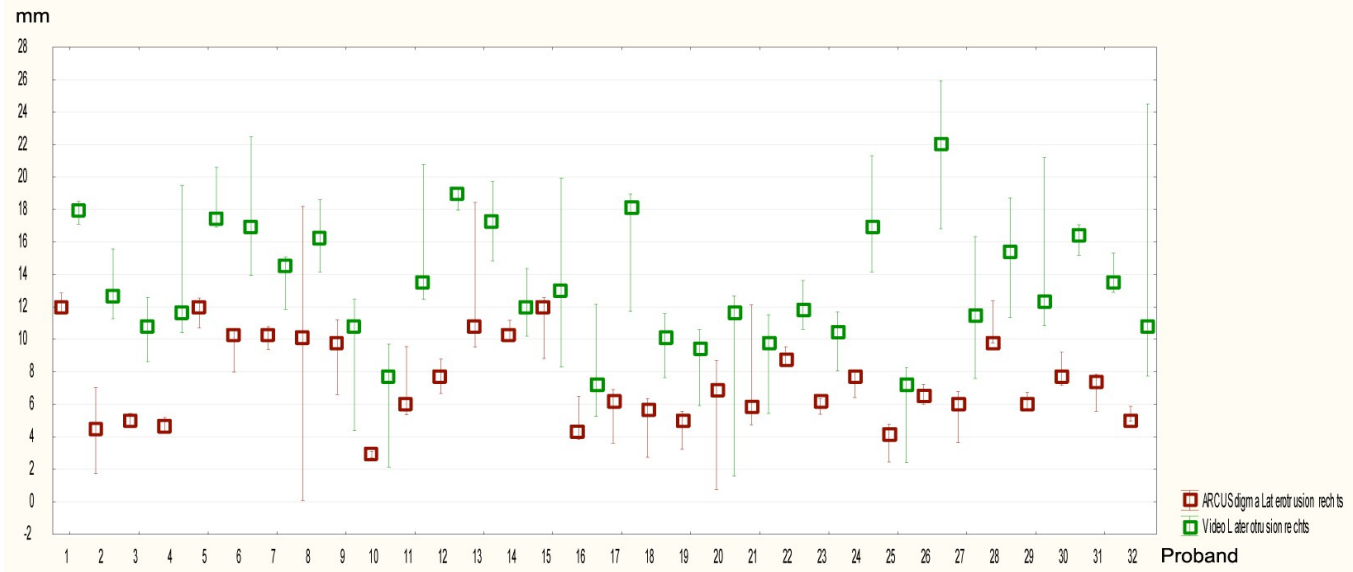

Abb. 28: Laterotrusion rechts: Darstellung der intraindividuellen Mediane für die Videoanalyse und den ARCUS®digma. Die Whiskers markieren die Minimal- und Maximalwerte.

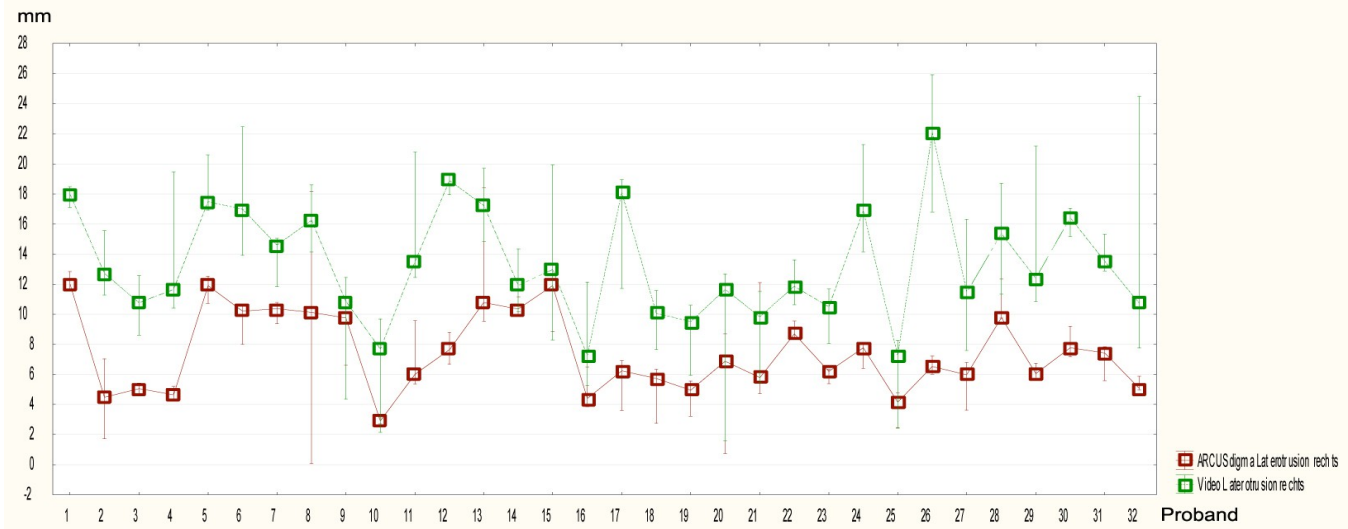

Abb. 29: Laterotrusion rechts: Darstellung der verbundenen intraindividuellen Mediane für die Videoanalyse und den ARCUS $®$ digma. Die Whiskers markieren die Minimal- und Maximalwerte. 


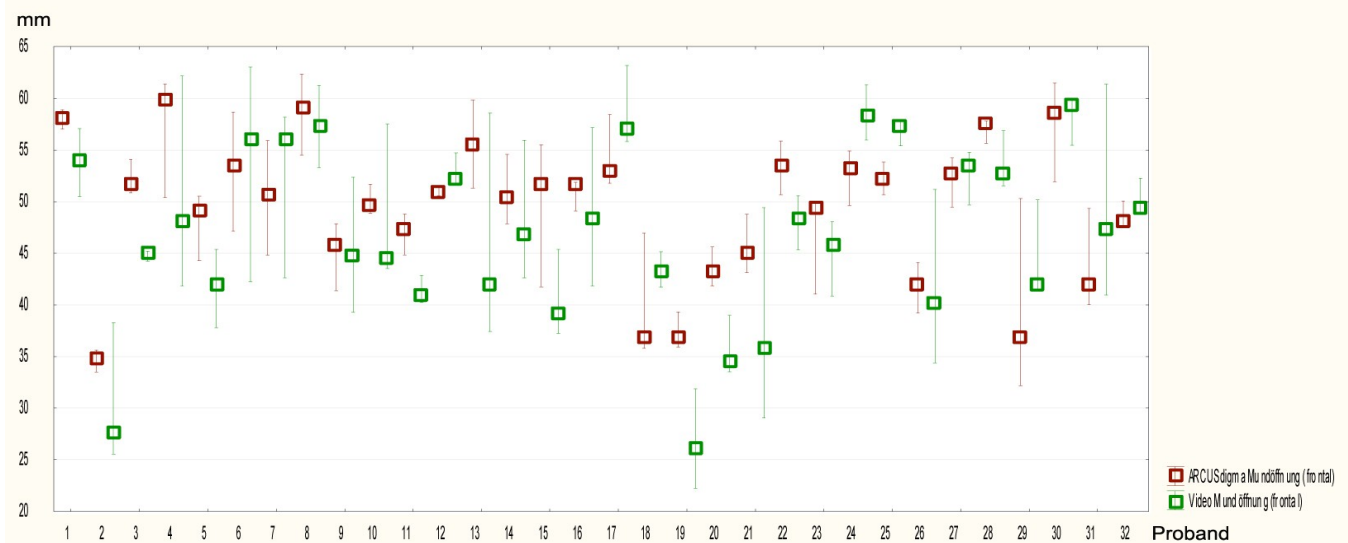

Abb. 30: Mundöffnung frontal: Darstellung der intraindividuellen Mediane für die Videoanalyse und den ARCUS ${ }^{\circ}$ digma. Die Whiskers markieren die Minimal- und Maximalwerte.

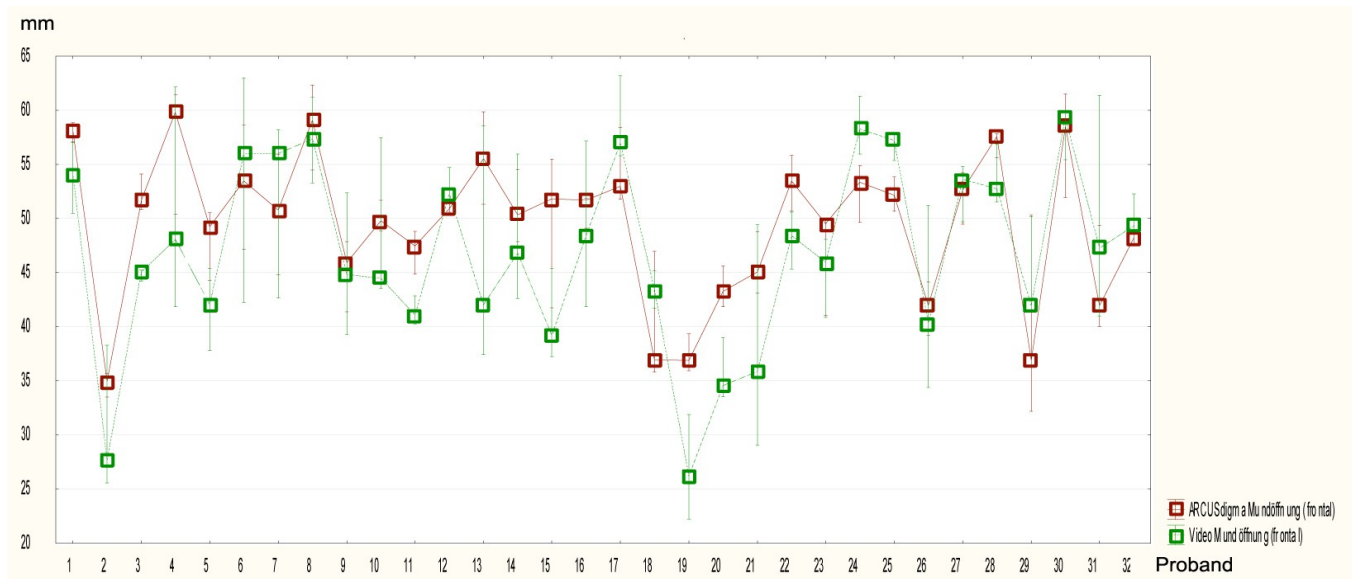

Abb. 31: Mundöffnung frontal: Darstellung der verbundenen intraindividuellen Mediane für die Videoanalyse und den ARCUS $®$ digma. Die Whiskers markieren die Minimal- und Maximalwerte.

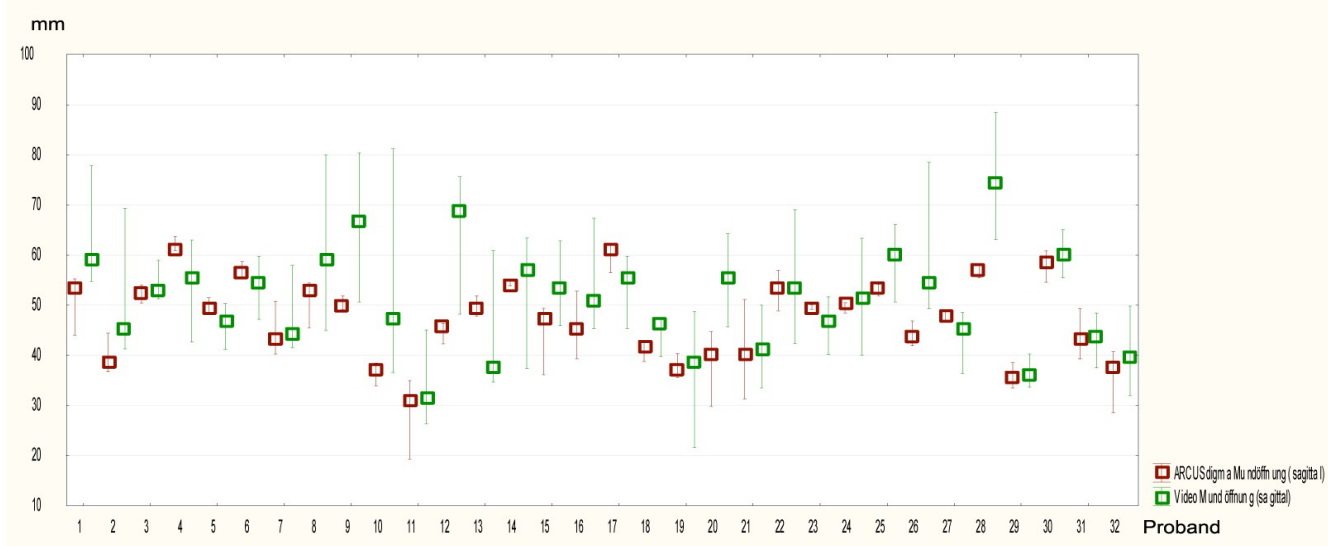

Abb. 32: Mundöffnung sagittal: Darstellung der intraindividuellen Mediane für die Videoanalyse und den ARCUS $®$ digma. Die Whiskers markieren die Minimal- und Maximalwerte. 


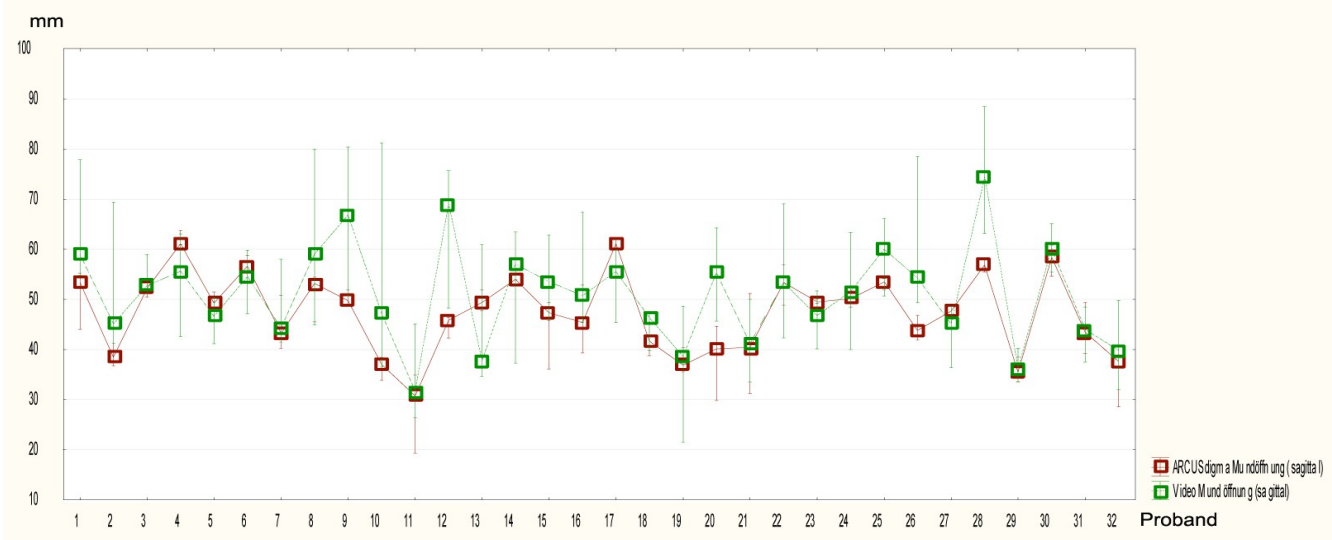

Abb. 33: Mundöffnung sagittal: Darstellung der verbundenen intraindividuellen Mediane für die Videoanalyse und den ARCUS $®$ digma. Die Whiskers markieren die Minimal- und Maximalwerte.

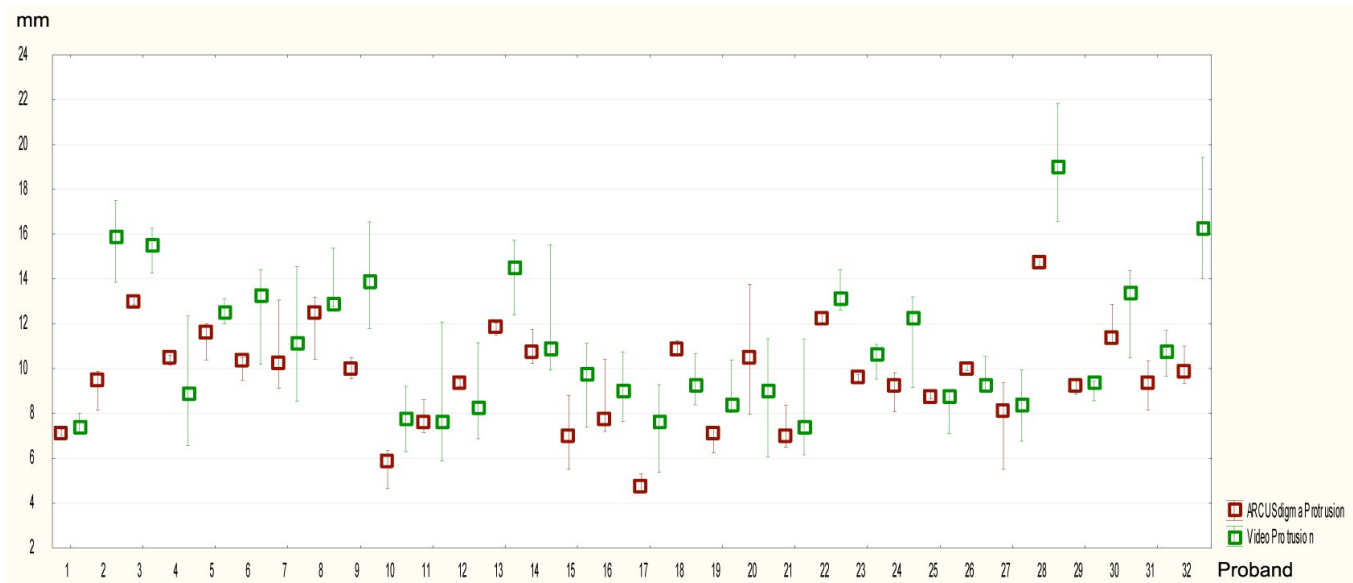

Abb. 34: Protrusion: Darstellung der intraindividuellen Mediane für die Videoanalyse und den ARCUS $囚$ digma. Die Whiskers markieren die Minimal- und Maximalwerte.

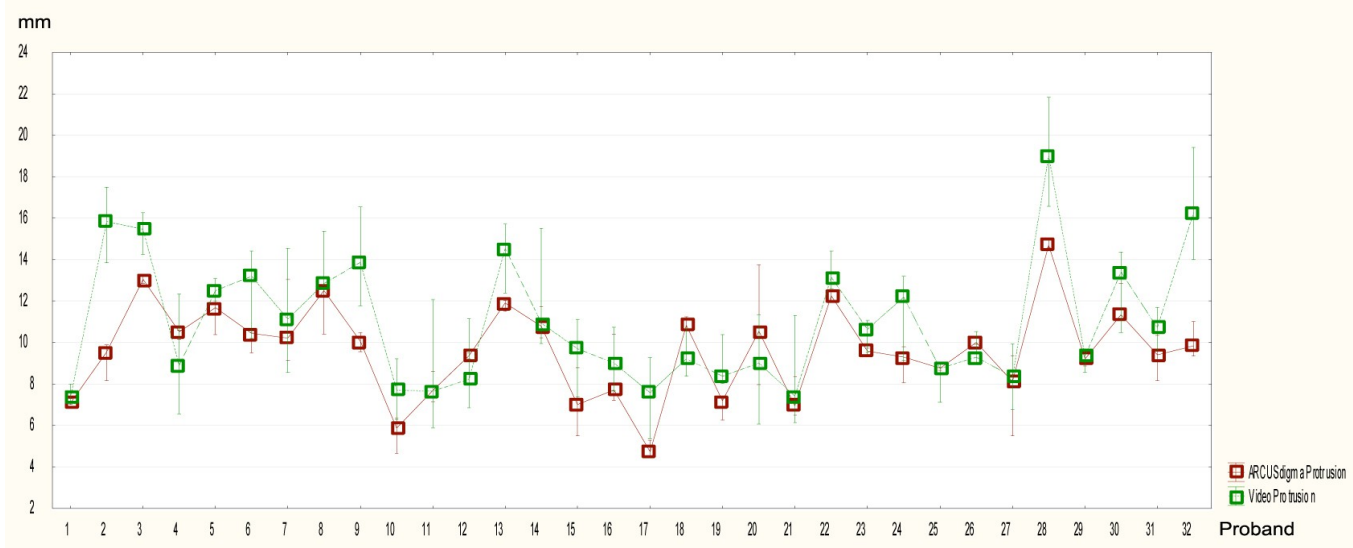

Abb. 35: Protrusion: Darstellung der verbundenen intraindividuellen Mediane für die Videoanalyse und den ARCUS $®$ digma. Die Whiskers markieren die Minimal- und Maximalwerte. 


\begin{tabular}{|l|l|l|l|l|l|}
\hline Bewegung & $\begin{array}{l}\text { Laterotrusion } \\
\text { links }\end{array}$ & $\begin{array}{l}\text { Laterotrusion } \\
\text { rechts }\end{array}$ & $\begin{array}{l}\text { frontale Mund- } \\
\text { öffnung }\end{array}$ & $\begin{array}{l}\text { sagittale Mund- } \\
\text { öfnung }\end{array}$ & Protrusion \\
\hline ARCUS®digma & $8,21 \mathrm{~mm}$ & $7,4 \mathrm{~mm}$ & $49,26 \mathrm{~mm}$ & $47,23 \mathrm{~mm}$ & $9,6 \mathrm{~mm}$ \\
\hline Videoanalyse & $14,04 \mathrm{~mm}$ & $13,33 \mathrm{~mm}$ & $47,23 \mathrm{~mm}$ & $51,78 \mathrm{~mm}$ & $11 \mathrm{~mm}$ \\
\hline
\end{tabular}

Tab. 1: Mittelwerte der Grenzbewegungen.

\subsection{Mittelwerte der Differenzen}

Die Mittelwerte der Differenzen betragen für die Bewegungen Laterotrusion links $-5,83 \mathrm{~mm}$, Laterotrusion rechts $-5,95 \mathrm{~mm}$, Mundöffnung aus der Frontalen 2,03 mm, Mundöffnung aus der Sagittalen $-4,5 \mathrm{~mm}$ und für die Protrusion -1,38 mm.

Bis auf die Werte der frontalen Mundöffnung liegen alle gemittelten Differenzen im negativen Bereich. Dieses Ergebnis zeigt, dass die Bewegungsmaxima-Werte der Videoanalyse für diese Bewegungen im Durchschnitt größer sind. Die Werte für die frontal gemessene Mundöffnung sind hingegen durchschnittlich kleiner, als die der Gesichtsbogenmaxima.

Der T-Test zeigt für alle Bewegungen, dass die Unterschiede der Mittelwerte signifikant sind $(p<0,05)$.

\begin{tabular}{|l|r|c|}
\hline & Mittelwerte & \multicolumn{1}{c|}{$\mathrm{p}$} \\
\hline Laterotrusion links & $-5,82881$ & 0,000000 \\
\hline Laterotrusion rechts & $-5,94794$ & 0,000000 \\
\hline Mundöffnung frontal & 2,02686 & 0,026999 \\
\hline Mundöffnung sagittal & $-4,49607$ & 0,002542 \\
\hline Protrusion & $-1,38074$ & 0,000338 \\
\hline
\end{tabular}

Tab. 2: Mittelwerte der Differenzen und $p$ (T-Test). 


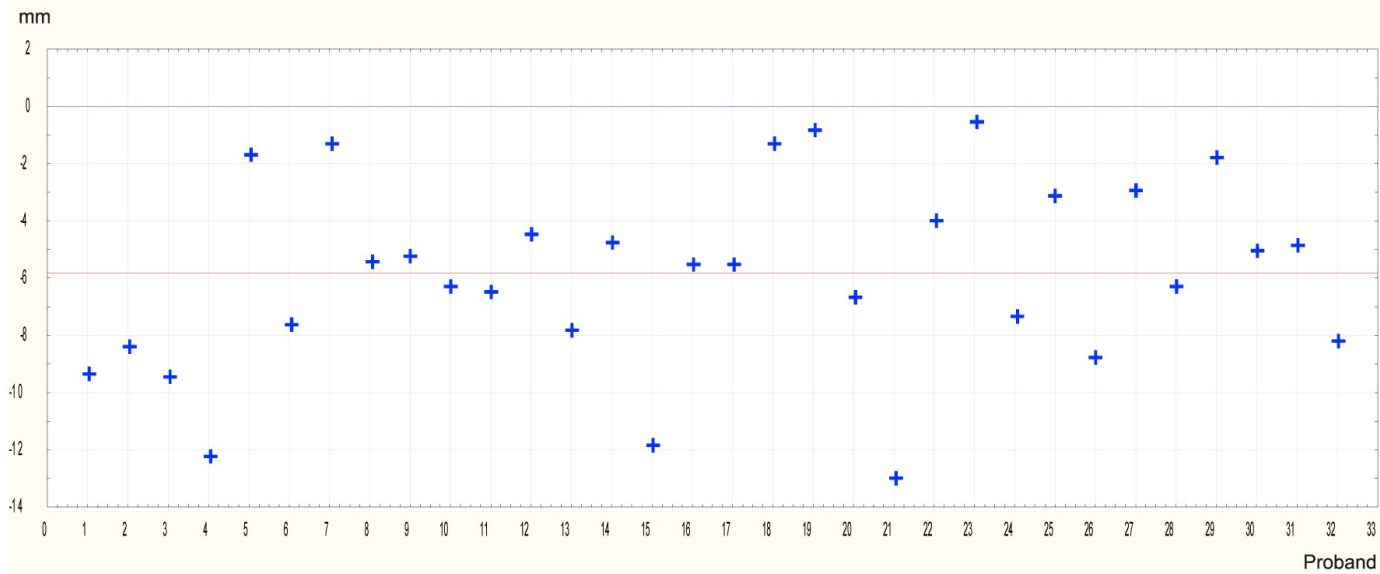

Abb. 36: Laterotrusion links: Intraindividuelle Differenzmittelwerte der Videografie- und Gesichtsbogenwerte. Die horizontalen Geraden beschreiben zum einen die Nullhypothese (blau) und zum anderen den Mittelwert aller Differenzen innerhalb einer Grafik (rot).

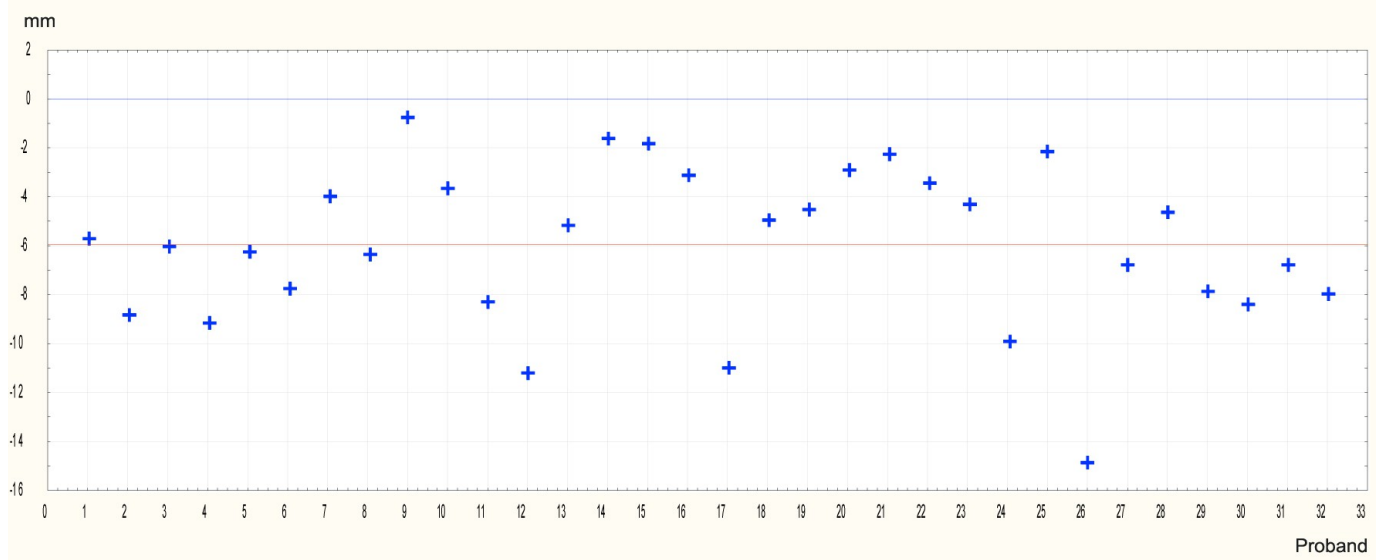

Abb. 37: Laterotrusion rechts: Intraindividuelle Differenzmittelwerte der Videografieund Gesichtsbogenwerte. Die horizontalen Geraden beschreiben zum einen die Nullhypothese (blau) und zum anderen den Mittelwert aller Differenzen innerhalb einer Grafik (rot).

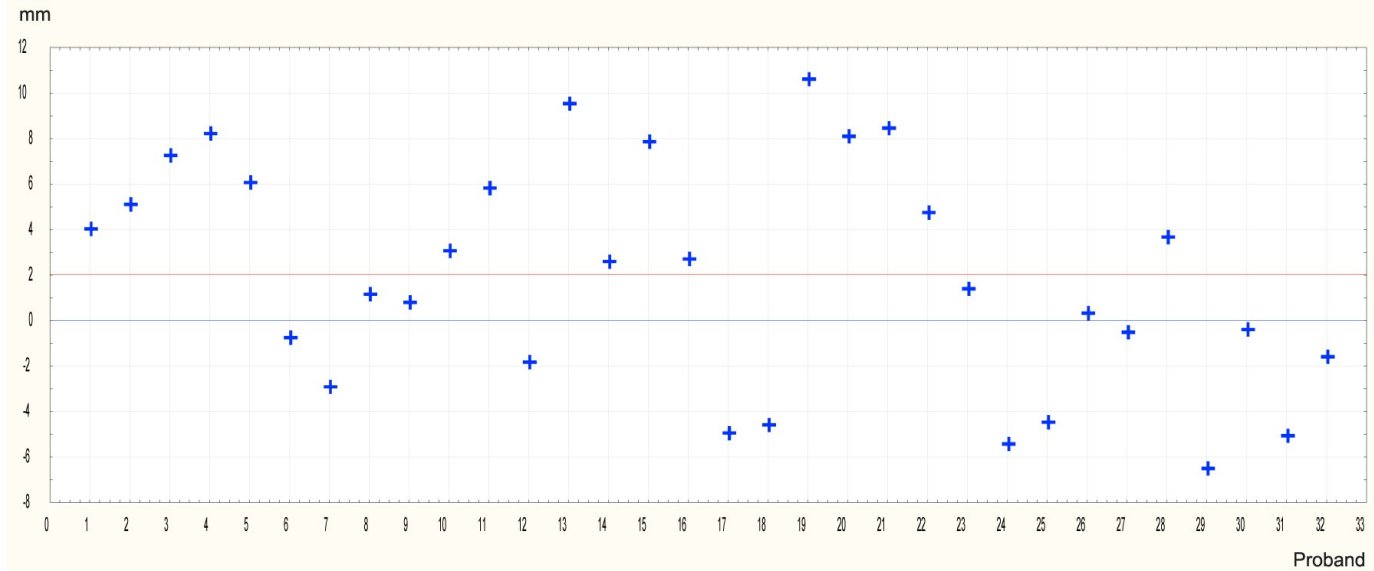

Abb. 38: Mundöffnung-frontal: Intraindividuelle Differenzmittelwerte der Videografieund Gesichtsbogenwerte. Die horizontalen Geraden beschreiben zum einen die Nullhypothese (blau) und zum anderen den Mittelwert aller Differenzen innerhalb einer Grafik (rot). 


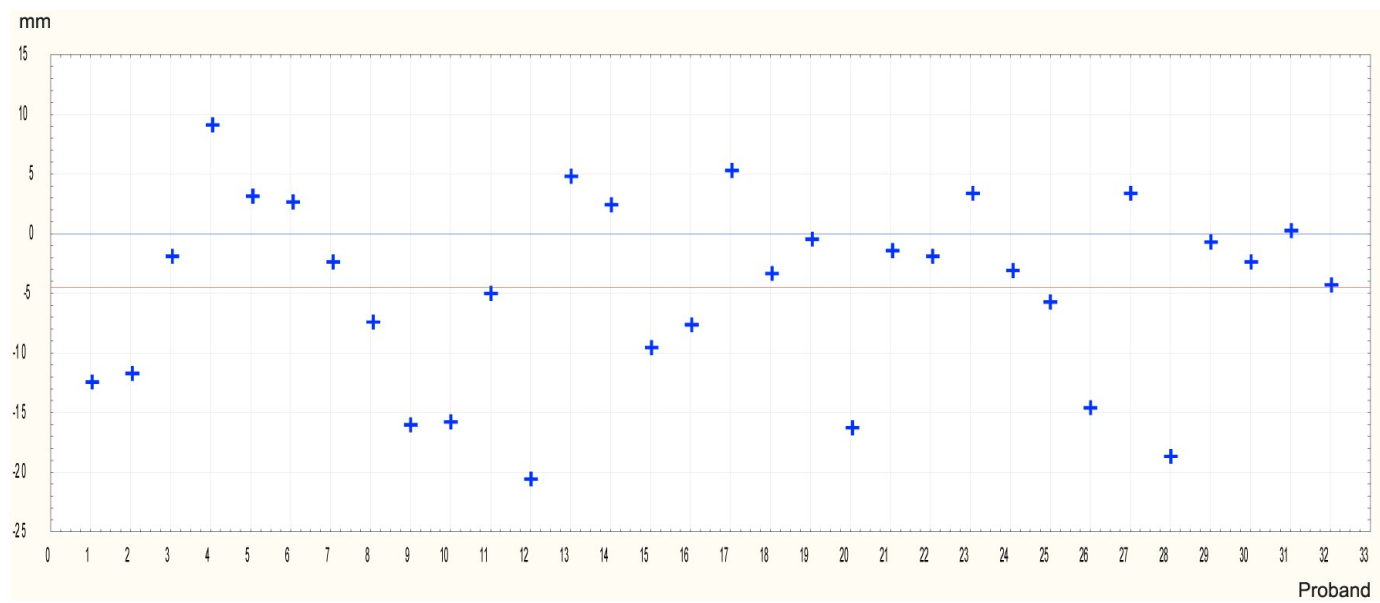

Abb. 39: Mundöffnung-sagittal: Intraindividuelle Differenzmittelwerte der Videografieund Gesichtsbogenwerte. Die horizontalen Geraden beschreiben zum einen die Nullhypothese (blau) und zum anderen den Mittelwert aller Differenzen innerhalb einer Grafik (rot).

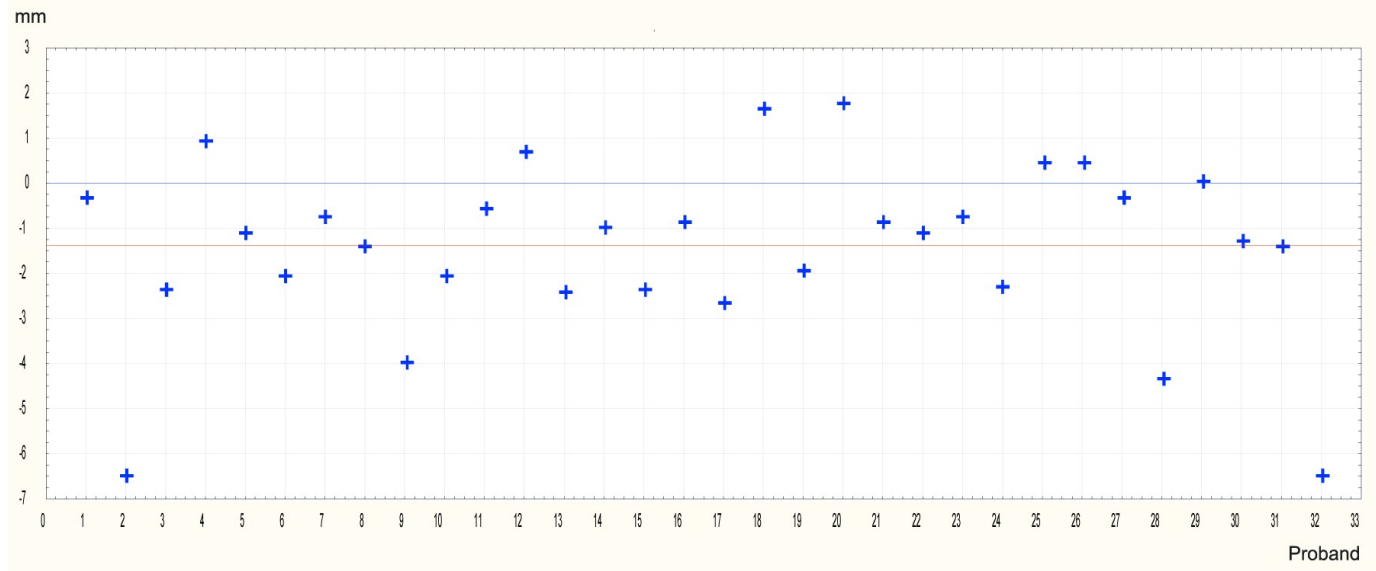

Abb. 40: Protrusion: Intraindividuelle Differenzmittelwerte der Videografie- und Gesichtsbogenwerte. Die horizontalen Geraden beschreiben zum einen die Nullhypothese (blau) und zum anderen den Mittelwert aller Differenzen innerhalb einer Grafik (rot).

\subsection{Vergleichsberechnung}

Die Grafiken zeigen, dass sich die Kurven der theoretischen Werte den tatsächlichen Videografiemaxima annähern. Viele Messwerte zeigen aber nicht die gewünschte Übereinstimmung. Bei den Messdaten ist zu erkennen, dass die theoretisch berechneten Mediane zum Teil unter den tatsächlichen Werten der Videografie und zum Teil über diesen liegen. 


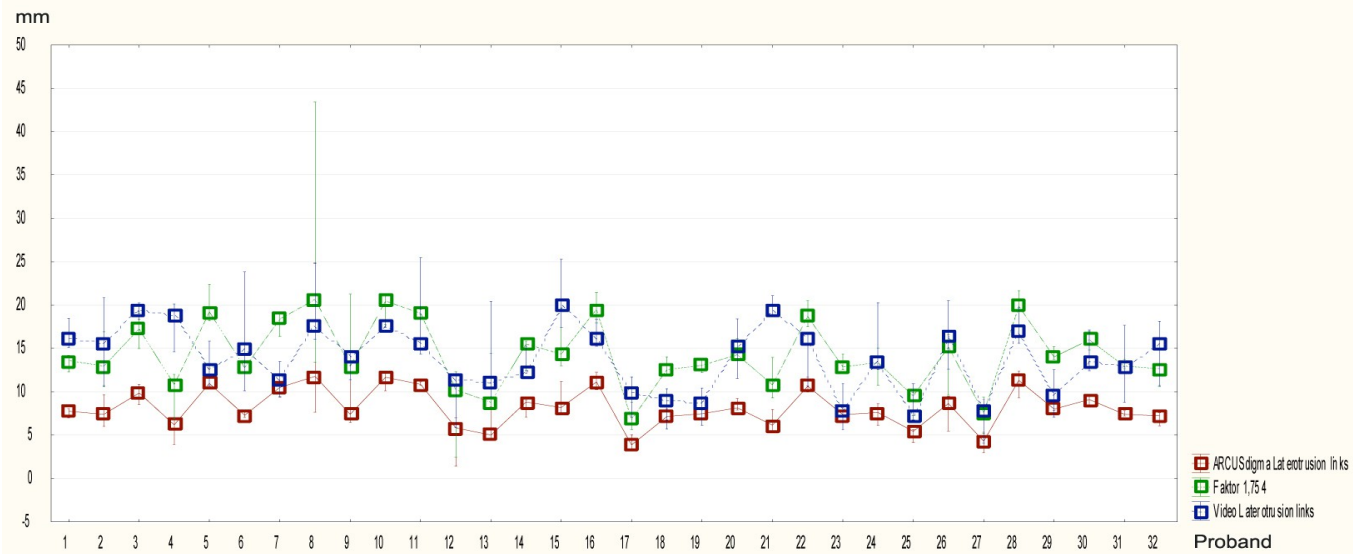

Abb. 41: Laterotrusion links: Die Grafiken zeigen die intraindividuellen Mediane der ARCUSßdimadaten (rot), der Videoanalysedaten (blau) sowie der Werte der Faktorenrechnung (grün).

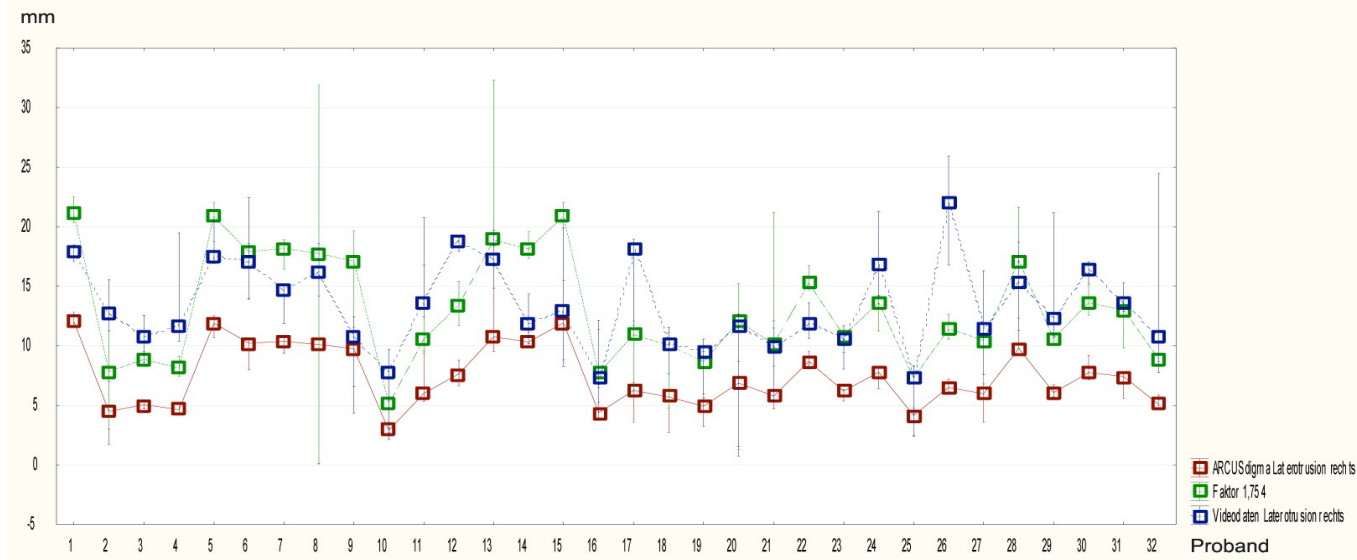

Abb. 42: Laterotrusion rechts: Die Grafiken zeigen die intraindividuellen Mediane der ARCUSßdimadaten (rot), der Videoanalysedaten (blau) sowie der Werte der Faktorenrechnung (grün).

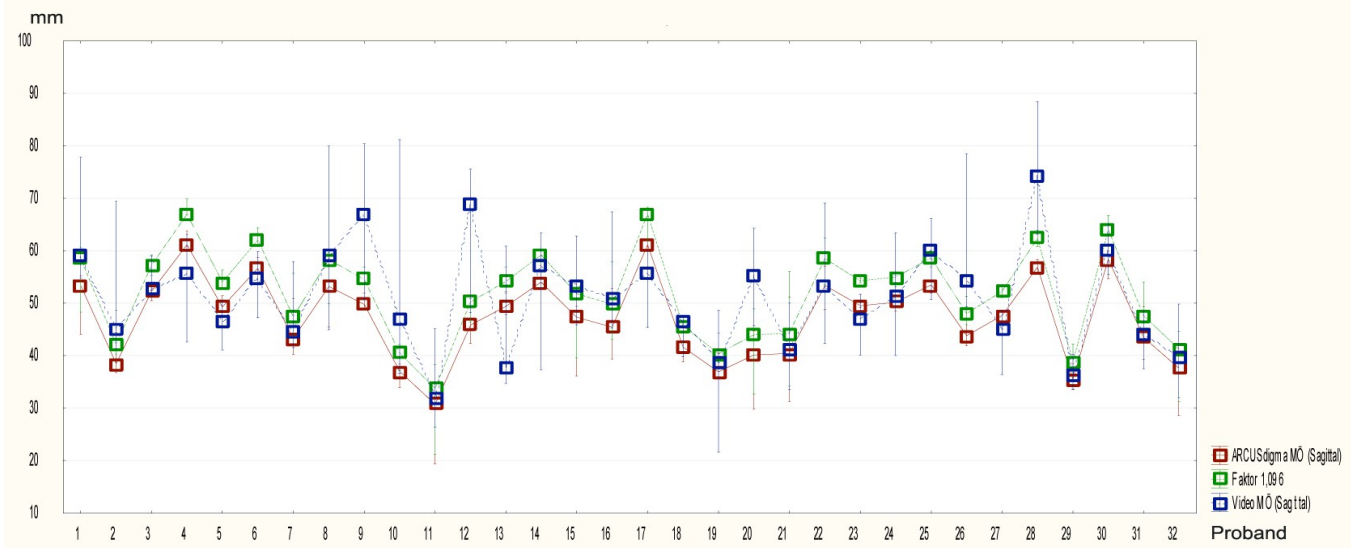

Abb. 43: Mundöffnung sagittal: Die Grafiken zeigen die intraindividuellen Mediane der ARCUS®dimadaten (rot), der Videoanalysedaten (blau) sowie der Werte der Faktorenrechnung (grün). 


\subsection{Bewertungskriterien}

Die Graphen der beiden untersuchten Messmethoden zeigen in ihrer Form viele Gemeinsamkeiten. Die prozentuale Übereinstimmung der einzelnen Bewegungsabschnitte liegt zwischen $51 \%$ (Closing endpoints - Posselt sagittal) und $96 \%$ (Closing - Posselt sagittal). Im Durchschnitt liegt die Übereinstimmung bei 80 \%.

\begin{tabular}{|l|l|l|l|l|l|}
\hline Laterotrusion left & Left endpoint & Left opening & $\begin{array}{l}\text { Left opening } \\
\text { endpoint }\end{array}$ & Left closing & $\begin{array}{l}\text { Laterotrusion } \\
\text { right }\end{array}$ \\
\hline $88,4 \%$ & $86,4 \%$ & $83,2 \%$ & $93,5 \%$ & $52,9 \%$ & $81,9 \%$ \\
\hline
\end{tabular}

\begin{tabular}{|l|l|l|l|l|l|}
\hline Right endpoint & Right opening & $\begin{array}{l}\text { Right opening } \\
\text { endpoint }\end{array}$ & Right closing & $\begin{array}{l}\text { Opening } \\
\text { endpoint }\end{array}$ & Closing endpoint \\
\hline $88,9 \%$ & $81,2 \%$ & $93,5 \%$ & $52,8 \%$ & $56,1 \%$ & $86,4 \%$ \\
\hline
\end{tabular}

Tab. 3: Die prozentuale Übereinstimmung für die frontalen Bewegungsabschnitte.

\begin{tabular}{|l|l|l|l|l|l|}
\hline Protrusion & $\begin{array}{l}\text { Protrusion } \\
\text { endpoint }\end{array}$ & Opening & $\begin{array}{l}\text { Opening } \\
\text { endpoint }\end{array}$ & Closing & Closing endpoint \\
\hline $81,1 \%$ & $93,5 \%$ & $85,5 \%$ & $92,9 \%$ & $96 \%$ & $51,1 \%$ \\
\hline
\end{tabular}

Tab. 4: Die prozentuale Übereinstimmung für die sagittalen Bewegungsabschnitte. 


\section{Diskussion}

\subsection{Zusammenfassung der Hauptergebnisse}

Bewegungsmaxima: Die Vergleiche der Bewegungsmaxima beider Methoden ergaben, dass die Werte der Laterotrusion links, Laterotrusion rechts, der Mundöffnung aus der Sagittalen und der Protrusion für die Videografie im Durchschnitt über den Mittelwerten der Gesichtsbogenmessungen liegen. Die Videoanalyse-Messwerte für die aus der Frontalen aufgezeichnete Mundöffnung liegen hingegen durchschnittlich unter den ARCUS®digmaWerten.

Mittelwerte der Differenzen: Der im Zuge der Berechnung der Mittelwerte der Differenzen durchgeführte T-Test zeigt, dass die Unterschiede der Mittelwerte der beiden verglichenen Methoden signifikant sind.

Vergleichsberechnung: Die Durchführung der Vergleichsberechnung hat ergeben, dass sich die Messkurven der beiden untersuchten Systeme angleichen. Eine Kongruenz der Graphen ist aber nicht gegeben.

Bewertungskriterien: Anhand von festgelegten Bewertungskriterien wurde die Form der Graphen beider Messmethoden untersucht. Die Untersuchung hat ergeben, dass sich die Graphen in ihrer Form zu 80 \% entsprechen.

Da noch keine Studie durchgeführt wurde, in der eine elektronische Bewegungsanalyse mit einer Videoanalyse verglichen wurde, können keine direkten Vergleiche der Ergebnisse dieser Studie mit anderen Studienergebnissen gezogen werden. 


\subsection{Diskussion der methodischen Aspekte}

Im folgenden Diskussionsteil werden zunächst die Methoden der Videoanalyse und des ARCUS $R$ digma kritisch betrachtet und abschließend beide Systeme verglichen und ihre Differenzen diskutiert.

\subsubsection{Videografie}

\subsubsection{Aufnahme der Bewegungen}

Kopfbewegungen: Während der Aufnahmen haben einige Probanden ihren Kopf zum Teil stark bewegt. Dies sollte durch zusätzliche Messpunkte auf der Stirn/Nasenspitze rechnerisch kompensiert werden. Es ist aber davon auszugehen, dass eine adäquate Fehlerbeseitigung auch durch die Subtraktion der Kopfbewegungen nicht immer möglich ist, da Abweichungen ausschließlich in der Frontalebene (für die frontalen Aufnahmen) beziehungsweise in der Sagittalebene (für die sagittalen Aufnahmen) ausgeglichen werden können. Bei den aus frontaler Sicht aufgenommenen Bewegungen können alle sagittalen Kopfbewegungen nicht berücksichtigt werden. Gleiches gilt entsprechend für die aus sagittaler Sicht aufgenommenen Bewegungen.

Nach Durchführung der Untersuchungen hat sich eine wichtige Änderung im Versuchsaufbau herausgestellt: Um den Kopf der Probanden still zu halten, sollte dieser in einer Position fixiert werden.

In der vorliegenden Untersuchung sollte auf jede invasive Maßnahme verzichtet werden. Aus diesem Grund wurde den Probanden lediglich gesagt, dass der Kopf während der Messungen nicht bewegt werden darf. Die Ergebnisse dieser Arbeit zeigen, dass vielen Personen das Stillhalten des Kopfes während der Durchführung einer bestimmten Bewegung des Unterkiefers nicht möglich ist. Dieses Untersuchungsergebnis unterstützt die Ergebnisse anderer Studien. Schon Thouren hat in seinen Analysen von 1914 die nötige Fixation des Kopfes angesprochen (Thouren 1914). Auch Hildebrand fixierte in seiner Studie von 1931 die Köpfe der Probanden (Hildebrand 1931). Beyron befestigte 1964 die Köpfe einiger Probanden, in dem innen ein Band um die Stirn gebunden wurde (Beyron 
1964). Bei anderen Studien erfolgte die Fixation durch ein Schaumstoffkissen (Furtado et al. 2013) oder eine Gipsschale (Goodson und Johansen 1975). In den meisten Studien wurden zusätzliche Referenzmarker angebracht, die es ermöglichen, die Kopfbewegung in einer Ebene zu kompensieren. Diese wurden auf der Stirn (Karlsson 1977; Pinheiro et al. 2011), der Nase (Atkinson und Shepherd 1955) oder an einem Kopfbogen angebracht (Mesqui und Palla 1985).

Aus den Untersuchungsergebnissen ist zu schließen, dass die Kombination aus fixiertem Kopf und zusätzlichen Referenzmarkern die größtmögliche Genauigkeit der Messungen zulässt. Deshalb soll hier empfohlen werden, diesen Versuchsaufbau für folgende Untersuchungen zu berücksichtigen.

Verdeckung des Kinnpunktes: Um die Bewegungsgraphen auf Übereinstimmung prüfen zu können, mussten sowohl die Messungen des ARCUS®digma als auch die Videoaufnahmen der Bewegungen zeitgleich durchgeführt werden. Durch die Anbringung des Gesichtsbogens wurde die Videoauswertung stark beeinflusst, da das Kinn bei der Mundöffnung zum Teil vollständig durch den Unterkieferbogen verdeckt wird. Darüber hinaus schiebt sich das mittig am Unterkiefergesichtsbogen angebrachte Kabel bei den Seitwärtsbewegungen einiger Probanden über den Kinnmarker der Videoanalyse. In der Auswertung der Videos führten diese Überlagerungen häufig zu Problemen und Verfälschungen, da das Programm Rastreobackup den Marker nicht mehr registrieren und somit auch nicht mehr verfolgen konnte.

Um die Verdeckung zu minimieren, wurde schon während der Durchführung der Untersuchungen bei den meisten Probanden mit Hilfe eines Pappstreifens ein zweiter Kinnmarker angebracht. Dieser befand sich etwas weiter kaudal als der erste. Kam es dennoch zur Verdeckung eines Messpunktes, wurde die Position des Markers für diesen Bewegungsabschnitt manuell festgelegt. Für diese Kurvenabschnitte handelt es sich also nur noch um wahrscheinliche Bewegungsabläufe.

Für folgende Untersuchungen ohne die parallele Anwendung eines Gesichtsbogens stellt sich dieses Problem nicht, da keine verdeckenden Geräte am Kopf des Probanden angebracht werden. 


\subsubsection{Analyse der Aufnahmen}

Videoformat: Zunächst sollten die Videosequenzen mit dem Programm Kinovea analysiert werden. Da aber die Frequenz der errechneten Einzelvektoren deutlich geringer ist (der Graph deutlich weniger Informationen enthält), als die des Programms Rastreobackup, wurde letzteres für die Analysen verwendet. Da das Programm Rastreobackup aber nicht in der Lage ist, das Sony-eigene Format .MTS zu lesen (Abb. 44), mussten alle Videos in das Format.AVI konvertiert werden. Durch diese Veränderung haben die Aufnahmen stark an Qualität verloren (Abb. 45).

Dies hatte Auswirkungen auf das Marker-Trekking während der Videoanalyse im Programm Rastreobackup, da die Marker bei schlechter Bildqualität mit einer geringeren Anzahl an Pixeln dargestellt werden und somit an Randschärfe verlieren. Dadurch ist auch der Kreismittelpunkt weniger klar definiert. Darüber hinaus war die Skalierung schlechter zu erkennen, was zu Ungenauigkeiten in der Markierung geführt haben kann. Da die Millimeterskala im Video das Verbindungsglied in der Umrechnung von Pixeln in Millimeter ist, kann eine Verschlechterung der Bildqualität große Auswirkungen auf die endgültigen Ergebnisse haben.

Auf Grundlage der Untersuchungen soll vorgeschlagen werden, dass für weiterführende Studien die Aufnahmen in einem anderen Format gespeichert werden sollten, um Qualitätseinbußen zu verhindern.

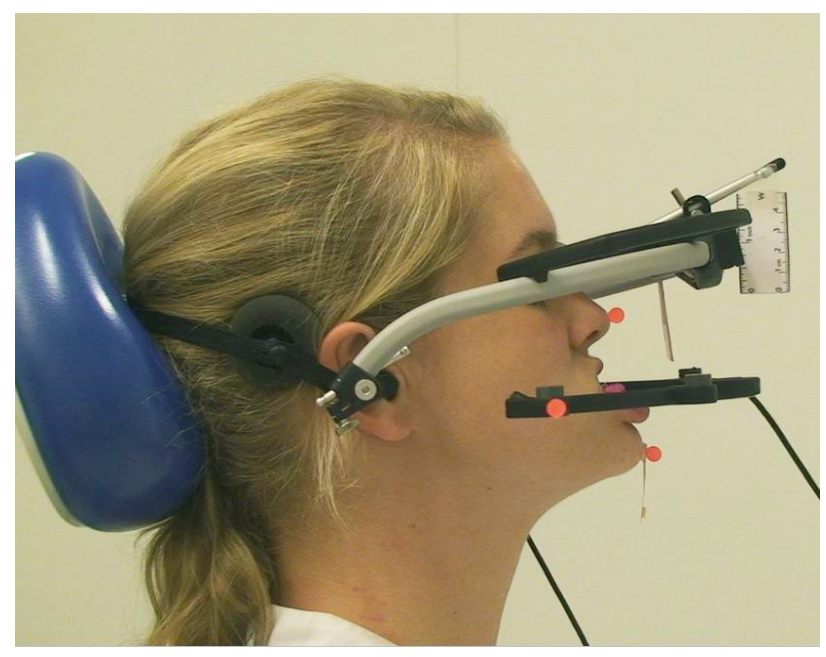

Abb. 44: Videoaufnahme im .MTS-Format.

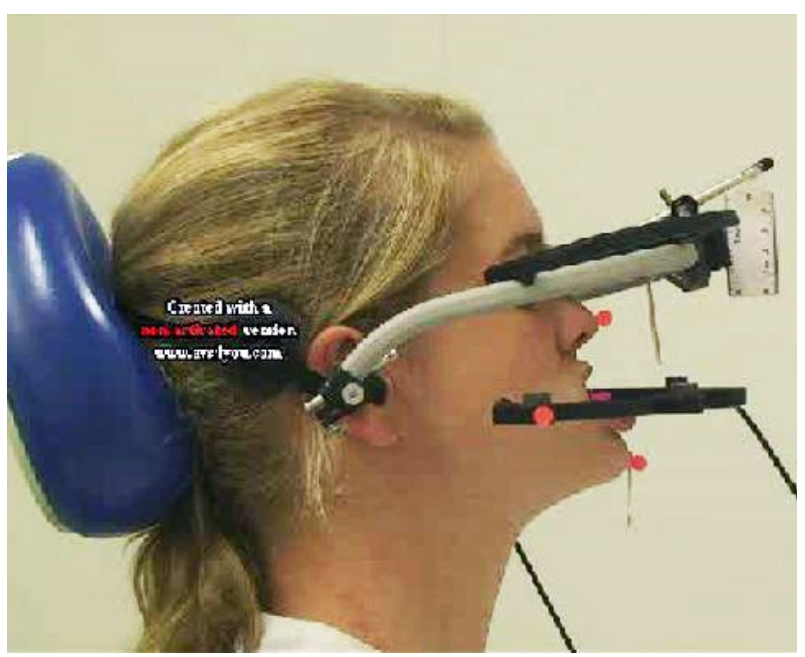

Abb. 45: Videoaufnahme nach dem Konvertieren ins .AVI-Format. 
Markergröße: Die durchgeführten Untersuchungen zeigen, dass die Größe der verwendeten Marker einen entscheidenden Einfluss auf die Messungen hat. Eine größere Punktfläche verursacht auch eine größere Abweichung im Analyseprogramm.

Die Software Rastreobackup erkennt Kontraste im Bild und kann einen Punkt, der sich von seiner Umgebung abhebt, erkennen und verfolgen. Ist der Kontrast aber zu schwach, kann das Programm den markierten Punkt im nächsten Bild verlieren und beispielsweise einem ähnlichen Punkt in der Umgebung folgen. Diese Funktionsweise kann auch in einem kleinen Bildausschnitt - wie dem Markerpunkt selbst - beobachtet werden. Die gelben Marker haben einen Durchmesser von $5 \mathrm{~mm}$, die orangenen sogar einen von $8 \mathrm{~mm}$. Innerhalb dieser Kreisfläche versucht das Programm zwar immer den gleichen Punkt zu treffen, durch den nahezu gleichen Kontrast aller Bildpunkte in der Kreisfläche, weicht die Software aber häufig auf einen nebenstehenden Punkt aus, der weiter verfolgt wird. Die Größe des Markers schafft also eine Varianz in sich.

Deshalb sollten für folgende Untersuchungen entweder kleinere Marker verwendet werden oder eine Markierung des Mittelpunktes vorgenommen werden (zum Beispiel einen kleinen dunklen Punkt), um präzise Messergebnisse zu erhalten.

Ausrichtung der Videographen: Da es für den korrekten Vergleich der Kurven des elektronischen Gesichtsbogens und der Videoanalyse notwendig ist, dass die Graphen in der gleichen Ausrichtung vorliegen, mussten die Videoaufzeichnungen an die Gesichtsbogenaufnahmen angepasst werden. Zur korrekten Ausrichtung der Filmsequenzen wurde die obere Sensorenebene des ARCUS®digma als Richtwert herangezogen. Diese Ebene dient dem Gesichtsbogen als Kalibrierungsebene und liegt dem Kopf des Probanden fest an (Abb. 46; Bild 1, grüne Ebene). Der Unterkieferbogen (Abb. 46; rote Ebene) wird der Kalibrierungsebene (grün) zugeordnet und richtet sich in seinen Messungen nun immer nach dieser Ebene. Aus diesem Grund haben äußere Einwirkungen wie die Bewegung des Kopfes keinen Einfluss auf die Messergebnisse (Abb. 46; Bild 2).

Um eine einheitliche Ausrichtung der Graphen beider Methoden zu erhalten, mussten die Kurven der Videoanalyse ebenfalls an die Kalibrierungsebene des ARCUS $R$ digma angepasst werden. Im Falle der Videoanalyse fehlt hierfür allerdings der automatische Bezug zu dieser Ebene. Aus diesem Grund wurde die Ebene zu Beginn der Bewegung registriert 
(Abb. 47; Bild 1, blaue Ebene). Die meisten Probanden verändern aber während der Bewegungsaufnahme ihre Kopflage. Dies führt zur Lageänderung der Bezugsebene. Da diese im Falle der Videoanalyse nicht automatisch geortet werden kann, wie es beim elektronischen Gesichtsbogen geschieht, kann dies zu Fehlern in der Messung führen (Abb. 47, Bild 2).
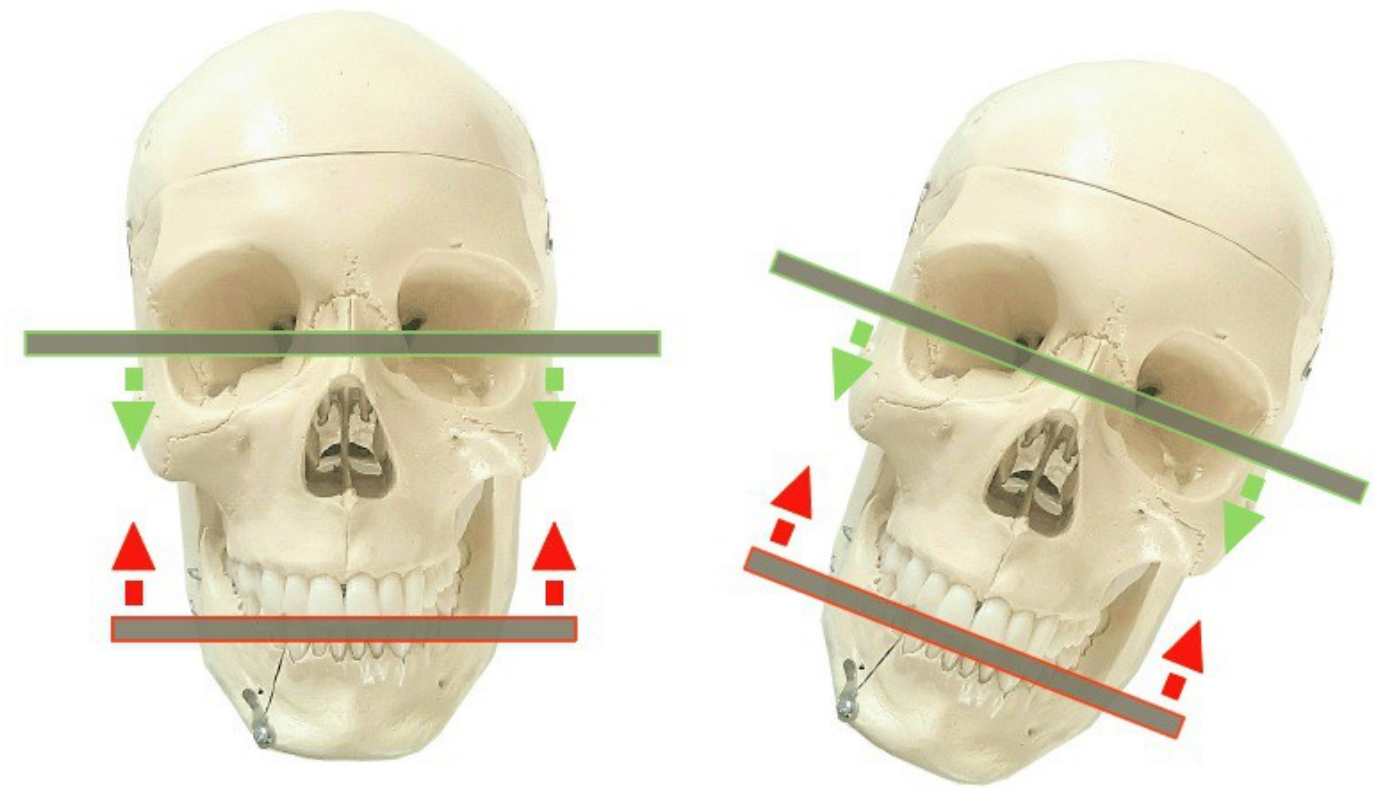

Abb. 46: Ausrichtung des Unterkieferbogens zur Kalibrierungsebene bei geradem und geneigtem Kopf beim elektronischen Gesichtsbogen.
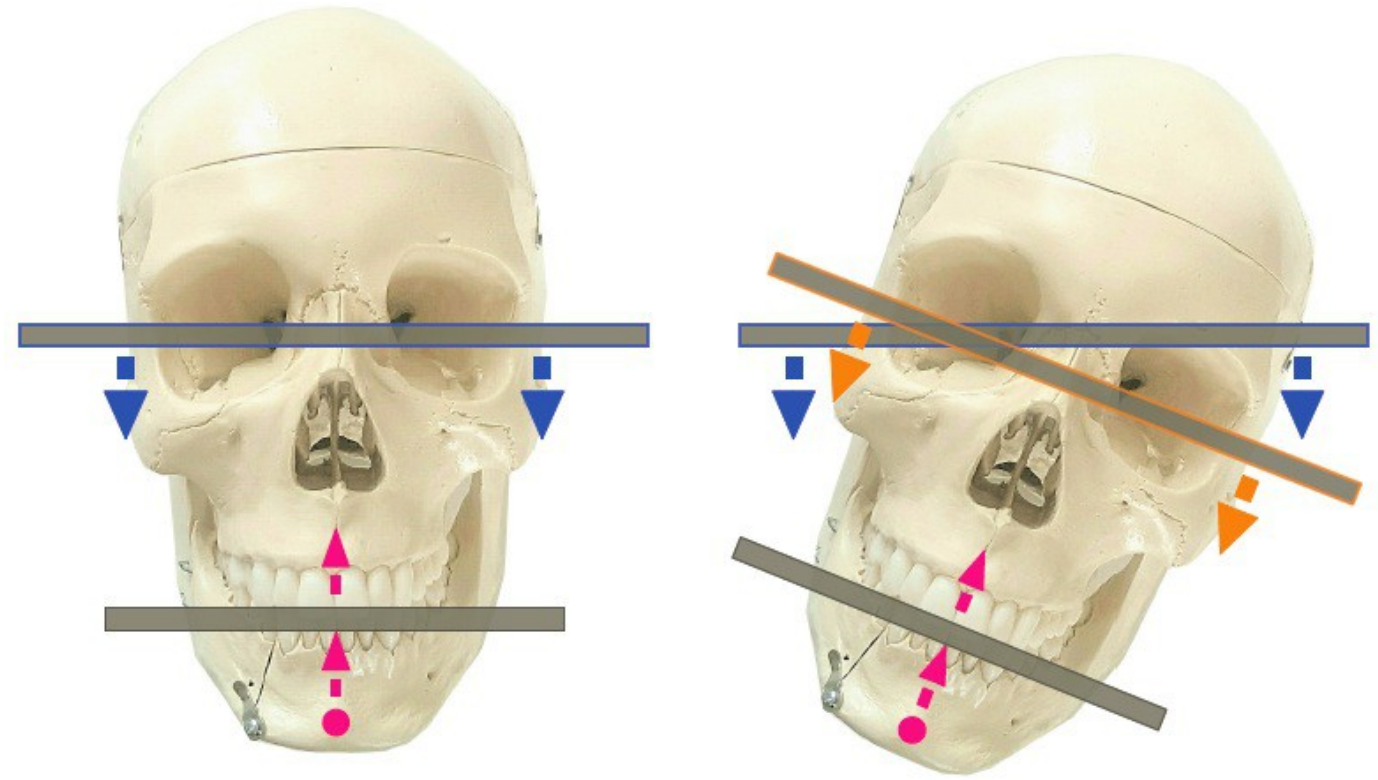

Abb. 47: Ausrichtung des Kinnmarkers zur Kalibrierungsebene des elektronischen Gesichtsbogens bei geradem und geneigtem Kopf. 
Die Bezugslinie müsste folglich in derselben Frequenz, in welcher das Programm Rastreobackup arbeitet, neu markiert werden, um für jeden Bewegungsmoment die korrekte Ausrichtung der Messpunkte zu erhalten (Abb. 47; Bild 2, orangefarbene Ebene).

Die Messung der Bewegung des Kopfes in der dritten Dimension ist mit den gegebenen Materialien nicht möglich.

Die Möglichkeit der korrekten Ausrichtung der Videoanalysegraphen hängt also stark mit den Aufnahmebedingungen zusammen. Idealerweise müsste der Kopf zusammen mit dem maxillären Bogen des ARCUS $R$ digma in einer Position fixiert werden, um Kopfbewegungen zu vermeiden und eine feste Referenzebene für die Ausrichtung der Videographen zu erhalten.

\subsubsection{Videoanalyse allgemein}

Markerpositionierung auf verschieblicher Oberhaut: Eine Besonderheit der Videografie ist es, dass die Referenzpunkte auf der Haut befestigt werden. Da das Weichgewebe über dem Knochen beweglich ist, gilt gleiches auch für die Messpunkte. Diese Tatsache wirft die Frage auf, ob eine Messung dieser Art ein korrektes Bild der Knochenbewegung wiedergeben kann, oder ob die Ergebnisse durch die Eigenbeweglichkeit der Messpunkte verfälscht werden.

1982 untersuchten Jemt und Hedegard, ob die Anbringung eines Markers auf der Kinnhaut zu Verfälschungen der Bewegung führt. Sie verglichen die Bewegungsbahnen des Kinnmarkers mit denen eines an den unteren Inzisiven angebrachten Markers und stellten fest, dass die Kinnreferenz für ihren untersuchten Kaurhythmus eingesetzt werden kann (Jemt und Hedegård 1982). Eine Studie von Atkinson und Shepherd führte 1955 zu dem Ergebnis, dass die Anbringung des Markers auf der Kinnhaut für die Unterkieferbewegungsmessung nicht geeignet ist (Atkinson und Shepherd 1955).

Die Ergebnisse der vorliegenden Untersuchung unterstützen die neueren Studienergebnisse von Jemt und Hedegard aus dem Jahr 1982. Mit der korrekten Markergröße und einer guten Bildauflösung können auch für die am Kinn angebrachten Messpunkte gute Ergebnisse erzielt werden. Kleine Messfehler durch die Verschiebung der Oberhaut kön- 
nen dabei jedoch nicht ausgeschlossen werden.

Erfassung der Kiefergelenkbewegungen: Ein weiterer Nachteil der Videoanalyse ist, dass die Bewegungen der Kiefergelenke mit den entsprechenden Winkelberechnungen nicht erfasst werden.

Eine Studie des Health Science Centers der Texas A\&M Universität hat ergeben, dass die zurückgelegte Strecke des Inzisalpunktes bei der Mundöffnung im Schnitt bei 48,6 mm lag, die zurückgelegte Strecke der Kondylen nur 11,9 mm betrug. Darüber hinaus ist eines der Ergebnisse der Studie, dass weder die gerade gemessene Distanz, noch die gemessene Rotation der Bewegung der Inzisivi mit der Bewegung der Kondylen übereinstimmt. Die Kondylen bewegen sich zum Beispiel weiter nach ventral als nach kaudal, während der Inzisalpunkt nach kaudal und posterior wandert. Aus diesem Grund liefert die Bewegung des Inzisalpunktes laut Studie keine aussagekräftigen Informationen und ist für diagnostische Zwecke bezüglich der Kondylenbewegung limitiert. Man sollte lieber die Bewegungsbahn der Kondylen zur Diagnose heranziehen (Travers et al. 2000).

Eine weitere Studie der Texas A\&M Universität kam zu dem Schluss, dass Protrusionsbewegung und Laterotrusionsbewegung eine mäßige Übereinstimmung mit der zugehörigen Kondylenbewegung aufweisen, eine direkte Messung der Kondylenbewegung aber zu besseren Messergebnissen kommt (Buschang et al. 2001).

Die Untersuchungen zeigen, dass die Ursache von CMD-Erkrankungen häufig im Kiefergelenk zu finden ist. Aus diesem Grund ist die Analyse der Kiefergelenkbewegung auch für die Videografie von großer Bedeutung.

Die Videoanalyse sollte deshalb durch einen Unterkieferbogen und eine entsprechende Kiefergelenkmessung ergänzt werden, um einen größeren Nutzen für die CMD-Diagnostik haben zu können.

Zeitlicher Aufwand: Ein weiteres wichtiges Ergebnis der Untersuchungen ist, dass der zeitliche Aufwand zur Generierung der Graphen höher ist, als zunächst angenommen. Zu Beginn der Arbeit wird die Videoanalyse als sehr einfache und schnelle Methode dargestellt. Jedoch zeigte sich im Verlauf der Auswertungen, dass insbesondere die Kurvenerstellung im Programm Rastreobackup viel Zeit in Anspruch nimmt. 
Vorteile der Videoanalyse: Die Videoanalyse ist eine nichtinvasive Methode, die für den Patienten ohne Einschränkungen in der Bewegung durchzuführen ist. In ihrem „Historical review of methods development" kommen Soboleva et al. zu dem Schluss, dass optoelektronische Geräte weniger störenden Einfluss auf die Unterkieferbewegung der Probanden nehmen, als vergleichbare Instrumente anderer Messsysteme (Soboleva et al. 2005b). Des Weiteren zeigt sich die Methode aufgrund ihrer geringen Ausstattung als kostengünstig.

\subsubsection{ARCUS $®$ digma}

Die Befestigung des Unterkieferbogens stellte sich bei einigen Probanden als problematisch heraus. Besonders bei Personen mit einem sehr tiefen Biss ist ein optimaler Halt schwer zu realisieren. Darüber hinaus hat sich der Bogen bei einigen Probanden im Laufe der Bewegungen gelockert und musste deshalb erneut angebracht werden. Es ist anzunehmen, dass die Ergebnisse der Messungen mit einem gelockerten Unterkieferbogen verfälscht sind.

Durch die Befestigung und das Gewicht des Unterkieferbogens ist es außerdem wahrscheinlich, dass die Unterkieferbewegungen beeinflusst werden. Dieser Ansicht sind auch Autoren anderer Studien (Pinheiro et al. 2011; Sadat-Khonsari et al. 2003). Ein weiterer Nachteil des Unterkieferbogens ist, dass dieser bei ruckartigen Bewegungen federt. Dadurch können Schwingungsartefakte in der Gelenkbahnaufzeichnung auftreten (Schierz und Reißmann 2008).

\subsubsection{Differenzen der beiden Methoden}

Der wohl wichtigste Diskussionspunkt der vorliegenden Arbeit ist die ungleiche Lokalisierung der Messpunkte.

Der ARCUS®digma-Messpunkt liegt, je nach Positionierung des paraokklusalen Löffels, knapp kaudal des Unterkiefer-Inzisalpunktes, die Videoanalysemessung erfolgt am Weichteilpogonion. Dieser Unterschied spiegelt sich deutlich in den Graphen der Bewegungen wider. 
Für die Diskussion der Messpunktlokalisation beziehungsweise der daraus resultierenden Kurvenabweichungen wird an dieser Stelle auf die Diskussion der Ergebnisse (4.3.2 Mittelwerte der Differenzen) verwiesen.

\subsubsection{Kostenübersicht}

An dieser Stelle soll auf die Kosten der beiden untersuchten Messmethoden eingegangen werden.

Die Übersicht der Beträge zeigt, dass die Videoanalyse mit einem Preis von etwa 475,00 $€$ deutlich unter dem ARCUS $R$ digma mit einem Preis von rund 8.300,00 € liegt (Tab. 5). Dieses Ergebnis zeigt, dass die Anforderung an die neue Messmethode bezüglich der Kostenminimierung erfüllt wurde.

Die Benutzung der Software war in der vorliegenden Untersuchung ohne Ausgaben verbunden. Bei einer Weiterentwicklung der Programme ist aber davon auszugehen, dass ihre Anschaffung mit einem Kostenaufwand verbunden sein wird.

Die Preise der Videoanalysematerialien entsprechen hier möglichen Anschaffungsbeträgen und sind je nach Wahl des jeweiligen Gerätes variabel.

\begin{tabular}{|c|c|c|c|}
\hline & \multirow{6}{*}{$\begin{aligned} \text { ARCUS®digma }(€) \\
\\
\\
\\
\sim 8.300,00\end{aligned}$} & \multicolumn{2}{|l|}{ Videoanalyse $(€)$} \\
\hline & & HD-Kamera ab & 150,00 \\
\hline & & Stativ ab & 15,00 \\
\hline & & Markermaterial & 10,00 \\
\hline & & Laptop ab & 300,00 \\
\hline & & Software für diese Studie & 0,00 \\
\hline Gesamtpreis & $\sim 8.300,00$ & Für diese Studie & $\sim 475,00$ \\
\hline
\end{tabular}

Tab. 5: Kostenübersicht. 


\subsection{Diskussion der Ergebnisse}

\subsubsection{Bewegungsmaxima}

Mittelwerte allgemein: Für den elektronischen Gesichtsbogen betragen die durchschnittlichen Maxima-Werte: Laterotrusion links $8,21 \mathrm{~mm}$, Laterotrusion rechts $7,4 \mathrm{~mm}$, frontale Mundöffnung 49,26 mm, sagittale Mundöffnung 47,23 mm und Protrusion 9,6 mm.

Die Mittelwerte der Videografie lauten: Laterotrusion links 14,04 mm, Laterotrusion rechts 13,33 mm, frontale Mundöffnung 47,23 mm, sagittale Mundöffnung 51,78 mm und Protrusion $11 \mathrm{~mm}$.

\begin{tabular}{|l|l|l|l|l|l|}
\hline Bewegung & $\begin{array}{l}\text { Laterotrusion } \\
\text { links }\end{array}$ & $\begin{array}{l}\text { Laterotrusion } \\
\text { rechts }\end{array}$ & $\begin{array}{l}\text { frontale } \\
\text { Mundöffnung }\end{array}$ & $\begin{array}{l}\text { sagittale } \\
\text { Mundöffnung }\end{array}$ & Protrusion \\
\hline ARCUS@digma & $8,21 \mathrm{~mm}$ & $7,4 \mathrm{~mm}$ & $49,26 \mathrm{~mm}$ & $47,23 \mathrm{~mm}$ & $9,6 \mathrm{~mm}$ \\
\hline Videoanalyse & $14,04 \mathrm{~mm}$ & $13,33 \mathrm{~mm}$ & $47,23 \mathrm{~mm}$ & $51,78 \mathrm{~mm}$ & $11 \mathrm{~mm}$ \\
\hline
\end{tabular}

\begin{tabular}{|l|c|c|c|c|c|}
\hline Krogh-Poulsen & $9-12 \mathrm{~mm}$ & $9-12 \mathrm{~mm}$ & - & $40 \mathrm{~mm}$ & - \\
\hline J.R. Strub et al. & $7-12 \mathrm{~mm}$ & $7-12 \mathrm{~mm}$ & - & $40-60 \mathrm{~mm}$ & $7-11 \mathrm{~mm}$ \\
\hline K.M. Lehmann & - & - & - & $40-50 \mathrm{~mm}$ & $9-10 \mathrm{~mm}$ \\
\hline $\begin{array}{l}\text { Studie der Cam- } \\
\text { peche Universität }\end{array}$ & - & - & - & $46,61 \mathrm{~mm}$ & - \\
\hline $\begin{array}{l}\text { Siebert } \\
\text { Studie der Texas } \\
\text { A\&M University }\end{array}$ & $11,0 \mathrm{~mm}$ & $11,5 \mathrm{~mm}$ & - & $\begin{array}{l}\text { §: } 37-60 \mathrm{~mm} \\
0: 37-53 \mathrm{~mm}\end{array}$ & - \\
\hline
\end{tabular}

Tab. 6: Mittelwerte der einzelnen Bewegungen für die Analyseverfahren des elektronischen Gesichtsbogens und der Videoanalyse.

In der Literatur sind unterschiedliche Werte für die Grenzbewegungen des Unterkiefers zu finden:

Für die maximale Mundöffnung betragen die Werte nach Krogh-Poulsen 40 mm (KroghPoulsen 1973). Von J.R. Strub wird ein durchschnittlicher Wert von 40 - 60 mm angegeben 
und nach K.M. Lehmann beträgt die maximale Öffnung 40 - 50 mm (Lehmann et al. 2009; Strub et al. 2011). Darüber hinaus wurde in einer Studie der Campeche Universität an 254 gesunden Probanden eine durchschnittliche Mundöffnung von 46,61 mm ermittelt. Die Ergebnisse lagen bei Werten von $42,2 \mathrm{~mm}$ bis $49,1 \mathrm{~mm}$, wobei die maximale Mundöffnung bei Frauen im Schnitt 3,29 mm unter der der Männer lag (Casanova-Rosado et al. 2012). Die Normwerte nach Siebert (mit addiertem Overbite) liegen für Männer bei 37 - $60 \mathrm{~mm}$ und für Frauen bei 37 - 53 mm (Siebert 1987).

In den meisten Studien wird die Messmethodik zur Bestimmung der maximalen Mundöffnung nicht angegeben. Die Messung kann entweder nur anhand der Schneidekantendistanz erfolgen, oder den Overbite miteinbeziehen (Schneidekantendistanz + Overbite). Die Werte können also um wenige Millimeter variieren.

Für die Laterotrusion betragen die Werte nach Krogh-Poulsen 9 - 12 mm und nach J.R. Strub 7 - 12 mm (Krogh-Poulsen 1973; Strub et al. 2011). Eine Studie der Texas A\&M University mit 27 Probanden ergab eine durchschnittliche Laterotrusion nach rechts von 11,5 mm und eine Laterotrusion nach links von 11,0 mm (Buschang et al. 2001).

Die maximale Protrusion beträgt nach Strub 7 - $11 \mathrm{~mm}$, nach Lehmann 9 - $10 \mathrm{~mm}$ und die Studie der Texas A\&M University ergab eine mittlere Protrusion von 11,5 mm (Buschang et al. 2001; Lehmann et al. 2009; Strub et al. 2011).

Es lässt sich zusammenfassend sagen, wie auch aus der Tabelle 6 ersichtlich ist, dass die Ergebnisse der vorliegenden Studie für die Mittelwerte der Grenzbewegungen im Wesentlichen mit den Ergebnissen anderer Studien übereinstimmen.

Streuung der Minimal-Maximal-Werte: Bei der Betrachtung der Graphen fällt auf, dass die Minimal- und Maximalwerte einzelner Probanden zum Teil weit auseinander liegen. Trifft diese Divergenz sowohl auf die Gesichtsbogenwerte als auch auf die Videografiewerte zu, so ist davon auszugehen, dass der Proband die fünf Wiederholungen der Grenzbewegungen nicht reproduzierbar durchgeführt hat. Die Bewegungen wurden also tatsächlich zum Teil kleiner und zum Teil größer ausgeführt. Bei einigen Personen liegen die großen Abstände zwischen den Minimal- und Maximalwerten aber nur für die Videoanalyse vor. In diesen Fällen ist eine Messungenauigkeit in der Videoauswertung wahrscheinlich. 
Diese Ungenauigkeit kann verschiedene Ursachen haben: Sie kann der Qualitätseinbuße, welche durch das Konvertieren der Videos aus dem .MTS-Format in das .AVI-Format eingetreten ist, geschuldet sein. Darüber hinaus kann sie durch die Größe der Marker und die nicht eindeutige Verfolgung der markierten Punkte im Programm Rastreobackup entstanden sein. Eine weitere Ursache könnte die Überlagerung des Kinnpunktes durch den Unterkiefergesichtsbogen und dessen Kabel sein.

Es ist davon auszugehen, dass sich die Kombination dieser Faktoren auf die Ergebnisse der Videografiemessungen ausgewirkt hat.

Größe der Messwerte: Die Graphen zeigen, dass die Mediane des ARCUS®digma und die der Videografie zum Teil deutlich voneinander abweichen. Bei den Graphen für die Laterotrusion nach links sowie nach rechts ist zu erkennen, dass die intraindividuellen Mediane der Videoanalysedaten durchgängig über denen des elektronischen Gesichtsbogens liegen. Für die aus frontaler Sicht betrachtete Mundöffnung liegen 20 von 32 Probandenwerten des ARCUS®digma über den Videografiewerten. In den Grafiken für die Mundöffnung in der sagittalen Ebene und für die Protrusion liegen die Werte der Videoaufnahme mit 25 von 32 beziehungsweise 26 von 32 Werten im Durchschnitt über den ARCUS $®$ digma-Mittelwerten.

Um eine genaue Aussage bezüglich der Abweichungen treffen zu können, wurden die Mittelwerte der Differenzen gebildet.

\subsubsection{Mittelwerte der Differenzen}

Die Ergebnisse machen deutlich, dass die Maxima-Werte der Videografie für die Bewegungen Laterotrusion links, Laterotrusion rechts, Mundöffnung-sagittal und Protrusion im Durchschnitt größer sind, als die Werte des Gesichtsbogens. Die Werte für Mundöffnung-frontal sind hingegen im Durchschnitt kleiner, als die des ARCUS®digma.

Die Ursache der ungleichen Ergebnisse kann mit der unterschiedlichen Positionierung der Messpunkte begründet werden. Beim elektronischen Gesichtsbogen liegt der Unterkiefermesspunkt, je nach Positionierung des paraokklusalen Löffels, einige Millimeter kaudal des Inzisalpunktes. Der Marker für die Videoanalyse ist hingegen auf dem Kinn ange- 
bracht. Die Messpunkte der beiden zu vergleichenden Systeme sind also nicht deckungsgleich.

Bei der Bewegung des Unterkiefers nach rechts oder links (Laterotrusion) tritt neben der Translationsbewegung des Kiefers zur jeweiligen Seite hin auch eine Rotationsbewegung auf. Der Winkel zwischen den Bewegungsbahnen des reinen Vorschubes und der Bewegung des schwingenden Kondylus (Mediotrusionsseite) wird Bennettwinkel genannt und

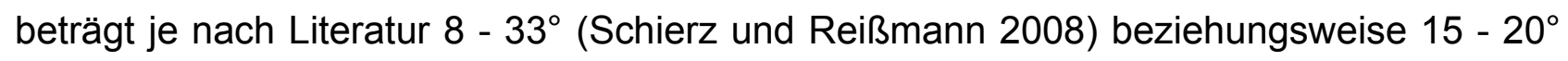
(Lehmann et al 2009). Der Unterkiefer rotiert also nach rechts beziehungsweise links (Schierz und Reißmann 2008). Das Weichteilpogonion (am weitesten anterior gelegener Punkt des Kinns in der Medianebene) liegt weiter vom Drehzentrum entfernt als der Inzisalpunkt und befindet sich somit auf einer größeren Kreisbahn. Dadurch bewegt sich dieser Punkt bei der Laterotrusion und der damit einhergehenden Rotation des Unterkiefers weiter nach lateral (Abb. 48). Bei der Mundöffnung kommt es zu dem gleichen Effekt. Hier verschiebt sich der Kinnpunkt weiter nach kaudal als der Inzisalpunkt (Abb. 49).

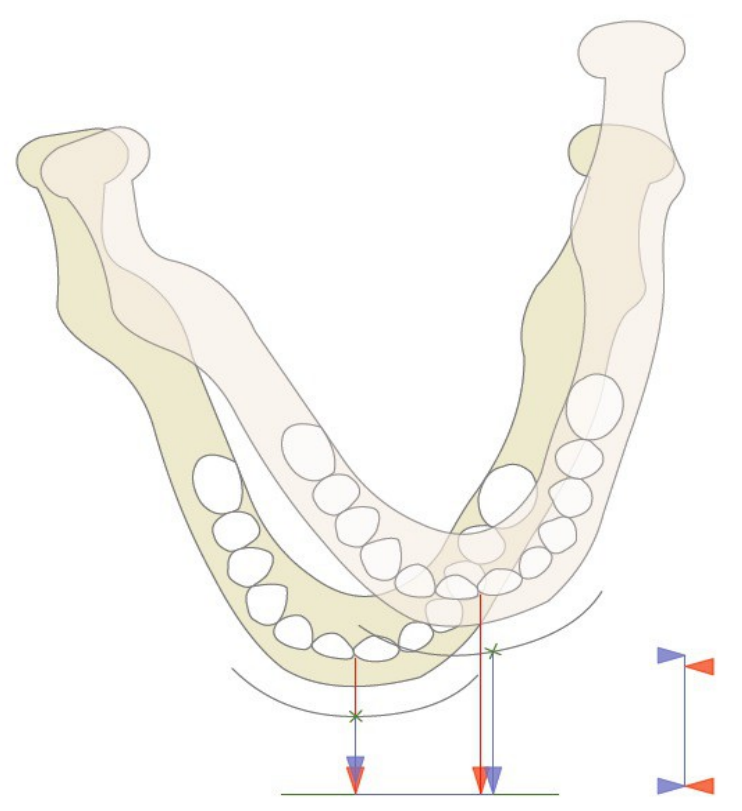

Abb. 48: Rotation des Unterkiefers bei der Laterotrusionsbewegung. Der Kinnpunkt verschiebt sich weiter nach lateral, als der Inzisalpunkt.

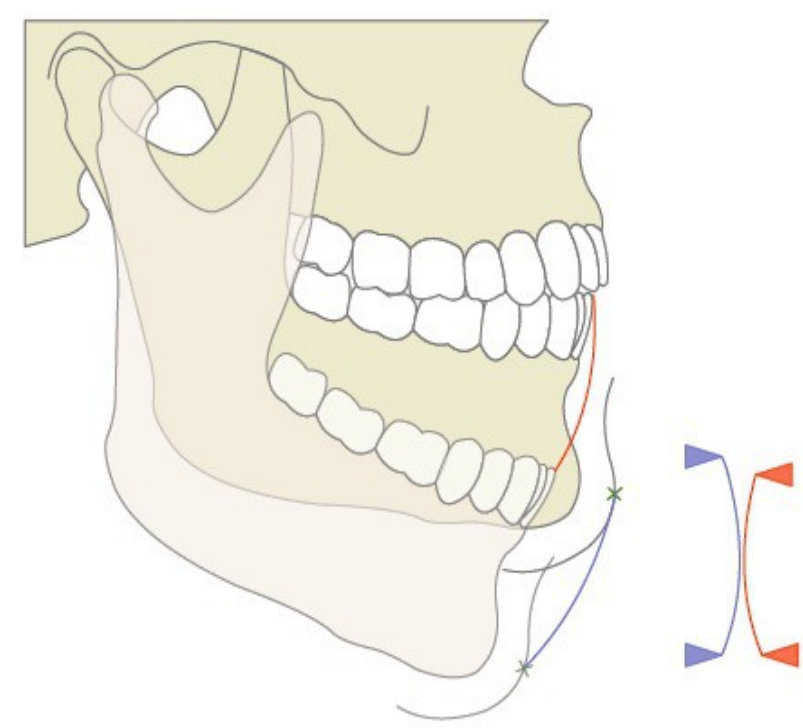

Abb. 49: Die Mundöffnung von sagittal betrachtet. Die Bewegungsbahn des Kinnpunktes ist länger, als die des Inzisalpunktes. 
Auf die Frontalebene projiziert, muss die Mundöffnung jedoch gesondert betrachtet werden. Durch die Verschiebung des Rotationszentrums bei der Mundöffnung nach ventral und kaudal und die individuelle Positionierung der Messpunkte auf ihrer Kreisbahn, wird der Kinnpunkt stark nach dorsal verschoben. Der Inzisalpunkt verschiebt sich hingegen überwiegend nach kaudal und kaum nach dorsal.

Die Bewegung des Unterkiefers in der dritten Dimension kann aus der frontalen Ebene nicht gemessen werden, was dazu führt, dass die Dorsalbewegung der Messpunkte nicht berücksichtigt wird. Dies hat vorwiegend Auswirkungen auf die Darstellung der Bewegungsbahn des Kinnpunktes, die durch die Projektion auf die Frontalebene nicht nur nicht vergrößert abgebildet wird, sondern sogar verkürzt erscheint (Abb. 50).

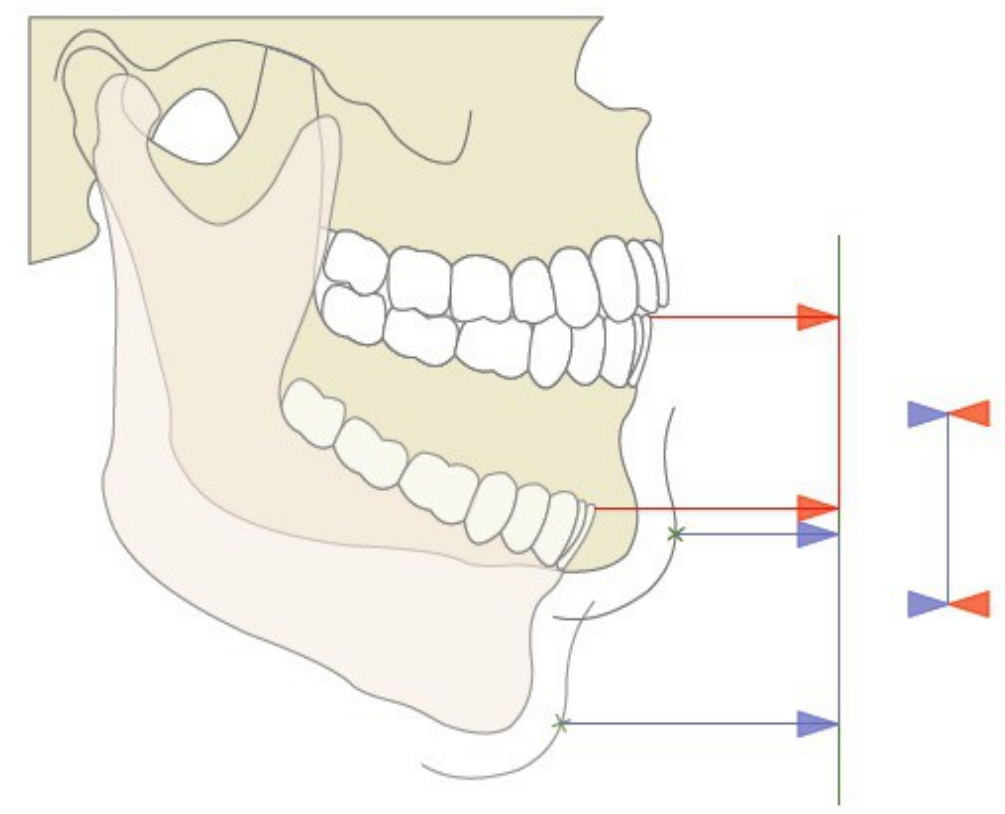

Abb. 50: Projektion der Mundöffnung auf die Frontalebene. Die Bewegungsbahn des Kinnpunktes bildet sich verkürzt ab.

Dieses Phänomen wird besonders durch die Mittelwerte der Differenzen der einzelnen Bewegungen veranschaulicht (Tab. 7). Die Mittelwerte für die Laterotrusion nach links beziehungsweise rechts und für die Mundöffnung-sagittal sind deutlich im negativen Bereich, was auf eine Vergrößerung der Videoanalysewerte hindeutet. Der Wert für die Protrusion ist nur leicht negativ, was damit zu erklären ist, dass diese Bewegung durch die Gleitbewe- 
gung des Kondylus auf dem Tuberculum articulare vorwiegend aus einer Translationsbewegung besteht und die zusätzliche Rotationsbewegung wenig Einfluss hat.

Das Ergebnis der frontalen Mundöffnung zeigt den erwarteten positiven Mittelwert. Hier erscheint die Bewegung des Kinnpunktes durchschnittlich verkleinert.

\begin{tabular}{|l|r|}
\hline & Mittelwerte \\
\hline Laterotrusion links & $-5,82881$ \\
\hline Laterotrusion rechts & $-5,94794$ \\
\hline Mundöffnung frontal & 2,02686 \\
\hline Mundöffnung sagittal & $-4,49607$ \\
\hline Protrusion & $-1,38074$ \\
\hline
\end{tabular}

Tab. 7: Mittelwerte der Differenzen.

\subsubsection{Vergleichsberechnung}

Die Berechnung sollte ermitteln, ob die Abweichungen der Messergebnisse der beiden Methoden mit der unterschiedlichen Lokalisation ihrer Messpunkte zu erklären ist.

Die Ergebnisse des Vergleichs der Videoanalysemediane mit den Werten der Faktorenrechnung zeigen, dass sich die theoretisch ermittelten Daten den Tatsächlichen annähern. Bei einigen Probanden liegen diese und die intraindividuellen Mediane aber auch auseinander. Es ist sogar zu beobachten, dass die Werte der Faktorberechnung weiter von den tatsächlichen Daten entfernt liegen, als die ursprünglichen ARCUS®digma-Mediane.

Diese Abweichungen sind dadurch zu erklären, dass es sich bei dem ermittelten Faktor um einen Durchschnittswert handelt. Die individuellen anatomischen Gegebenheiten bestimmen den sagittalen Abstand zwischen Inzisal- und Kinnpunkt. Da der Kinnpunkt bei einem größeren Abstand in Relation zum Inzisalpunkt weiter nach außen schwenkt als bei einem kleineren Abstand, muss auch der Umrechnungsfaktor größer gewählt sein. Bei einem kleinen sagittalen Abstand, muss auch der Umrechnungsfaktor kleiner sein.

Bei den Probanden mit einem überdurchschnittlich großen Messpunkteabstand reicht der durchschnittliche Faktor also nicht aus. Gleiches gilt für den Probanden mit unterdurchschnittlichem Inzisal-Kinn-Abstand. Hier liegen die tatsächlichen ARCUS®digma- und Vi- 
deoanalysewerte schon nah beieinander. Durch die Faktormultiplikation liegt der theoretisch ermittelte Wert nun über dem tatsächlichen Videografie-Median.

Bei der Betrachtung der unterschiedlichen Kinnprominenzen, die die Ergebnisse der Videografie sehr variabel erscheinen lassen, soll hier darauf hingewiesen werden, dass auch die Inzisalpunkte diesen Schwankungen unterliegen. Je nach Ausprägung der Protrusion und Retrusion der Unterkieferinzisivi und je nach Anbringung des paraokklusalen Löffels, werden sich die Messergebnisse voneinander unterscheiden.

Die theoretischen Berechnungen haben ergeben, dass der durchschnittliche Abstand zwischen den Messpunkten etwa $3 \mathrm{~cm}$ beträgt (Abb. 52). Ob dieser Wert tatsächlich mit den durchschnittlichen Werten der Probanden übereinstimmt, ist auf Grundlage der Untersuchungen nicht eindeutig feststellbar. In einer weiterführenden Studie könnten die individuellen anatomischen Gegebenheiten der Patienten anhand von CT-Bildern untersucht und die Ergebnisse so auf ihre Korrektheit überprüft werden.

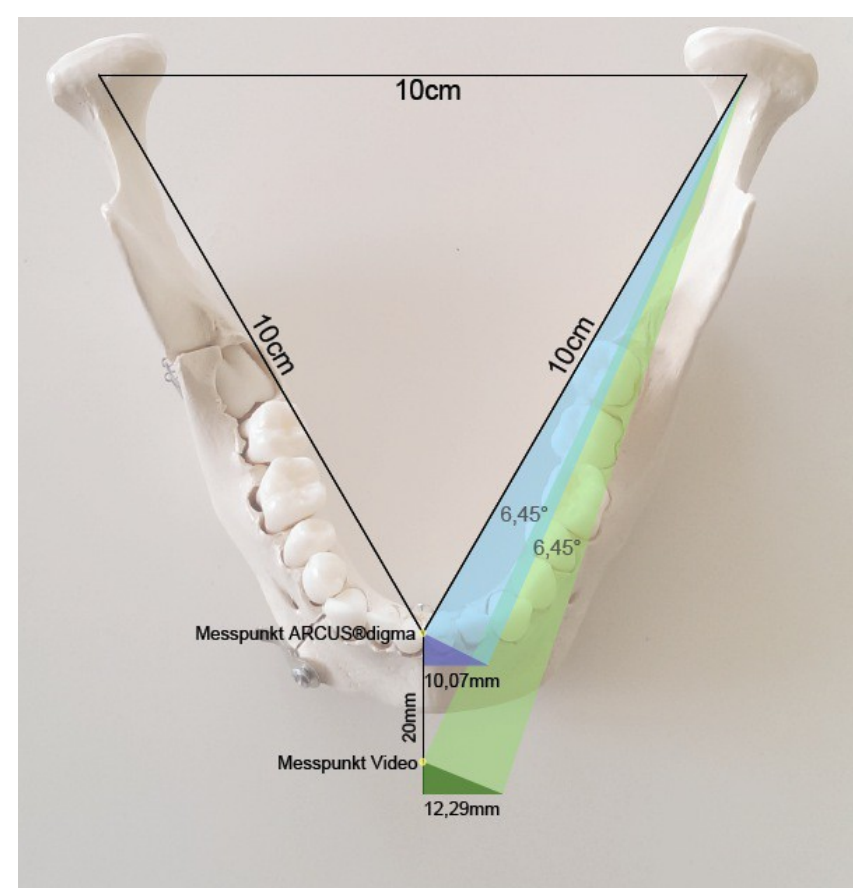

Abb. 51: Grafische Darstellung der Vergleichsberechnung bei einem Messpunktabstand von $2 \mathrm{~cm}$.

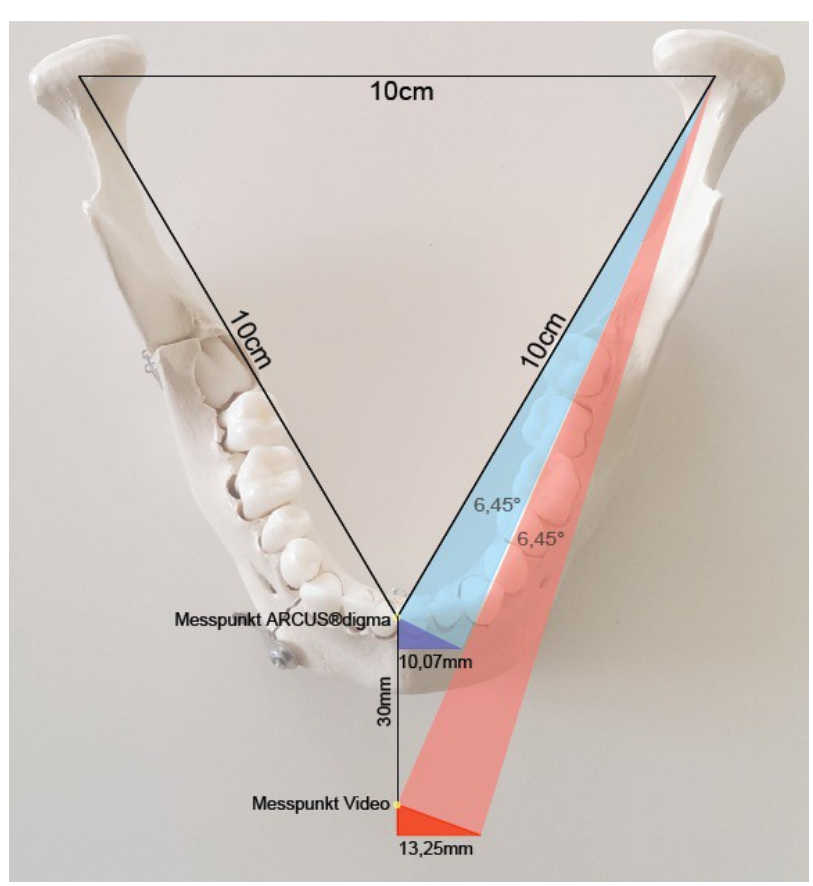

Abb. 52: Grafische Darstellung der Vergleichsberechnung bei einem Messpunktabstand von $3 \mathrm{~cm}$. 


\subsubsection{Bewertungskriterien}

In der Analyse der Bewertungskriterien wurden die Graphen der beiden Methoden in Bezug auf ihre Form verglichen. Dieser Vergleich wurde während der Versuchsdurchführung mit in die Untersuchungen aufgenommen, da sich herausstellte, dass die Überprüfung, ob ein Analysesystem die Bewegungsbahn in ihrer Form korrekt wiedergibt, von grundlegender Bedeutung für die CMD-Diagnostik ist. Denn die meisten CMD-Symptome können in erster Linie auf Grundlage der Bewegungen zwischen den Maximalstellungen beurteilt werden.

Dieses Untersuchungsergebnis unterstützt die Ergebnisse vorangegangener Studien. In diesen weisen einige Autoren darauf hin, dass die Maxima der Grenzbewegungen keine relevanten Ergebnisse hinsichtlich der CMD-Diagnose hervorbringen.

So gibt es nach Szentpétery keine allgemeingültigen Werte für die Bewegungsmaxima. Nach Meinung des Autors sei es vielmehr so, dass die Angaben der Patienten, ob sich die Mundöffnung normal oder eingeschränkt anfühlt, wichtiger und zuverlässiger sind, als festgelegte Grenzwerte (Szentpétery 1993). Darüber hinaus ist es nach Travers so, dass durch die Dehnung der Muskeln und Bänder bei wiederholter Bewegung die Maximalwerte größer werden (Travers et al. 2000).

Hinsichtlich des Nutzens für die CMD-Diagnostik kommt eine Studie aus Marburg mit 600 Probanden, in der die Bewegungen Öffnung, Protrusion und rechte sowie linke Laterotrusion untersucht wurden, zu dem Ergebnis, dass keine Unterschiede in der Unterkiefermobilität zwischen gesunden Probanden und Probanden mit einer Funktionsstörung bestehen. Es konnten nur Unterschiede zwischen den einzelnen Altersgruppen, sowie zwischen den Geschlechtern festgestellt werden (Szentpétery 1993). Eine Studie aus Zagreb kommt ebenfalls zu dem Ergebnis, dass die Länge der Bewegung des Unterkiefers und auch der Kondylen kein zuverlässiger Indikator ist, um die Funktion des temporomandibularen Systems zu bestimmen (Kralijevic et al. 2003).

Deshalb ist es nach Szentpétery fraglich, ob die Maximalwerte der Unterkieferbewegung zur Diagnose einer CMD beitragen können (Szentpétery 1993). Vielmehr ist es so, dass aus Länge, Form und Symmetrie der Aufzeichnungsspuren indirekt auf zugrunde liegende Krankheitsbilder geschlossen werden kann (Schierz und Reißmann 2008).

Die aufgeführten Studien machen deutlich, dass die Form der Bewegungsbahnen wesent- 
liche Schlüsse auf die Erkrankungsursache zulässt. Aus diesem Grund wurde diese Analyse in die vorliegenden Untersuchungen aufgenommen und die Graphen bezüglich ihrer Form verglichen.

Die anhand von Bewertungskriterien durchgeführten Analysen der einzelnen Bewegungsabschnitte zeigen Übereinstimmungen von 51 \% (Closing endpoints - Posselt sagittal) bis $96 \%$ (Closing - Posselt sagittal). Insgesamt entsprechen sich die Graphen der beiden Methoden zu $80 \%$.

Bei der Betrachtung der Ergebnisse fällt auf, dass bestimmte Bewegungsabläufe eine besonders große und andere eine eher kleine prozentuale Übereinstimmung aufweisen.

Insbesondere die Bewegungsabschnitte left closing, right closing, opening endpoint der frontalen Grenzbewegungen und der closing endpoint aus dem Posselt sagittal weisen mit knapp über 50 \% keine eindeutige Messübereinstimmung auf. Bei den Bereichen opening endpoint (frontale Grenzbewegung) und closing endpoint (sagittale Grenzbewegung) ist auf die Sensibilität dieser Punkte hinzuweisen. Schon bei kleinen Bewegungen des Kopfes, verändert sich die Bewegungsbahn so, dass sie nicht mehr in einem Punkt geschlossen wird. Betrachtet man die anderen Bewegungssequenzen, so fällt auf, dass auch bei der Verschiebung der Bewegungskurve einer Messmethode, die Form der Bewegung weiterhin übereinstimmt. Somit stimmt der Endpunkt der Bewegung bei einer Methode vielleicht nicht mehr mit dem Ausgangspunkt überein, die Bewegung „laterotrusion left“ wird jedoch beispielsweise weiterhin für beide Messmethoden als gleichförmig angesehen.

Diese Erklärung lässt sich so zusammenfassen, dass die verschiedenen Bewegungsabschnitte eine unterschiedliche Anfälligkeit für Messungenauigkeiten aufweisen.

Da die Bewertung der Graphen keiner mathematischen Genauigkeit unterlag und diese nur durch Betrachtung ausgewertet wurden, sind die Ergebnisse nicht als methodisch fundierte Werte anzusehen. Dennoch ist eine deutliche Tendenz zur grundsätzlichen Übereinstimmung erkennbar.

Zusammenfassend lässt sich sagen, dass die korrekte Wiedergabe der Form der Unterkieferbewegung von großer Relevanz für die CMD-Diagnostik ist. Um grundsätzlich beurteilen zu können, ob dies für die Videoanalyse zutrifft, wäre der Vergleich mit anderen Stu- 
dienergebnissen wünschenswert.

Da es sich bei der vorliegenden Untersuchung um die erste vergleichende Studie dieser Art handelt, können den Ergebnissen leider keine entsprechenden Resultate anderen Studien gegenübergestellt werden.

\subsection{Ausblick}

\subsubsection{Aktuelle Forschung}

Das Fundament für die verbesserte Diagnosemöglichkeit verschiedener Kiefergelenkerkrankungen gliedert sich in zwei Bereiche. Der erste beschäftigt sich mit der Erforschung des Kiefergelenks und des nozizeptiven Systems, der zweite befasst sich mit der Entwicklung neuer Techniken, um Formen und Bewegungen des Unterkiefers präzise wiedergeben zu können. Die hohe Anzahl der Publikationen zu diesem Thema zeigt, dass die CMD ein brisantes Thema in der zeitgenössischen Zahnmedizin darstellt.

In der jüngsten Forschung werden viele Fragen aufgeworfen: Wie sieht die genaue Anordnung und Struktur der Kollagene und GAG im Kiefergelenk aus? Welche Wachstumsfaktoren haben Einfluss auf die Zellen des Diskus? Welche Materialien sind biokompatibel und ähnlich mechanisch belastbar (Detamore und Alhanasiou 2003)? In wieweit sind Genstrukturen an der Schmerzübertragung beteiligt (Zubieta et al. 2003)?

In die CMD-Wissenschaft werden periphere Regionen des Körpers einbezogen. In einer Studie von Lobbezoo weist dieser darauf hin, dass das zentrale nozizeptive System weitere Antworten für die Probleme der CMD-Erkrankung liefern könnte. Es könnte zukünftig möglich sein, hochauflösende bildgebende Verfahren zu benutzen, um zentrale nozizeptive Prozesse und periphere Aktivitäten zu untersuchen, um mehr über die Probleme im Kiefergelenk zu erfahren (Lobbezoo et al. 2004). Das Kiefergelenk mit seinen Umbauprozessen und seiner Innervation muss also auf molekularer Ebene weiter untersucht werden (Lobbezoo et al. 2004).

Für die Bewegungsanalysen des Unterkiefers kann heute fast alles in die digitale Welt übersetzt werden. Mit Hilfe von bildgebenden Verfahren lassen sich Unterkiefer und Kiefergelenke dreidimensional darstellen. 
Es gibt verschiedene Verfahren wie optoelektronische Scanner (Optocom), Laserscanner, intraorale Videokameras oder CT-Bilder, mit denen man virtuelle 3D-Kiefermodelle erstellen kann (Röhrle et al. 2009). In einer Studie der Wright State University wurden Unterkiefer virtuell rekonstruiert (Ingawalé und Goswami 2012). In einer weiteren Studie wurden die Modelle der Ober- und Unterkiefer eingescannt, um die Bewegung der Dentition gegeneinander am Computer zu rekonstruieren (Fang und Kuo 2008). Sind patientenbezogene Bewegungsmessungen vorhanden, kann mit Hilfe der dreidimensionalen Kiefermodelle und der Bewegungsaufzeichnung ein virtueller Artikulator erstellt werden (Fang und Kuo 2008).

In einer Stuttgarter Studie sind die Forscher auf ähnliche Art und Weise vorgegangen. Mit einem fest installierten optoelektronischen System mit acht Kameras (VICON MX; am Biomechanischen Labor in Auckland) wurden Bewegungsmessungen vorgenommen. Mittels CT-Bildern wurden virtuelle Kiefermodelle konstruiert und mit Hilfe der Bewegungsaufzeichnung ein virtueller Artikulator entwickelt (Röhrle et al. 2009). Dieser soll mit einer dentalen CAD/CAM-Software gekoppelt werden, um einen sowohl in statischer als auch dynamischer Okklusion perfekt angepassten Zahnersatz zu fertigen.

Diese Verfahren verkürzen nicht nur den Zeitraum der Herstellung des Zahnersatzes, sondern minimieren auch das Einschleifen nach der Eingliederung.

Im Folgenden wird gezeigt, dass es neben der reinen Analyse der Unterkieferbewegungskurven auch andere Ansätze zur Diagnose einer CMD gibt.

Eine Studie von Sadat-Khonsari beschäftigt sich mit der Bahn der momentanen Drehzentren (ICR: instantaneous centers of rotation) des Unterkiefers. Für Patienten ohne Funktionsstörung ist die ICR-Bahn durch einen harmonischen Verlauf mit kondylennahem Beginn gekennzeichnet. Die Rotationszentren bewegen sich daraufhin nach dorsokaudal, bei weiterer Kieferöffnung nach ventral und schließlich nach ventrokranial. Die ICR-Bahn erkrankter Gelenke zeigt hingegen einen irregulären sternförmigen Verlauf mit spontanen Richtungsänderungen. Schon kleinste Bewegungen führen zu großen Änderungen des Rotationszentrums. Sadat-Khonsari spricht von einem Vergrößerungseffekt, der jedoch auch anfällig gegenüber Registrierfehlern ist (Sadat-Khonsari et al. 2003).

Darüber hinaus kann auch der zeitliche Faktor einer Bewegung zum genaueren Verständnis beitragen. In einer japanischen Arbeit wurde nicht nur die Bewegung des Unterkiefers 
aufgezeichnet, sondern auch die Beschleunigung dieser Bewegungen gemessen. Das Forschungsteam kam zu dem Ergebnis, dass durch die Geschwindigkeitsänderungen schon kleine CMD-Symptome erfasst werden können, wie zum Beispiel eine Diskusverlagerung (Koseki et al. 2007). Ist die Bewegung durch den verlagerten Discus articularis blockiert, verlangsamt sie sich. In dem Moment, in welchem der Discus wieder auf den Kondylus zurückspringt, kommt es zur Beschleunigung. In einer weiteren Studie wurden ebenfalls die Beschleunigungen für die Bewegungsanalyse berechnet (Röhrle et al. 2009). Die Literatur zeigt, dass die Wissenschaft auf vielen Ebenen arbeitet und weite, zum Teil periphere Bereiche (z.B. auf molekularer Ebene) bedient. Die hier vorliegende Untersuchung zeigt, in Anlehnung an die technische Forschung im Allgemeinen, dass die Anwendung der Geräte noch schneller, leichter und intuitiver werden kann. Das eindeutige Ziel sind dabei die weitestgehend automatischen Systeme.

\subsubsection{Neue Untersuchungsmethoden}

Die moderne Computerspiel-Technik zeigt, wie intuitiv Systeme für den Anwender konzipiert sein können. Darüber hinaus können Bewegungen im Raum fast zeitgleich virtuell abgebildet werden. Dies gilt sowohl für elektronische, als auch für optoelektronische Anwendungen. Geräte, die vom Aufbau her diesen Systemen ähneln, werden schon heute für die Unterkieferbewegungsaufzeichnung eingesetzt.

Durch ihr minimales Equipment stellt die Videografie eine Besonderheit in diesem Forschungsbereich dar und macht sie besonders interessant. Derzeit werden für die Videoanalyse eine Kamera, ein Computer mit entsprechender Software und Marker benötigt.

Die Umsetzung dieser Analyseform könnte in naher Zukunft so aussehen, dass nur noch Marker und ein Smartphone mit entsprechenden Applications benötigt werden. Denn mit Hilfe einer automatisierten Marker-Trekking- und Bewegungsauswertungs-Application ließen sich Unterkieferbewegungen ganz einfach per Smartphone aufzeichnen und berechnen. Ein Analyseweg dieser Art wäre eine Möglichkeit, die Funktionsanalyse für jeden zugänglich zu machen und die Fortschritte der Technik für den Praxisgebrauch zu nutzen. 


\section{Zusammenfassung}

Die CMD ist eine in der Bevölkerung weit verbreitete Erkrankung mit hoher Behandlungsbedürftigkeit. Deren Ursachenermittlung hat eine fundamentale Relevanz für die Therapieplanung und Behandlung. Dafür sind heute elektronische Geräte zur Unterkieferfunktionsanalyse erhältlich. Da der Erwerb aber mit hohen Kosten verbunden ist, ist die Anschaffung nicht für alle klinischen Einrichtungen möglich. Darüber hinaus ist die Anbringung oft zeitaufwendig und die Anwendungen der Geräte und Softwareprogramme bedarf einer Einführung und Einarbeitung.

Aus diesem Grund sollte eine kostengünstige Alternativmethode entwickelt werden, die sowohl einfach in der Handhabung als auch mit einem geringen Gewicht und wenig Zeitaufwand verbunden ist. Außerdem sollten die Messungen mit auf dem Markt etablierten Geräten vergleichbare Ergebnisse liefern.

Um diese Anforderungen umzusetzen, wurde in der vorliegenden Untersuchung eine neuartige Technik von Dr. A. Braidot et al. auf die Untersuchungen der Unterkieferbewegungen angepasst.

Für die Analyse wurden lichtreflektierende Marker auf der Haut befestigt, die Bewegungen mit einer Videokamera aufgezeichnet und die Bewegungsbahnen mit Hilfe einer Computersoftware berechnet.

Um sowohl die Messergebnisse als auch den zeitlichen und finanziellen Aufwand der neuen Methode beurteilen zu können, wurde diese Videoanalyse mit einem auf dem Markt etablierten Funktionsanalysegerät (ARCUSßdigma, KaVo) verglichen.

In der durchgeführten Untersuchung wurde überprüft, ob die Ergebnisse beider Methoden vergleichbar sind (1), die unterschiedliche Lokalisation der Messpunkte (intraoral und extraoral) zu divergierenden Messergebnissen führt (2) und ob die Maxima-Werte der Bewegungen Aufschluss über eine Erkrankung des stomatognathen Systems geben (3).

Die Ergebnisse der Untersuchung zeigen, dass die Maxima-Werte der beiden Methoden nicht übereinstimmen (zu 1), was auf die unterschiedliche Lokalisation der Messpunkte zurückzuführen ist ( $z u$ 2). Aus diesem Grund sind die Bewegungsmaxima nicht für die konkrete Diagnose einer CMD geeignet (zu 3). 
Zusammenfassend lässt sich für die in dieser Studie durchgeführte Videoanalyse sagen, dass sie in ihrer derzeitigen Form noch einige Schwierigkeiten mit sich bringt, die aber künftig durch die Weiterentwicklung der Programme und einen optimierten Versuchsaufbau behoben werden können.

Eine Weiterführung der Untersuchungen ist somit wünschenswert, da durch die aufgeführten Veränderungen mit wenig Aufwand gute und schnelle Ergebnisse erzielt werden können, die darüber hinaus auch einen wichtigen Beitrag für die CMD-Forschung leisten. 


\section{Literaturverzeichnis}

Ahlers $\mathrm{M}$ O: Restaurative Zahnheilkunde mit dem Artex-System. 2. Auflage; dentaConcept, Hamburg 1998

Ahlers M O, Freesmeyer W B, Göz G, Jakstat H A, Koeck B, Meyer G, Ottl P, Reiber T, Seeher W D (2003a): Klinische Funktionsanalyse - Gemeinsame Stellungnahme der DGZMK und der Deutschen Gesellschaft für Funktionsdiagnostik und -therapie (FDT) in der DGZMK. D Zahnärztl Z $\underline{58}$ (7), 383-384

Ahlers M O, Freesmeyer W B, Göz G, Jakstat H A, Koeck B, Meyer G, Ottl P, Reiber T, Seeher W D (2003b): Instrumentelle, bildgebende und konsilliarische Verfahren zur CMD-Diagnostik. D Zahnärztl Z $\underline{58}$ (7), 385-386

Ahlgren J (1967): Mechanism of mastication. A quantitative cinematographic and electromyographic study of masticatory movements in children, with special reference to occlusion of the teeth. Am J Orthod $\underline{53}$ (3), 225-228

Al-Jundi M A, John M T, Setz J M, Szentpétery A, Kuss O (2008): Meta-analysis of Treatment Need for Temporomandibular Disorders in Adult Nonpatients. J Orofac Pain $\underline{22}(2), 97-107$

Al-Saleh M A, Armijo-Olivo S, Floress-Mir C, Thie N M (2012): Electromyography in diagnosing temporomandibular disorders. J Am Dent Assoc 143 (4), 351-362

Arnholz C R: Dreidimensionale Ultraschallmessung in gesunden und funktionsgestörten Kiefergelenken mit einem neu entwickelten Registrierbehelf, eine Nachuntersuchung und vergleichende Darstellung zur mechanischen Achsiographie. Med. Diss. Bonn 1994

Atkinson H F, Shepherd R W (1955): A preliminary report of investigations into mandibular movement. Aust Dent J $\underline{59}, 267-270$ 
Baeyens J P, Gilomen H, Erdmann B, Clijsen R, Cabri J, Vissers D (2013): In vivo measurement of the 3D kinematics of the temporomandibular joint using miniaturized electromagnetic trackers: technical report. Med Biol Eng Comput $\underline{51}$ (4), 479-484

Bellach B M, Knopf H, Thefeld W (1999): Der Bundes-Gesundheitssurvey 1998. Das Gesundheitswesen $\underline{60}$ (2), 59 - 68

Bernhardt O, Schwahn B (1999): Therapie mit Aufbißschienen. ZMK -ㅡ, 432-442

Bernhardt O, Schwahn B, Meyer G (1999): Craniomandibular disorders - comparative investigations with clinical examination and electronic axiography. Anat Anz 181 (1), 5153

Bewersdorff H J (1970): Elektrognathographie - elektronische dreidimensionale Messung und Registrierung von Kieferbewegungen Teil 3 - Studien über den Einfluss verschiedenartiger Faktoren auf dem Kauverlauf. Eur J Oral Sci $\underline{78}$ (1-4), 178-204

Beyron H (1964): Occlusal Relations and Mastication in Australian Aborigines. Acta Odontol Scand $\underline{22}, 597-678$

Braidot A, Brusa M H, Lestussi F E, Parera G P (2007): Biomechanics of Front and Back Squat exercises. J Phys, Conference Series $\underline{90} 012009$

Brewer A A, Hudson D C (1961): Application of miniaturized electronic devices to the study of tooth contact in complete dentures. A progress report. J Prosthet Dent 11 (1), 62-72

Bumann A (2007): Instrumentelle Funktionsanalyse - Das innovative EAEF-Modul des neuen KaVo ARCUSdigma II. Das deutsche Zahnärzteblatt 116 (3), 123

Buschang P H, Throckmorton G S, Travers K H, Hayasaki H (2001), Incisor and mandibular condylar movements of young adult femals during maximum protrusion and 
laterotrusion of the jaw. Arch Oral Biol $\underline{46}, 39-48$

Carlsson G E (1999): Epidemiology and treatment need for temporomandibular disorders. J Orofac Pain 13(4), 232-237

Casanova-Rosado J F, Medina-Solis C E, Casanova-Rosado A, Vallejos-Sanchez A A, Patiño-Marin N, Maupome G, Gomez-Gomez V (2012): Clinical characterization of mouth opening among Mexican adolescents and young adults. J Dent Sci $\underline{7}, 81-84$

Costa M D, Rocha Torres Froes G, Santos C N (2012): Evaluation of occlusal factors in patients with temporomandibular joint disorder. Dental Press J Orthod 17 (6), 61-68

Detamore M S, Alhanasiou K A (2003): Structure and Funktion of the Temporomandibular Joint Disc: Implications for Tissue Engineering. J Oral Maxillofac Surg $\underline{61}$ (4), 494-506

DosSantos M F, Martikainen I K, Nascimento T D, Love T M, Deboer M D, Maslowski E C, Monteiro A A, Vincent M B, Zubieta J K, DaSilva A F (2012): Reduced basal ganglia $\mu$-opioid receptor availability in trigeminal neuropathic pain: a pilot study. Mol Pain $\underline{8}$ (74), 1-6

Dworkin S F (2010): Research Diagnostic Criteria for Temporomandibular Disorders: current status and future relevance. J Oral Rehabil $\underline{37}, 734-743$

Ellerbrock H (2009): Digitale Bewegungsaufzeichnung. Digital Dent News, 3. Jahrg., 6-13

Engelhardt J P, Hugger A, Jakstat H A, Kordaß B (2002): Instrumentelle Funktionsanalyse. D Zahnärztl Z $\underline{57}$ (12), 659

Engelskirchen C: Untersuchung zur elektronischen Vermessung der transversalen und sagittalen Unterkieferbewegung. Med. Diss. Bonn 1998 
Fang J J, Kuo T H (2008): Modelling of mandibular movement. Comput Biol Med $\underline{38}$, 11521162

Furtado D A, Pereira A A, Andrade A, Bellomo D P, Silva M R (2013): A specialized motion capture system for real-time analysis of mandibular movements using infrared cameras. BioMedical Eng OnLine 12 (17), 1-16

Gillings B R D (1967): Photoelectric mandibulography: A technique for studying jaw movements. J Prosthet Dent 17 (2), 109-121

Glickman I, Pameijer J H N, Roeber F W (1968): Intraoral occlusal telemetry part I. A multifrequency transmitter for registering tooth contacts in occlusion. J Prosthet Dent 19 (1), 60-68

Glickman I, Pameijer J H N, Roeber F W, Brion M A (1969): Functional occlusion as revealed by miniaturized radio transmitters. Dent Clin North Am 13 (3), 667-679

Gonçalves D A, Dal Fabbro A L, Campos J A, Bigal M E, Speciali J G (2010): Symptoms of temporomandibular disorder in the population: an epidemiological study. J Orofac Pain $\underline{24}(3), 270-278$

Goodson J M, Johansen E (1975): Analysis of human mandibular movement. Monogr Oral Sci $\underline{5}, 1-80$

Graf H, Zander H A (1963): Tooth contact patterns in mastication. J Prosthet Dent 13 (6), 1055-1066

Hamborg R, Karlsson S (1996): Movement and signal analysis by means of a computerassisted system. J Oral Rehabil $\underline{23}$ (2), 121-128

Hanel G: Funktionsphysiologisch orientierte Praxis: Ein Leitfaden für den allgemeinen 
Praktiker. Quintessenz Bibliothek. Quintessenz Verlags-GmbH, Berlin 1984

Hildebrand G Y: Studies in the masticatory movements of the human lower jaw, Band 61; Skandinavisches Archiv für Physiologie, Berlin 1931

Hobo S, Mochizuki S (1983): A kinematic investigation of mandibular border movement by means of an electronic measuring system. Part I. Development of the measuring system. J Prosthet Dent $\underline{50}$ (3), 368-373

Ingawalé S M, Goswami T: Biomechanics of the Temporomandibular Joint. In: Human Musculoskeletal Biomechanics, hrsg. v. Goswami T, InTech, Rijeka, 2012, 159-182

Jankelson B, Hoffman G M, Hendron J A (1952): The physiology of the stomatognathic system. J Am Dent Assoc $\underline{46}$ (4), 375-386

Jemt T, Karlsson S (1980): Mandibular movements during mastication before and after rehabilitations with new complete dentures recorded by light-emitting-diodes. Swed Dent J $\underline{4}$ (5), 195-200

Jemt T, Hedegård B (1982): The relative movements of the chin and the mandible during chewing. J Oral Rehabil $\underline{9}$ (3), 253-258

Karlsson S (1977): Recording of mandibular movements by intraorally placed light emitting diodes. Acta Odontol Scand $\underline{35}$ (2), 111-117

Kjellberg H, Kliaridis S, Karlsson S (1995): Characteristics of masticatory movements and velocity in children with juvenile chronic arthritis. J Orofac Pain $\underline{9}$ (1), 64-72

Knap F J, Richardson B L, Bogstad J (1970a): Motions of the mandible related to modern gnathologic concepts. J Prosthet Dent $\underline{24}$ (2), 148-158 
Knap F J, Richardson B L, Bogstad J (1970b): Study of the mandible motion in six degrees of freedom. J Dent Res 49 (2), 289-292

Kobs G, Diziulyte A, Kirlys R, Stacevicius M (2007): Reliability of ARCUSdigma (KaVo®) in diagnosing temporomandibular joint pathology. Stomatologiia (Mosk) $\underline{9}$ (2), 47-55

KohImann T (2002): Epidemiologie orofazialer Schmerzen. Der Schmerz 16 (5), 339-345

Körber E, Luckenbach A (1981): Dreidimensionale Darstellung der Bewegung einzelner Punkte eines Kiefermodells im Artikulator. D Zahnärztl Z 36, 462-466

Koseki M, Niitsuma A, Inou N, Maki K, Three-dimensional Display System of Individual Mandibular Movement, in: Complex Medical Engineering, hrsg. v. Wu J L, Ito K u.a.; Springer Verlags-GmbH, Tokyo, 2007, 117-127

Kralijevic S, Panduric J, Badel T, Dulcic N (2003): Registration and Measurement of Opening and Closing Jaw Movements and Rotational Mandibular Capacity by Using the Method of Electronic Axiography. Coll Antropol 27, 51-59

Krestan C, Lomoschitz F, Puig S, Robinson S (2001): Internal derangement of the temporomandibular joint. Radiologe $\underline{41}(9), 741-747$

Krogh-Poulsen W: Die klinische Untersuchung und Befundaufnahme am Kiefergelenkpatienten durch den Zahnarzt; in: Okklusion und Kiefergelenk, hrsg. v. Gerber A und Weber F; Zahnärztliches Instistut der Universität Zürich, Zürich 1973, 110118

Lehmann K M, Hellwig E, Wenz H J: Zahnärztliche Propädeutik. Einführung in die Zahnheilkunde. 11. Auflage; Deutscher Zahnärzte Verlag, Köln 2009

LeResche L (1997): Epidemiology of temporomandibular disorders: implications for the 
investigation of etiologic factors. Crit Rev Oral Biol Med 8 (3), 291-305

Lobbezoo F, Drangsholt M, Peck C, Sato H, Kopp S, Svensson P (2004): Topical Review: New Insights into the Pathology and Diagnosis of Disorders of the Temporomandibular Joint. J Orofac Pain 18 (3), 181-191

Lochmiller W, Bumann A, Groot Landeweer G (1991): Zur Wertigkeit der elektronischen Axiographie in der klinischen Funktionsdiagnostik. J Orofac Orthop $\underline{52}$ (5), 268-273

Luce C E (1889): The movement of the lower jaw. Boston Med Surg J 121, 8-11

Manfredini D, Piccotti F, Ferronato G, Guarda-Nadini L (2010): Age peaks of different $\mathrm{RDC} / \mathrm{TMD}$ diagnoses in a patient population. J Dent $\underline{38}, 392-399$

Manfredini D, Guarda-Nadini L, Winocur E, Piccotti F, Ahlberg J, Lobbezoo F (2011): Research diagnostic criteria for temporomandibular disorders: a systematic review of axis I epidemiologic findings. Oral Surg Oral Med Oral Pathol Oral Radiol Endod 112 (4), 453-462

McNeill C (1997): History and evolution of TMD concepts. Oral Surg Oral Med Oral Pathol Oral Radiol Endod $\underline{83}$ (1), 51-60

Mehulic K, Gospic R K, Dundjer A, Skrinjaric T, Stefanicic S, Vojvodic D, Perinic M (2009): Optoelectronic Pantography Diagnostics of Temporomandibular Disorders in Patients with Bruxism. Coll Antropol 33 (3), 849-856

Mesqui F, Palla S (1985): Real-time non-invasive recording and display of functual jaw movements. J Oral Rehabil $12,541-547$

Mumford J M: Kiefer- Gesichtsschmerz. Deutscher Ärzte-Verlag, Köln 1989 
Munzesheimer F (1928): Photografische Registrierung der Kieferbewegungen und ihre Auswertung. D Zahnärztl Wochenschr 10, 425-449

Neill D J (1967): Studies of tooth contact in complete dentures. Br Dent J 123, 369-378

Okeson J P, De Leeuw R (2011): Differential Diagnosis of Temporomandibular Disorders and Other Orofacial Pain Disorders. Dent Clin North Am $\underline{55}$ (1), 105-120

Pinheiro A P, Pereira A A, Andrade A O, Bellomo D (2011): Measurement of jaw motion: the proposal of a simple and accurate method. J Med Eng Technol $\underline{35}$ (3-4), 125-126

Platzer W: Taschenatlas Anatomie - 1 Bewegungsapparat. 10. Auflage; Thieme, Stuttgart 2009

Pröschel P (1987a): Zur Problematik der Interpretation von funktionellen Unterkieferbewegungen. D Zahnärztl Z 42, 696-700

Pröschel P (1987b): An extensive classification of chewing patterns in the frontal plane. J Craniomand Pract $\underline{5}(1), 55-63$

Rasch B, Friese M, Hoffmann W J, Naumann E: Quantitative Methoden 1. Einführung in die Statistik für Psychologen und Sozialwissenschaftler, 3. Auflage; Springer Verlag, Berlin-Heidelberg 2010

Röhrle O, Waddell J N, Foster K D, Saini H, Pullan A J (2009): Using a Motion-Captur System to Record Dynamic Articulation for Application in CAD/CAM Software. J Prosthodont 18 (8), 703-710

Sadat-Khonsari R, Fenske C, Kahl-Nieke B, Kirsch I, Jüde H D (2003): Momentane Drehzentren des Unterkiefers bei Patienten mit und ohne Funktionsstörung des Kiefergelenks. J Orofac Orthop $\underline{64}, 256-264$ 
Schierz O, Reißmann D R (2008): Die elektronische Vermessung der Gelenkbahn. Digital Dent News 2, 21-27

Schierz O, Klinger N, Schön G, Reißmann D R (2014): The Reliability of Computerized Condylar Path Angle Assessment. Int J Comput Dent 17 (1), 35-51

Schmitter M, Giannakopoulos N N, Eberhard L, Hellmann D, Kelter M, Schindler H (2011): Elektronische Messverfahren. Quintessenz Zahntech 37 (9), 1212-1220

Schulte W (1970): Zur funktionellen Behandlung der Myoarthropathien des Kauorgans: Ein diagnostisches und physiotherapeutisches Programm. D Zahnärztl Z $2 \underline{5}$ (3), 422-436

Shepherd R W (1960): A further report on mandibular movement. Aust Dent J $\underline{5}$ (6), 337342

Siebert G K: Zahnärztliche Funktionsdiagnostik - mit und ohne Hilfsmittel. 2.Auflage; Carl Hanser Verlag, München-Wien 1987

Simonet P F (1981): Influence of TMJ dysfunction on Bennett movement as recorded by a modified Pantograph. Part I: Literature review. J Prosthet Dent $\underline{46}$ (4), 437-442

Sinfomed: SinfoMed K7 - Myozentrische Bissregistrierung. URL: http://www.sinfomed.de/zahnmedizin/k7/ (14.09.2014)

Soboleva U, Laurina L, Slaidina A (2005 a): Jaw tracking device - historical review of methods development. Part I. Stomatologija (Mosk) $\underline{7}, 67-71$

Soboleva U, Laurina L, Slaidina A (2005 b), Jaw tracking device - historical review of methods development. Part II. Stomatologija (Mosk) $\underline{7}, 72-76$

Strub J R, Kern M, Türp J C, Witkowski S, Heydecke G, Wolfart S: Curriculum Prothetik 
Band 1. 4. Auflage; Quintessenz Verlags-GmbH, Berlin 2011

Szentpétery A (1993): Clinical Utility of Mandibular Movement Ranges. J Orofac Pain $\underline{7}$ (2), 163-168

Thouren G (1914): Articulatio mandibularis och underkakens rorelser i „sagittal“ och „horisontellt“ plan. Sven Tandlak Tidskr $\underline{2}, 32-48$

Travers K H, Buschang P H, Hayasaki H, Throckmorton G S (2000): Associations between incisor and mandibular condylar movements during maximum mouth opening in humans. Arch Oral Biol $\underline{45}, 267-275$

Türp J C (2003): Ist die instrumentelle Funktionsanalyse eine empfehlenswerte Maßnahme zur Diagnostik kraniomandibulärer Funktionsstörungen?. D Zahnärztl Z $\underline{58}$, 477-481

Türp J C, Schindler H J, Bartzela T (2005): Schmerzhafte Myoarthropathien des Kausystems - evidenzbasierte Diagnostik. Kieferorthop 19, 173-181

Wegmann U (2013): Wie viel Okklusion braucht der Mensch?. Zahntech Mag 17 (5), 284296

Woelfel J B, Hickey J C, Allison M L (1962): Effect of posterior tooth form on jawand denture movement. J Prosthet Dent 12 (5), 922-939

Yoon H J, Zhao K D, Rebellato J, An K N, Keller E E (2006): Kinematic study of the mandible using an electromagnetic tracking device and custom dental appliance: Introducing a new technique. J Biomech $\underline{39}, 2325-2330$

Yurkstas A A, Emerson W H (1954): A study of tooth contact during mastication with artificial dentures. J Prosthet Dent $\underline{4}(2), 168-174$ 
Zubieta J K, Heitzeg M M, Smith Y R, Bueller J A, Xu K, Xu Y, Koeppe R A, Stohler C S, Goldman D (2003): COMT val158met genotyp affects mu-opioid neurotransmitter responses to a pain stressor. Science 299 (5610), 1240-1243 


\section{Abbildungsverzeichnis}

Abb. 1: RDC/CMD Achse I und II - Patientenbeispiel A und B....................................19

Abb. 2: Proband mit angelegtem Gesichtsbogen und aufgeklebten Markern.....................35

Abb. 3: ARCUS $₫$ digma mit mobilem Monitor................................................................. 37

Abb. 4: Darstellung der Bewegungsbahnen im ARCUS®digma-Softwareprogramm.......37

Abb. 5: Bewegungsgraphen und Liste der Maxima-Werte............................................. 37

Abb. 6: Dreidimensionale Ansicht der Unterkieferbewegungen.......................................

Abb. 7: Darstellung der verschiedenen Marker................................................................39

Abb. 8: Grenzbewegungen in der Frontalebene...........................................................

Abb. 9: Grenzbewegungen in der Sagittalebene („Posselt sagittal")...............................41

Abb. 10: Bewegungsablauf der frontalen Grenzbewegung......................................... 41

Abb. 11: Bewegungsablauf der sagittalen Grenzbewegung......................................... 41

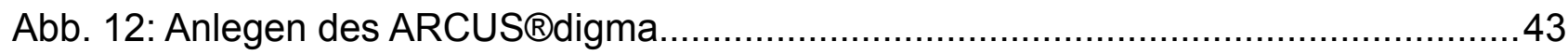

Abb. 13: Kameraaufnahme von frontal mit angebrachten Markern.................................44

Abb. 14: Kameraaufnahme von sagittal mit angebrachten Markern................................44

Abb. 15: Frontale Aufnahme mit Skala (C1-4) und Markierungen (AD1 und AD2).............47

Abb. 16: Grafische Darstellung des Ablaufs der Datentransformation............................... 48

Abb. 17: Maxima-Werte für die frontale Grenzbewegung............................................49

Abb. 18: Maxima-Werte für die sagittale Grenzbewegung............................................ 49

Abb. 19: Frontale Grenzbewegung ausgehend vom Kinnpunkt.......................................50

Abb. 20: Sagittale Grenzbewegung ausgehend vom Kinnpunkt. ....................................51

Abb. 21: Frontale Grenzbewegung ausgehend vom Inzisalpunkt...................................52

Abb. 22: Sagittale Grenzbewegung ausgehend vom Inzisalpunkt.................................52

Abb. 23: Grafische Darstellung der Vergleichsberechnung (Messpunktabstand $2 \mathrm{~cm}$ )......54 
Abb. 24: Grafische Darstellung der Vergleichsberechnung (Messpunktabstand $3 \mathrm{~cm}$ ).....54

Abb. 25: Grafische Darstellung der möglichen Bewegungsabweichungen.......................55

Abb. 26: Laterotrusion links: Darstellung der intraindividuellen Mediane.........................58

Abb. 27: Laterotrusion links: Darstellung der verbundenen intraindividuellen Mediane.....59

Abb. 28: Laterotrusion rechts: Darstellung der intraindividuellen Mediane......................59

Abb. 29: Laterotrusion rechts: Darstellung der verbundenen intraindividuellen Mediane...59

Abb. 30: Mundöffnung frontal: Darstellung der intraindividuellen Mediane.....................60

Abb. 31: Mundöffnung frontal: Darstellung der verbundenen intraindividuellen Mediane...60

Abb. 32: Mundöffnung sagittal: Darstellung der intraindividuellen Mediane.....................60

Abb. 33: Mundöffnung sagittal: Darstellung der verbundenen intraindividuellen Mediane. 61

Abb. 34: Protrusion: Darstellung der intraindividuellen Mediane.................................61

Abb. 35: Protrusion: Darstellung der verbundenen intraindividuellen Mediane...............61

Abb. 36: Laterotrusion links: Intraindividuelle Differenzmittelwerte..............................63

Abb. 37: Laterotrusion rechts: Intraindividuelle Differenzmittelwerte...........................63

Abb. 38: Mundöffnung-frontal: Intraindividuelle Differenzmittelwerte.........................63

Abb. 39: Mundöffnung-sagittal: Intraindividuelle Differenzmittelwerte..........................64

Abb. 40: Protrusion: Intraindividuelle Differenzmittelwerte .................................... 64

Abb. 41: Laterotrusion links: Intraindividuelle Mediane mit Faktorenrechnung.................65

Abb. 42: Laterotrusion rechts: Intraindividuelle Mediane mit Faktorenrechnung..............65

Abb. 43: Mundöffnung sagittal: Intraindividuelle Mediane mit Faktorenrechnung.............65

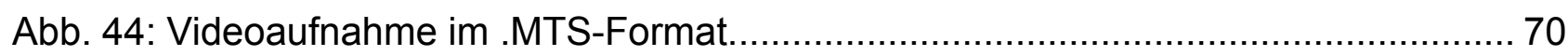

Abb. 45: Videoaufnahme nach dem Konvertieren ins .AVI-Format.............................. 70

Abb. 46: Ausrichtung des Unterkieferbogens zur Kalibrierungsebene.........................72

Abb. 47: Ausrichtung des Kinnmarkers zur Kalibrierungsebene..............................72

Abb. 48: Rotation des Unterkiefers bei der Laterotrusionsbewegung ........................... 80 


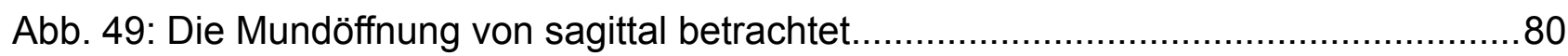

Abb. 50: Projektion der Mundöffnung auf die Frontalebene...................................... 81

Abb. 51: Grafische Darstellung der Vergleichsberechnung (Messpunktabstand $2 \mathrm{~cm}$ ).....83

Abb. 52: Grafische Darstellung der Vergleichsberechnung (Messpunktabstand $3 \mathrm{~cm}$ ).....83 


\section{Tabellenverzeichnis}

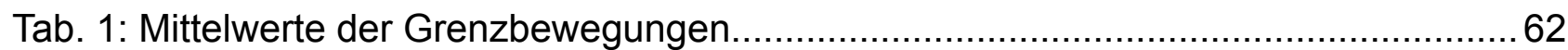

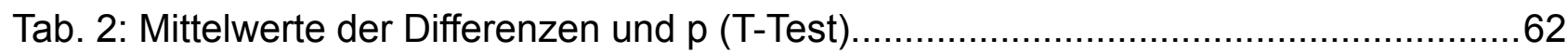

Tab. 3: Die prozentuale Übereinstimmung für die frontalen Bewegungsabschnitte...........66

Tab. 4: Die prozentuale Übereinstimmung für die sagittalen Bewegungsabschnitte.........66

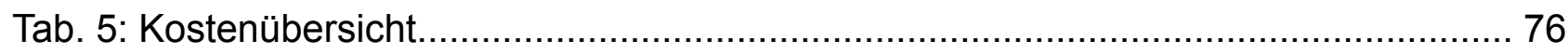

Tab. 6: Mittelwerte der einzelnen Bewegungen....................................................... 77

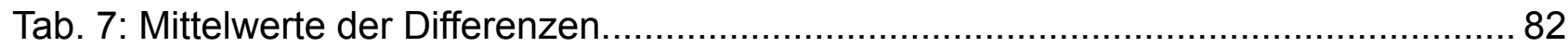




\section{Anhang}

\subsection{Ethikvotum}

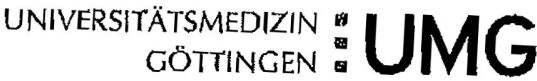

Eethukommission der Med. Fakuust, Von-Slebolds Strabe 3, 37075 Gettingen

\section{Herm}

PD Dr, med. Nikolaus Gersdorff

Poliklinik für Prothetik

Robert-Koch-Straße 40
Medizinische Fakultał Ethikkommission Vorsitzender: Prof. Dr, Jurgen Brockmöller Referentin Regierungsratin Doris Wettschereck $0551 / 39-8644$ Telefon

Von-Siebold-Straße 3, 37075 Gottingen Adresee $0 \$ 51 / 38-6629$ Telefon $0561 / 39-9536 \mathrm{Fax}$ ethik@med.uni-goettingen.de E.Mail www.ethikkommission.med.uni-goettingen.de 17.07.2013 br - fr - gö Datum

vorab per Fax: 22800 6/5/13 (bltte stets angeban)

Antragsnummer: Vergleichende Untersuchungen zur Genauigkeit von elektronischer Funktionsanalyse Studientitel: und Videosequenz-Analyse

Antragsteljer: PD Dr. med. Nikolaus Gersdorff, Poliklinik fur Prothetik, UMG Doktorandin: Janna Marie Edelhoff

Folgende Unterlagen wurden zur Bewertung nachgerejcht:

- Anschreiben mit Stellungnahmen vom 11.07.2013

- Oberarbejtetes Studienprotokoll

- Uberarbeitete Einwilligungserklärung

- Überarbeltete Probandeninformation

Sehr geehrter Herr Dr. Gersdorff, sehr geehrte Damen und Herren,

nach Ergänzung der o.g. Dokumente und Beantwortung der im vorläufigen Votum aufgefühten Fragen in ihrem Schreiben vom 11.07.2013 bestehen nunmehr keine ethischen und rechtlichen Bedenken gegen die Durchführung des oben genannten Forschungsvorhabens.

Wir wünschen Ihnen viel Erfolg bei der Durchfuhrung ithres Projektes.

Unabhanglg vom Beratungsergebnis macht die Ethik-Kommission darauf aufmerksam, dess die ethisch $\Theta$ und rechtliche Verantwortung für die Durchführung einer wiesenschaftlichen Studie beim verantwortichen Studienarzi und aller an der Btudie beteillgten Ärzte liegt

Alle Anderungen im Studienprotokoll müssen der Ethik-Kommission vorgelegt werden und düren erst nach der zustimmenden Bowertung umgesetzt werden.

Uber alle schwerwiegenden unerwarteten unerwünschten Erelgnisse, die während der Studie auftreten und die SIcherheit der Studienteilnehmer oder die Durchfuhrung der Studie beeinträchtigen Könnten, muss die Ethik-Kommission unterrichtet werden.

Der Abschluss/Abbruch der Studie ist mitzuteilen und eln Abschlußbericht vorzuiegen.

Auf die Einhaltung einschlëgiger Gesetze und Rechtsvorschriften wird hingewiesen. Die nach Rechtslage notwendigen Unterrichtungen (u. A. Anderung des Studienprotokolls, Meldung von Zwischenfällen, neus Datenlage, Nachmeldung von Prüfentren, Abschlussbericht) sind der Ethlk-Kommission unverzugiflch vorzulegen.

Die Ethik-Kommission bestátigt, dass sie auf Grundlage nationaler Gesetze, Vorschriften sowie der GCP/CH-Richtlinie arbeiket.

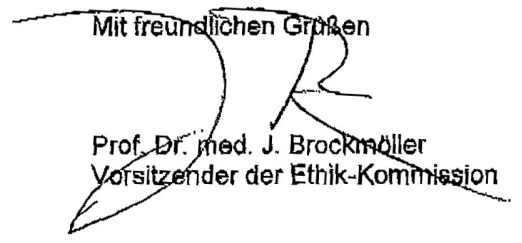




\subsection{Patientenaufklärung}

Sehr geehrte(r) Patient(in)!

Wir bitten um die freiwillige Teilnahme an dieser Studie. Sie können jederzeit die Teilnahme widerrufen, ohne Angabe von Gründen und ohne Nachteile erwarten zu müssen. Nach Ihrem Widerruf erfolgt unverzüglich die Vernichtung Ihrer personenbezogenen Daten.

\section{Hintergrund}

Eine Funktionsstörung des Kauorgans äußert sich bei jedem Patienten individuell. Es können unter Anderem schmerzhaftes Kiefergelenkknacken, eine eingeschränkte Mundöffnung, Asymmetrien während der Mundöffnung und Muskelverspannungen auftreten. In diesen Fällen ist das Kiefergelenk mit seinen angrenzenden Strukturen betroffen.

Um sich ein genaues Bild der Störung zu machen, können verschiedene Verfahren genutzt werden. In unserer Studie sollen zwei dieser Untersuchungsmethoden angewandt werden, um die Ursachen der Funktionsstörung bzw. Schmerzen zu ermitteln.

\section{Untersuchungen}

\section{ARCUS®digma - elektronischer Gesichtsbogen}

Die Bewegung des Unterkiefers wird mit Hilfe eines elektronischen Gesichtsbogens digital aufgezeichnet. Dabei werden die Neigung der Gelenkbahn und die Grenzbewegungen des Unterkiefers registriert. Auf dem Bildschirm sehen Patient und Behandler zum einen die Gelenkbahnen in Form von Graphen, zum anderen zeigt eine 3D- Aufnahme die Bewegung der knöchernen Anteile zueinander.

\section{Video-Aufnahmen}

Die Unterkieferbewegungen werden mit Hilfe von Videoaufnahmen aufgezeichnet und Störungen anhand dieser ermittelt.

Als Ergebnis werden reproduzierbare Befunde und Werte für die Kiefergelenkbewegungen gewonnen. 


\section{Ziel}

Die Achsiogramme sollen erstellt werden, um Ihre Kiefergelenkbewegungen mit Hilfe von zwei verschiedenen Methoden aufzuzeichnen. Die Daten werden dann miteinander verglichen, um ein möglichst genaues Ergebnis der Ursachen von eventuellen Beschwerden zu erhalten.

\section{Datenschutz}

Ihre personenbezogenen Daten werden erhoben und gespeichert und unterliegen den Regelungen des Datenschutzgesetzes. Die Datenerhebung erfolgt anonym, wird mittels einer Codenummer verschlüsselt und so gespeichert, dass Dritten eine $\mathrm{Zu}$ ordnung der Untersuchungsergebnisse zu Ihrer Person nicht möglich ist. Die Aufbewahrung erfolgt in verschließbaren Räumlichkeiten, zu denen nur die klinischen Prüfer Zugang haben. Die Speicherungszeit dieser Daten umfasst die Studiendauer. Eine Weitergabe erfolgt nicht. Die Daten werden bei einem Widerruf der Einverständniserklärung während der Teilnahme an dieser Studie unwiederbringlich vernichtet. Außerdem werden die codierten Daten nur zu Untersuchungszwecken verwendet und nach der Auswertung vernichtet. Eine abschließende Veröffentlichung der Ergebnisse erfolgt nur in anonymisierter Form.

Es entstehen durch die Untersuchung keine zusätzlichen Kosten für Sie. Insgesamt dauert die Untersuchung ungefähr 45 Minuten.

Für Rückfragen stehen Ihnen der Studienleiter und der durchführende Zahnarzt der Abteilung Prothetik unter den oben aufgeführten Telefonnummern gern zur Verfügung.

Vielen Dank für Ihre Teilnahme. 


\subsection{Einverständniserklärung}

Ich, wurde von meinem Arzt vollständig über Wesen, Bedeutung und Tragweite der klinischen Untersuchung mit dem Titel „Vergleichende Untersuchungen zur Genauigkeit von elektronischer Funktionsanalyse und Videosequenz-Analyse" aufgeklärt.

Ich habe den Aufklärungstext gelesen und verstanden. Ich hatte die Möglichkeit, Fragen zu stellen, und habe die Antworten verstanden und akzeptiere diese. Mein Arzt hat mich über die mit der Teilnahme an der Studie verbundenen Risiken und den möglichen Nutzen informiert.

Die Verwendung Ihrer personenbezogenen Daten erfolgt unter datenschutzrechtlichen Bestimmungen. Ihr Einverständnis zur Speicherung und Auswertung dieser Daten unterliegt diesen Bedingungen.

Mir ist bekannt, dass ich jederzeit und ohne Angaben von Gründen diese Zustimmung widerrufen kann, ohne dass sich dieser Entschluss nachteilig auswirkt. Ich habe eine Kopie der Patienteninformation und dieser Einwilligungserklärung erhalten.

Ich wurde informiert, dass im Rahmen dieses Forschungsvorhabens personenbezogene Daten erhoben und in pseudonymisierter (verschlüsselter) Form aufgezeichnet und gespeichert werden. Die Datenspeicherzeit beträgt 10 Jahre. Die personenbezogenen Daten werden nicht an Dritte weitergegeben. Ich weiß, dass ich mein Einverständnis zur Speicherung der personenbezogenen Daten jederzeit widerrufen kann. Im Falle des Widerrufs werden alle personenbezogenen Daten gelöscht.

Ich erkläre hiermit meine freiwillige Teilnahme an dieser Studie.

Ort und Datum

Unterschrift des Teilnehmers

Ort und Datum

Unterschrift des Prüfarztes 


\subsection{Bewertungskriterientabelle}

\begin{tabular}{|c|c|c|c|c|c|c|c|c|c|c|c|c|}
\hline PF & & Code $A$ & AD1 & Video1 & AD2 & Video2 & AD3 & Video3 & AD4 & Video4 & AD5 & Video5 \\
\hline \multirow{3}{*}{ Laterotrusion left } & konkav & 1 & & & & & & & & & & \\
\hline & konvex & 2 & & & & & & & & & & \\
\hline & gerade & 3 & & & & & & & & & & \\
\hline \multirow{2}{*}{ Left endpoint } & spitz & 1 & & & & & & & & & & \\
\hline & stumpf & 2 & & & & & & & & & & \\
\hline \multirow{3}{*}{ Left opening } & konkav & 1 & & & & & & & & & & \\
\hline & konvex & 2 & & & & & & & & & & \\
\hline & gerade & 3 & & & & & & & & & & \\
\hline \multirow{2}{*}{ Left opening endpoint } & spitz & 1 & & & & & & & & & & \\
\hline & stumpf & 2 & & & & & & & & & & \\
\hline \multirow{7}{*}{ Left closing } & Deflexion rechts & 1 & & & & & & & & & & \\
\hline & Deflexion links & 2 & & & & & & & & & & \\
\hline & Deviation rechts & 3 & & & & & & & & & & \\
\hline & Deviation links & 4 & & & & & & & & & & \\
\hline & $\begin{array}{l}\text { Deflexion rechts } \\
\text { mit Deviation }\end{array}$ & 5 & & & & & & & & & & \\
\hline & $\begin{array}{l}\text { Deflexion links } \\
\text { Mit Deviation }\end{array}$ & 6 & & & & & & & & & & \\
\hline & gerade & 7 & & & & & & & & & & \\
\hline \multirow{3}{*}{ Laterotrusion right } & konkav & 1 & & & & & & & & & & \\
\hline & konvex & 2 & & & & & & & & & & \\
\hline & gerade & 3 & & & & & & & & & & \\
\hline \multirow{2}{*}{ Right endpoint } & spitz & 1 & & & & & & & & & & \\
\hline & stumpf & 2 & & & & & & & & & & \\
\hline \multirow{3}{*}{ Right opening } & konkav & 1 & & & & & & & & & & \\
\hline & konvex & 2 & & & & & & & & & & \\
\hline & gerade & 3 & & & & & & & & & & \\
\hline \multirow{2}{*}{ Right opening endpoint } & spitz & 1 & & & & & & & & & & \\
\hline & stumpf & 2 & & & & & & & & & & \\
\hline \multirow{7}{*}{ Right closing } & Deflexion rechts & 1 & & & & & & & & & & \\
\hline & Deflexion links & 2 & & & & & & & & & & \\
\hline & Deviation rechts & 3 & & & & & & & & & & \\
\hline & Deviation links & 4 & & & & & & & & & & \\
\hline & $\begin{array}{l}\text { Deflexion rechts } \\
\text { Mit Deviation }\end{array}$ & 5 & & & & & & & & & & \\
\hline & $\begin{array}{l}\text { Deflexion links } \\
\text { Mit Deviation }\end{array}$ & 6 & & & & & & & & & & \\
\hline & gerade & 7 & & & & & & & & & & \\
\hline \multirow{2}{*}{ Opening endpoints } & geschlossen & 1 & & & & & & & & & & \\
\hline & offen & 2 & & & & & & & & & & \\
\hline \multirow{2}{*}{ Closing endpoints } & geschlossen & 1 & & & & & & & & & & \\
\hline & offen & 2 & & & & & & & & & & \\
\hline \multicolumn{13}{|l|}{ PS } \\
\hline \multirow{3}{*}{ Protrusion } & konvex & 1 & & & & & & & & & & \\
\hline & konkav & 2 & & & & & & & & & & \\
\hline & gerade & 3 & & & & & & & & & & \\
\hline \multirow{2}{*}{ Protrusion endpoint } & spitz & 1 & & & & & & & & & & \\
\hline & stumpf & 2 & & & & & & & & & & \\
\hline & konvex & 1 & & & & & & & & & & \\
\hline Opening & konkav & 2 & & & & & & & & & & \\
\hline & gerade & 3 & & & & & & & & & & \\
\hline Opening endpoint & spitz & 1 & & & & & & & & & & \\
\hline & stumpf & 2 & & & & & & & & & & \\
\hline & konvex & 1 & & & & & & & & & & \\
\hline Closing & konkav & 2 & & & & & & & & & & \\
\hline & gerade & 3 & & & & & & & & & & \\
\hline Closing endnoint & geschlossen & 1 & & & & & & & & & & \\
\hline & offen & 2 & & & & & & & & & & \\
\hline
\end{tabular}




\section{Danksagung}

Mein besonderer Dank gilt Herrn PD Dr. med. dent. N. Gersdorff für die Annahme der Doktorvaterschaft, die Unterstützung und Betreuung, die Bereitstellung der Räumlichkeiten und die Möglichkeit, meine Untersuchungen frei durchführen zu können.

Danken möchte ich darüber hinaus Herrn Prof. Dr. med. Dr. med. dent. W. Engelke, der meinen Aufenthalt an der Fakultät für Bioingenieurwesen an der Universidad Nacional de Entre Rios (UNER) in Oro Verde, Paraná (Argentinien) durch die Kontaktvermittlung erst ermöglicht hat.

Auch möchte ich Dr. A. Braidot und M. Frisoli von der Fakultät für Bioingenieurwesen an der Universidad Nacional de Entre Rios (UNER) in Oro Verde, Paraná (Argentinien) für die Bereitstellung der Software, die Einführung in die Programme und einen wunderbaren Forschungsaufenthalt in Argentinien danken.

Des Weiteren bedanke ich mich beim Institut für Medizinische Statistik der Georg-AugustUniversität Göttingen für die Bereitstellung und Einführung in die Statistikprogramme. Hier möchte ich besonders die Mitarbeiter Dr. S. Schneider und X. Schulz erwähnen. 